\title{
The Hadronic Final State at HERA
}

\author{
Paul R. Newman* \\ School of Physics and Astronomy, University of Birmingham, Birmingham B15 2TT, UK \\ Matthew Wing \\ Department of Physics and Astronomy, University College London, Gower Street, London WC1E 6BT, UK; \\ DESY, Notkestrasse 85, 22607 Hamburg, Germany
}

(Dated: March 20, 2014)

\begin{abstract}
The hadronic final state in electron-proton collisions at HERA has provided a rich testing ground for development of the theory of the strong force, QCD. In this review, over 200 publications from the H1 and ZEUS Collaborations are summarised. Short distance physics, the measurement of processes at high energy scales, has provided rigorous tests of perturbative QCD and constrained the structure of the proton as well as allowing precise determinations of the strong coupling constant to be made. Non-perturbative or low energy processes have also been investigated and results on hadronisation interpreted together with those from other experiments. Searches for exotic QCD objects, such as pentaquarks, glueballs and instantons have been performed. The subject of diffraction has been re-invigorated through its precise measurement, such that it can now be described by perturbative QCD. After discussion of HERA, the H1 and ZEUS detectors and the techniques used to reconstruct differing hadronic final states, the above subject areas are elaborated. The major achievements are then condensed further in a final section summarising what has been learned.
\end{abstract}

\section{Contents}

\section{Introduction}

II. Reconstruction of Final States at HERA
A. The H1 and ZEUS Detectors
B. Charged Particles
C. Electrons, Photons and Muons
D. Heavy Flavour Identification
E. Hadronic Jets
F. Photoproduction and DIS and How to Tell Them Apart 6
G. Very Forward Taggers
H. Triggering

III. Hadronic Final States and Short Distance Physics 8

A. Perturbative QCD Theory of the Hadronic Final State 9

B. Comparisons with Data

1. Jet Production

2. Jet Substructure

3. Prompt Photon Production

4. Heavy Quark Production

C. Extracting Information From the Data

D. Pushing the Boundaries of Applicability

1. Higher Orders

2. New Low $x$ Phenomena

3. Virtual Photon Structure

4. The Underlying Event

5. Discussion

E. Event Shapes

IV. Non-Perturbative Aspects and Hadronisation

A. Charged Particle Distributions

B. Bose-Einstein Correlations

C. Charm Fragmentation

D. Inelastic $J / \psi$ Production

E. Exotic and Unusual QCD

*Electronic address: p.r.newman@bham.ac.uk

${ }^{\dagger}$ Electronic address: m.wing@ucl.ac.uk
1. Pentaquark States

2. Instanton Searches

3. Search for Glueball States

4. Deuteron and Anti-Deuteron Production

V. Exclusive and Semi-Inclusive Processes

A. Introduction

B. Exclusive Production of $1^{--}$States

1. Kinematics and Experimental Selection

2. General Characteristics of Vector Meson Production

3. Vector Meson Production in QCD

4. Deeply Virtual Compton Scattering and Generalised Parton Densities

5. Exclusive Processes in Dipole Models and Low $\boldsymbol{x}$ Saturation

C. Inclusive Diffractive Dissociation

1. Kinematics and Experimental Selection of Diffraction

2. Inclusive Diffraction Data

3. Factorisation Studies

4. Energy Dependences and Soft Phenomenology

5. QCD Phenomenology and Diffractive Parton Densities

6. Applications of Diffractive Parton Densities

7. Limitations of Diffractive Parton Densities

8. Diffractive DIS in Dipole Models

D. Leading Protons and Neutrons Beyond the Pomeron Region

E. Very Hard Diffraction and the BFKL Pomeron

VI. Summary and Outlook

Acknowledgements

References 


\section{INTRODUCTION}

HERA (the Hadron Elektron Ringanlage) (Voss and Wiik, 1994) at the DESY laboratory in Hamburg, Germany is, to date, the only example of a storage ring devoted to producing collisions between leptons and hadrons. It was located in a tunnel of length $6.3 \mathrm{~km}$, housing two independent beam-pipes, the first storing electrons or positrons, here generically referred to as electrons, the second protons. In an initial phase of operation (HERA-I, 1992-2000), the electron beam energy was predominantly $27.5 \mathrm{GeV}$ and the protons were at $820 \mathrm{GeV}(1992-1997)$ or $920 \mathrm{GeV}$ (1998-2000). In its second, higher-luminosity phase (HERA-II, 2002-2007), the electrons remained at the same energy, but were longitudinally polarised, and the proton energy also remained at $920 \mathrm{GeV}$ except for the last few months, when lower energies (575 and $460 \mathrm{GeV}$ ) were used primarily for a measurement of the longitudinal proton structure function. Experiments were located at up to four points around the ring, of which this review is concerned with the two, H1 and ZEUS, where the electrons and the protons were brought to collision, yielding an ep centre-of-mass energy $\sqrt{s} \leq 318 \mathrm{GeV}$.

For the bulk of the kinematic region accessible at HERA, the electron-proton collisions proceed via the exchange of a photon, as shown in Fig. 11. The possible processes are conveniently sub-divided into two categories according to the photon virtuality, $Q^{2}$. The region with $Q^{2} \gg 1 \mathrm{GeV}^{2}$ corresponds to the short distance 'Deep Inelastic Scattering' (DIS) regime, where the photon is usually considered as a structureless $t$-channel exchange. In contrast, in the 'photoproduction' regime, $Q^{2} \rightarrow 0$, the photon can be thought of as a distinct object, decoupling from the electron well in advance of the proton target and interacting as a separate entity, often through its own partonic structure (see Section III.A). To a large extent, analyses at HERA have treated these two kinematic regions separately, though particularly where there are other hard scales in the problem, this is a largely artificial distinction.

Conventionally, the term 'Hadronic Final State' is taken to mean the full final state after the removal of the scattered beam lepton and any electroweak radiation clearly associated with it. It therefore includes any further leptons or gauge bosons produced in the photonproton interaction. As illustrated in Fig. 1, the invariant mass of the hadronic final state is denoted $W$, and is equivalent to the centre-of-mass energy of the $\gamma^{(*)} p$ collision. Expressed in terms of the commonly used 'inelasticity' invariant, $y, W^{2} \approx y s-Q^{2}$. When a hard scale is present, the photon couples to a single quark, which carries a fraction $x$ of the proton's momentum, where $x \approx Q^{2} /(s y)$ is the Bjorken variable. For a more formal introduction to deep inelastic scattering, see for example (Devenish and Cooper-Sarkar, 2004).

The asymmetric beam energies and near-hermetic in-

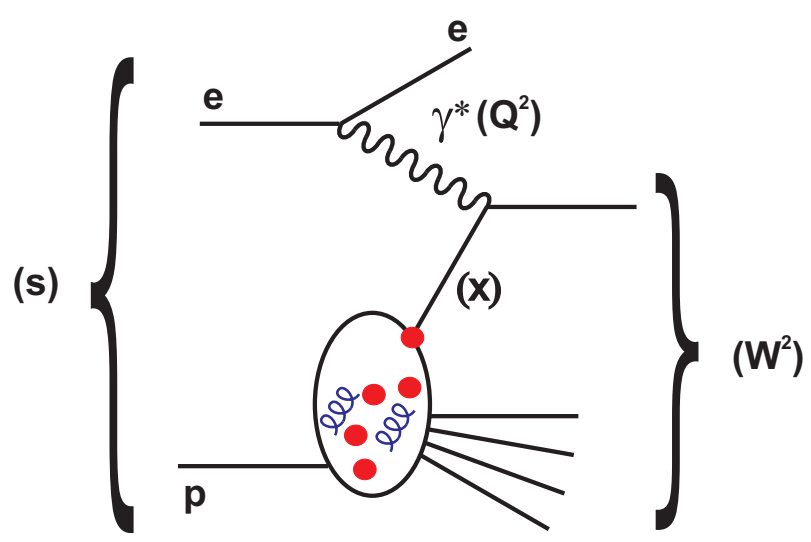

FIG. 1 Schematic illustration of a generic electron-proton DIS process, in which an exchanged photon of virtuality $Q^{2}$ couples to a quark carrying a fraction $x$ of the proton's longitudinal momentum. The electron-proton centre-of-mass energy is denoted $\sqrt{s}$ and the photon-proton centre-of-mass energy (equivalently the invariant mass of the hadronic final state) is denoted $W$.

strumentation of the HERA experiments were well suited to the study of the hadronic final state over a wide range of $W, Q^{2}$ and $x$ values. These favourable kinematics allowed, for the first time, a detailed exploration of the dynamics of the excitations of a single hadron under a multitude of different circumstances. The vast majority of the processes under study are driven by the strong interaction, such that where hard scales are present, the theory of quantum chromodynamics (QCD) (Gross and Wilczek, 1973a, b; Politzer, 1973, 1974) and our understanding of proton and photon structure were tested with unique precision. This has led to new insights and a deeper understanding of QCD in general, as well as providing the stimulus for ever-more sophisticated calculations.

HERA hadronic final state data provide a very wide range in the energy scale of the process. The softest processes are governed by hadronic mass scales of $\mathcal{O}(1) \mathrm{GeV}$. Increasing the scale from this starting point allows the closely-controlled study of the transition from a regime which must be described in terms of hadronic objects to one which can be described perturbatively in terms of partons. At the other extreme, the highest transverse energy jets provide scales of $\mathcal{O}(100) \mathrm{GeV}$, resolving the structure of electron-parton interactions at the $10^{-18} \mathrm{~m}$ level. The corresponding kinematic range in the Bjorken variable is approximately $10^{-4}<x<0.5$. Together with a new window on fragmentation and hadronisation phenomena, these aspects of HERA data have provided a laboratory of unprecedented precision and kinematic range for the elucidation of the QCD dynamics of standard DIS processes, which has led to a vastly improved understanding of the structure of the proton and improved measurements of fundamental parameters of the Standard Model such as the coupling constant of the 
strong force.

Beyond the best understood domain, HERA data have provided access to new kinematic regions and processes with which to test QCD and extend the range of applicability of existing predictions. Many hadronic final state problems have contained multiple hard scales, provided by large transverse momenta and heavy quark masses as well as $Q^{2}$. Understanding the delicate interplay between these different scales has provided exacting challenges to theory. In photoproduction, HERA data scan the transition from the classic ep scattering picture, where the photon interacts in a point-like manner, to the situation where the photon interacts via its own distinct partonic structure, more reminiscent of hadron-hadron scattering, facilitating a controlled study of the differing characteristics of the two extremes. Diffractive and related processes in which the proton stays intact or converts to a neutron via a charge-exchange reaction imply more complex interactions in which no net colour is exchanged. HERA has provided an explosion of precise and eclectic measurements of these hard exclusive and semi-inclusive processes with cleanly identified experimental signatures, leading to a detailed understanding in the framework of QCD. As discussed and illustrated in considerably more detail in the following, the multitude of Hadronic Final State processes studied in $e p$ collisions at HERA has thus led to something of a revolution in the development and testing of QCD.

\section{RECONSTRUCTION OF FINAL STATES AT HERA}

\section{A. The H1 and ZEUS Detectors}

To visualise the physics processes and reconstruction requirements, displays of a neutral and a charged current DIS event are shown in Fig. 2 for the ZEUS (ZEUS, 1993) and H1 (Abt et al., 1997a,b) detectors, respectively. In a neutral current event, where a photon, or at high $Q^{2}$ a $Z^{0}$, is exchanged, a deposit of energy in the calorimeter consistent with an electron, matched to a track, can be seen back-to-back in azimuth with a hadronic jet. In a charged current event, where a $W^{ \pm}$is exchanged, there is no activity to balance the hadronic jet, indicating the presence of an undetected neutrino. The figures also show the key parts of the central detectors. In both cases, the electron beam enters the detector from the left and the proton beam from the right; due to the significantly larger energy of the protons there is more instrumentation in the direction of the outgoing proton beam ${ }^{1}$.

1 A right-handed Cartesian coordinate system is used throughout this review, with the $Z$ axis pointing in the proton beam direction, referred to as the 'forward' direction, and the $X$ axis pointing towards the centre of HERA. The coordinate origin is at the nominal interaction point. The pseudorapidity is defined as $\eta=-\ln \left(\tan \frac{\theta}{2}\right)$, where the polar angle, $\theta$, is measured with

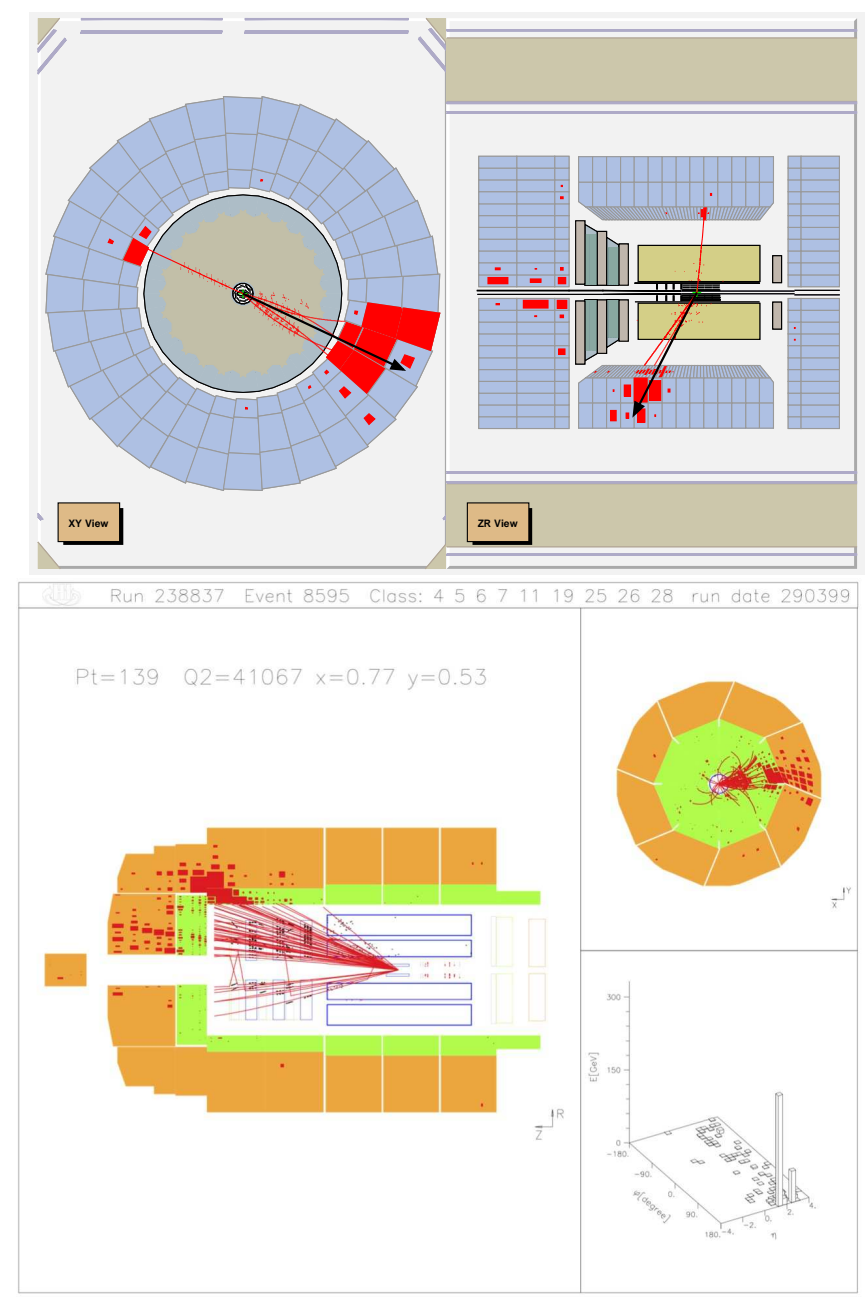

FIG. 2 Event displays of the (top) ZEUS and (bottom) H1 detectors showing a neutral current and a charged current DIS event, respectively.

Other than the forward-backward asymmetry in instrumentation, the detectors were similar to other general-purpose detectors at high energy colliders such as those at LEP, the Tevatron and the LHC. Both H1 and ZEUS detectors had microvertex detectors for measuring weak decays, surrounded by a central drift chamber for the precise tracking of charged particles. Forward and rear tracking detectors gave additional charged-particle information with the rear detectors particularly useful for measuring low-angle scattered electrons at small $Q^{2}$ where the DIS cross section is largest. Beyond the tracking detectors were calorimeters in which a single technology was used for the ZEUS detector with the H1 detector also including a dedicated higher-precision electron calorimeter in the rear direction. Finally, large muon detectors surrounded the calorimeters. Further, smaller

respect to the proton beam direction. 
detectors with dedicated purposes, such as for electron or proton tagging, were placed along the beam-pipe in both directions. These detector components are discussed further in the following subsections.

\section{B. Charged Particles}

Tracks from charged particles were primarily reconstructed by cylindrical drift chambers surrounding the interaction point: central jet chambers (CJC1 and CJC2) (Abt et al., 1997a, b; Burger et al., 1989) in H1 and the central tracking detector (CTD) (Foster et al., 1993, 1994; Harnew et al., 1989) in ZEUS. These were supplemented by silicon vertex detectors (Eick et al., 1997; Pitzl et al., 2000; Polini et al., 2007) placed between the beam-pipe and the drift chambers and in front of forward and rear tracking devices. The H1 and ZEUS drift chambers covered polar-angle regions of $20^{\circ}<\theta<160^{\circ}$ and $15^{\circ}<\theta<164^{\circ}$, respectively, and were operated in uniform axial magnetic fields of $1.16 \mathrm{~T}$ and $1.43 \mathrm{~T}$. The high magnetic fields ensured excellent charge separation up to about $20 \mathrm{GeV}$. The tracking resolutions were both $\sigma\left(p_{T}\right) / p_{T} \approx(0.002-0.003) p_{T}$, with $p_{T}$ in $\mathrm{GeV}$.

Pulse height measurements from the sense wires were also used to calculate the specific energy loss due to ionisation, $\mathrm{d} E / \mathrm{d} x$. The resolutions of the $\mathrm{d} E / \mathrm{d} x$ measurements for well-reconstructed tracks were under $10 \%$ (Bartsch, 2007; Steinhart, 1999). The $\mathrm{d} E / \mathrm{d} x$ measurements were used as a method of particle identification, using likelihood tests to distinguish between electrons, pions, kaons, protons and deuterons.

\section{Electrons, Photons and Muons}

Both detectors had large general purpose sampling calorimeters which covered most of the solid angle for the measurement of electromagnetic (EM) objects. The H1 Collaboration had a finely segmented liquid argon (LAr) (Andrieu et al., 1993) calorimeter with lead or stainless steel absorber, complemented by a lead-scintillating fibre Spaghetti Calorimeter (SpaCal) (Appuhn et al., 1997) which covered polarangle ranges of $4^{\circ}<\theta<153^{\circ}$ and $153^{\circ}<\theta<$ $177^{\circ}$, respectively. Under test beam conditions, the energy resolutions were $\sigma(E) / E \approx 11 \% / \sqrt{E}$ and $\approx 7 \% / \sqrt{E}$ for electrons in the LAr calorimeter and SpaCal, respectively, with $E$ in GeV. The ZEUS detector had a uranium-scintillator calorimeter (Andresen et al., 1991; Bernstein et al., 1993; Caldwell et al., 1992; Derrick et al., 1991) which covered a polar-angle range of $2.5^{\circ}<\theta<178.5^{\circ}$. Under test beam conditions, the single-particle energy resolution for the ZEUS calorimeter was $18 \% / \sqrt{E}$ for electrons, with $E$ in $\mathrm{GeV}$.

One of the primary design considerations of the experiments was the accurate reconstruction of the scat- tered electrons, which should display the signature of isolated high-energy deposits in the EM calorimeter. Various requirements were made on the EM clusters and the isolation, usually including a matching of the clusters of energy to a charged particle track, such that DIS events were selected with high purity and high efficiency (Abramowicz et al., 1995; Glazov, 1998). A typical minimum energy requirement on the scattered electron was $10 \mathrm{GeV}$ for the $\mathrm{H} 1 \mathrm{LAr}$ and ZEUS calorimeters and $6.5 \mathrm{GeV}$ for the SpaCal. In order to make the best measurements of the longitudinal structure function at high $y$, these minimum energy requirements were reduced in dedicated analyses to $3.4 \mathrm{GeV}$ (Aaron et al., 2008c) in the SpaCal and $6 \mathrm{GeV}$ (Chekanov et al., 2009h) in the ZEUS calorimeter.

The variables $Q^{2}, x$ and $y$ can be reconstructed from the energy and polar angle of the scattered electron only, using the so-called "electron method", which arises from simple consideration of the DIS kinematics. Several other reconstruction methods exist which make use of combinations of the measurements of the scattered electron and the hadronic final state (Bassler and Bernardi, 1995; Bentvelsen et al., 1992; Hoeger, 1992; Jacquet and Blondel, 1979). The reconstruction method used depends on the analysis and the regions in which particles are measured; e.g. for charged current events where there is no isolated highenergy electron, the Jacquet-Blondel method was used. Typical relative resolutions in $Q^{2}$ were between about $2 \%$ and $10 \%$, depending on the reconstruction method.

Photon candidates, typically above $5 \mathrm{GeV}$ in transverse energy, were identified in the central calorimeters by reconstructing narrow clusters in the EM calorimeter with no track pointing to them. Backgrounds from $\pi^{0}$ and $\eta$ particles were removed by considering the shape of the cluster and of the calorimeter shower; example quantities were the transverse radius of the cluster and the ratio of the highest energy EM cell to the total cluster energy (Aaron et al., 2010j; Chekanov et al., 2010d). Photons of lower energy could be identified in Compton scattering processes $(e p \rightarrow e \gamma p)$ in which the final state consists only of the photon, an electron and a lack of hadronic activity.

Muons were identified by a combination of tracking, calorimetry and the large muon chambers surrounding the calorimeters. Depending on the given analysis and kinematic cuts and whether a highly pure or highly efficient sample was required, different algorithms were used to combine information from the various sub-detectors. Wherever possible, a track matched with a muon signature in another sub-detector was used for the momentum measurement. A minimum ionising particle (mip) signal in the calorimeters was also used as a high efficiency, but low purity, signal for a muon. Finally, the clearest signal for a muon was provided by the muon chambers. In the case of $\mathrm{H} 1$, the return yoke of the magnetic coil was the outermost part of the detector and was equipped with streamer tubes forming the central muon 
detector (Abt et al. 1 1997b) and tail catcher $\left(4^{\circ}<\theta<\right.$ $\left.171^{\circ}\right)$. In the forward region $\left(3^{\circ}<\theta<17^{\circ}\right)$, a set of drift chamber layers formed the forward muon detector (Biddulph et al., 1994), which allowed a momentum measurement, together with an iron toroidal magnet. The ZEUS central and rear muon chambers (Abbiendi et al., 1993) consisted of limited-streamer tube chambers placed behind the calorimeter, inside and outside a magnetised iron yoke, covering a polar-angle region of $34^{\circ}<\theta<$ $171^{\circ}$. The forward muon detector (Abbiendi et al., 1993) consisted of six trigger planes of limited-streamer tubes and four planes of drift chambers covering the angular region $5^{\circ}<\theta<32^{\circ}$. The efficiency of muon reconstruction depended on the method used, with the detection of a mip signal having almost $100 \%$ efficiency and isolated muons above $2 \mathrm{GeV}$ being detected $90 \%$ and $55 \%$ of the time in the H1 and ZEUS muon systems, respectively (Aaron et al., 2009g).

\section{Heavy Flavour Identification}

As in other high-energy physics experiments, heavy quarks were identified by several means such as reconstructing a given meson mass, requiring a large momentum of a lepton perpendicular to the axis of an associated jet, or reconstructing displaced vertices using a silicon detector placed close the interaction point.

All ground state charm mesons have been reconstructed by combining tracking information from potential decay products. When only considering this information and no further particle identification or measurements of secondary vertices, the decay $D^{*} \rightarrow D^{0} \pi_{s} \rightarrow$ $K \pi \pi_{s}$ has the purest signal; an example is shown in Fig. 3. No beauty mesons have been fully reconstructed due to the significantly smaller statistical sample.

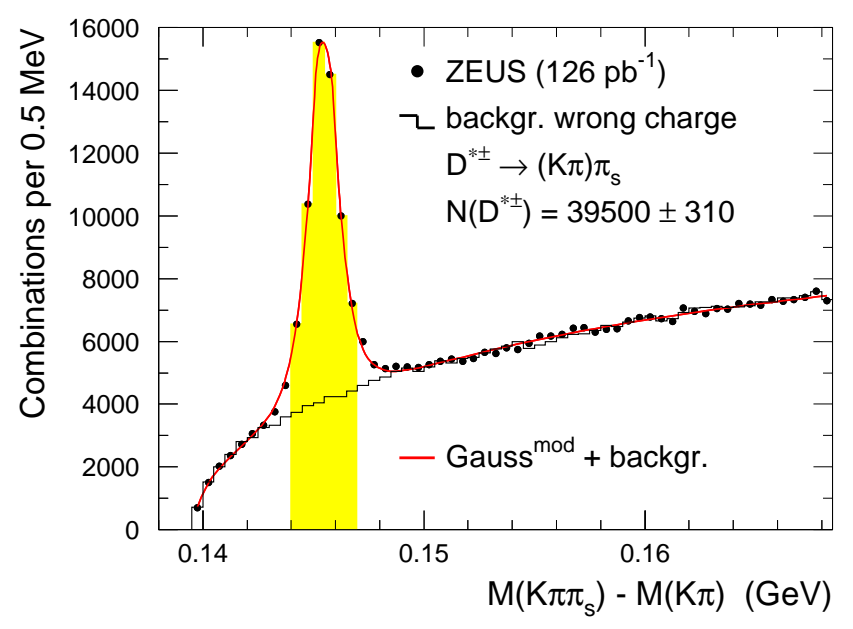

FIG. 3 The distribution of the mass difference $m\left(D^{*}\right)-$ $m\left(D^{0}\right)$ for $D^{*}$ candidates and a background estimate. From (Chekanov et al., 2009i).

Before the use of silicon microvertex detectors, the principal way to identify beauty events was to reconstruct leptons and measure their momentum, $p_{T}^{\text {rel }}$, perpendicular to the axis of an associated jet. This gave a large sample due to a $10 \%$ branching ratio which is much higher than any given meson's branching fraction to easily usable hadronic decays. Due to the quark's larger mass, leptons from beauty decays are concentrated at higher values of $p_{T}^{\text {rel }}$ than leptons from charm decays or in events initiated by light quarks. Typical purities achieved for beauty events by this method were about $20 \%$.

The reconstruction of secondary vertices, using a microvertex detector, from the weak decay of heavy quarks, provides a method for tagging charm and beauty with high efficiency or, by applying stringent requirements, with high purity. The H1 central silicon tracker (CST) (Pitzl et al., 2000) consisted of two layers of double-sided silicon strips, covering an angular range of $30^{\circ}<\theta<$ $150^{\circ}$ for tracks passing through both layers. The ZEUS microvertex detector (MVD) (Polini et al., 2007) consisted of barrel (BMVD) and forward (FMVD) sections with, respectively, three cylindrical layers and four vertical planes. The BMVD provided polar-angle coverage for tracks with three measurements from $30^{\circ}$ to $150^{\circ}$. The FMVD extended the coverage to $7^{\circ}$. A commonly used variable is the impact parameter, $\delta$, of a track which is the transverse distance of closest approach of the track to the primary vertex, see Fig. 4. If the angle $\alpha$ is less than $90^{\circ}, \delta$ is defined as positive, otherwise $\delta$ is defined as negative. Negative values arise only due to imperfect resolution and hence events initiated by light quarks are symmetric about $\delta=0$. An asymmetry to high posi-

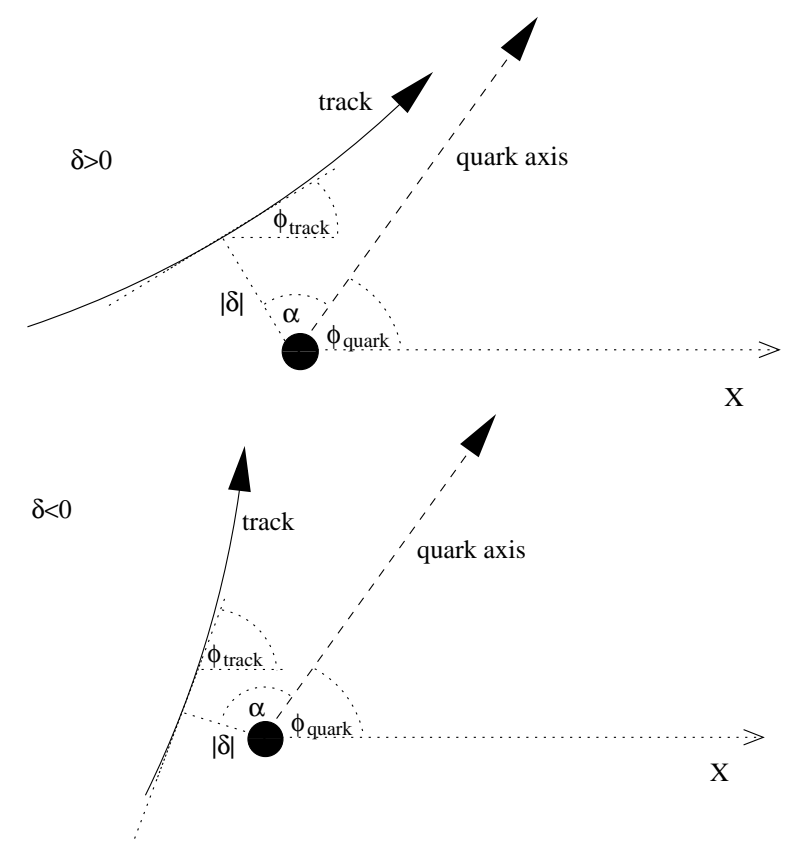

FIG. 4 Diagrams of a track in the $X Y$ plane. The direction of the struck quark, experimentally approximated as a jet axis, is used to define the impact parameter, $\delta$. 
tive values occurs due to heavy quark decays. A more powerful discriminator is the significance, $S_{L}$, at which the transverse distance between the primary and secondary vertices, $L_{x y}$ is non-zero. Thus $S_{L}$ is defined as $L_{x y} / \sigma\left(L_{x y}\right)$, where $\sigma\left(L_{x y}\right)$ is the uncertainty on $L_{x y}$. An

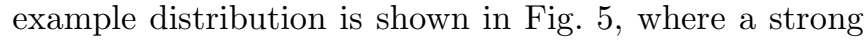
asymmetry is observed.

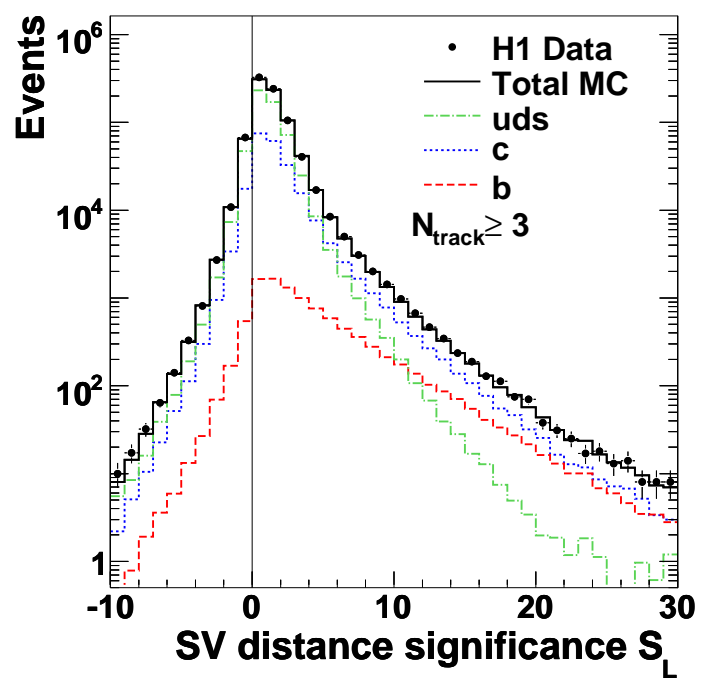

FIG. 5 Distribution of the significance at which the transverse distance between the primary and secondary vertices is nonzero. Included in the figure is a decomposition of the data by fitting Monte Carlo templates for light, $c$ and $b$ quarks. From (Aaron et al., 2010i).

To achieve the most precise measurements for beauty and also charm production, the above and other discriminating variables were combined in a neural network (Aaron et al., 2010i) or a discriminating test function (Chekanov et al., 2008a).

\section{E. Hadronic Jets}

In the first results on jet production from $\mathrm{H} 1$ and ZEUS, jets were reconstructed from the calorimeter cells using the JADE clustering (Bartel et al., 1986; Bethke et al., 1988) or cone algorithms. Due to the theoretical problems with cone algorithms, such as ambiguity in the seed finding and overlapping jets, both collaborations switched to the inclusive $k_{T}$ clustering algorithm (Catani et al., 1993; Ellis and Soper, 1993) for the vast majority of their publications.

Calorimeter cells alone have continued to be used for some publications, due to the finer granularity achieved in the forward parts of the detector compared with the results of using some pre-clustering before the jet finding. However, energy flow objects (EFOs) have also been in common use by both collaborations. These objects are based on an algorithm which combines tracking and calorimeter information in order to optimise the resolution. In a typical jet at HERA, the amount of tracking information used, and hence the improvement in the jet energy resolution when including tracks, is about 10$20 \%$. In certain situations, when several tracks are explicitly reconstructed in a jet, such as a heavy-flavour meson (Chekanov et al., 2009g), the fraction of tracking information used in the EFO input to a jet reaches about $50 \%$ and leads to a significant reduction in the systematic uncertainties such as that due to the calorimeter hadronic energy scale.

The single-hadron energy resolutions, as measured in test beams, are $\sigma(E) / E \approx 50 \% / \sqrt{E} \oplus 2 \%$ and $35 \% / \sqrt{E}$, with $E$ in $\mathrm{GeV}$, for the $\mathrm{H} 1$ and ZEUS calorimeters, respectively. A detailed parametrisation of the jet energy resolution has not been performed. However, a transverse-energy resolution of $9 \%$ has been achieved at H1 for $E_{T}^{\text {jet }}>25 \mathrm{GeV}$ (Caron, 2002) and at ZEUS for $E_{T}^{\text {jet }}>14 \mathrm{GeV}$ (Chekanov et al., 2002a).

The precision of initial measurements of jet cross sections was limited by the uncertainty in the relative jet energy scale between data and Monte Carlo. Due to the cross section rapidly falling with increasing energy, a $1 \%$ uncertainty in the determination of the scale led to about a $5 \%$ uncertainty in the cross section. Therefore, a reduction in the energy scale uncertainty from initial estimates of about $5 \%$ ( $25 \%$ uncertainty in the cross section) was necessary to be able to fully exploit the data for example in precise extractions of parton densities or the strong coupling constant (see Section III.C).

In order to precisely determine the hadronic jet energy scale, various methods were employed, by e.g. using tracking information in a jet or cross-calibrating jets reconstructed in different parts of the calorimeters. However, the use of neutral current DIS events provided the most powerful calibration tool based on the momentum balance between an outgoing jet and the scattered electron. This relied on an accurate reconstruction of the electron and a precise determination of the EM energy scale uncertainty. For the most precise calibrations, closely related techniques exploiting the double angle kinematic reconstruction method (Bentvelsen et al., 1992; Hoeger, 1992) were employed. The difference between the jet and electron transverse energies for data and Monte Carlo simulations are shown for the ZEUS data in Fig. 6] versus the pseudorapidity and transverse energy of the jet. This demonstrates that the uncertainty on the determination of the jet energy scale is $1 \%$ (Chekanov et al., 2002a, d; Wing, 2002). H1 followed similar procedures and achieved an uncertainty of $1.5 \%$ (Aktas et al., 2006h).

\section{F. Photoproduction and DIS and How to Tell Them Apart}

Results from HERA are often (somewhat arbitrarily) classified as pertaining to either photoproduction or DIS. In a DIS event, the exchanged photon is virtual, with 

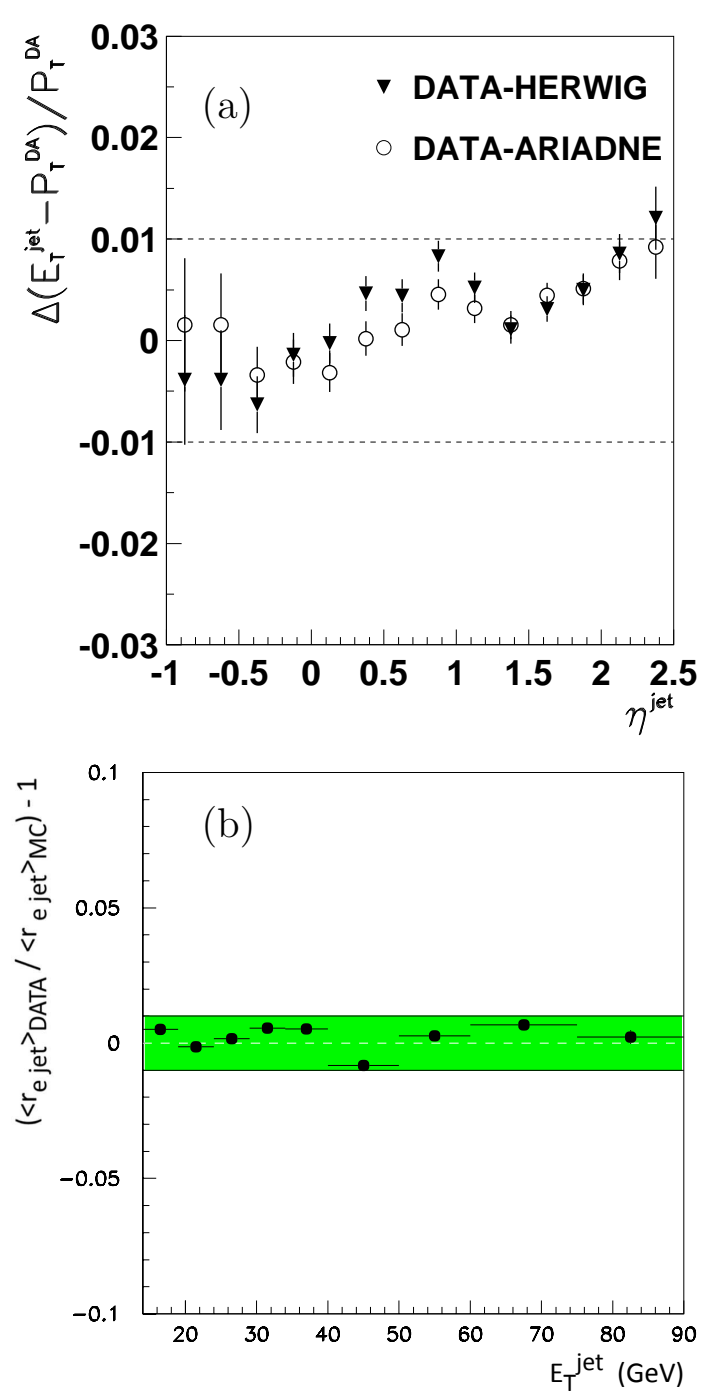

FIG. 6 ZEUS jet energy scale uncertainty as a function of (a) $\eta^{\text {jet }}$ and (b) $E_{T}^{\text {jet }}$, showing that the scale is known to $1 \%$. In (a), the relative difference between the hadronic jet transverse energy, $E_{T}^{\text {jet }}$, and the transverse momentum of the scattered electron, calculated using the double-angle method, $p_{T}^{\mathrm{DA}}$, is shown as a function of the jet pseudorapidity, $\eta^{\text {jet }}$. In (b), the relative difference between the data and Monte Carlo simulation is shown for the quantity $\left\langle r_{e \text { jet }}\right\rangle$ as a function of the jet transverse energy, where $r_{e j e t}$ is the ratio of jet to electron transverse energies.

a squared four-momentum typically larger than about $1 \mathrm{GeV}^{2}$, and a high-energy scattered electron observed in the main calorimeters. Photoproduction is usually defined by the absence of the scattered electron in the main calorimeters, implying a virtuality $Q^{2}<1 \mathrm{GeV}^{2}$ and a median value of $10^{-4}-10^{-3} \mathrm{GeV}^{2}$. The value of $1 \mathrm{GeV}^{2}$ is operational and depends on the exact coverage of the calorimeter. In photoproduction, the photon is quasi-real and the concept of a photon structure is introduced.

Small calorimeters were placed along the direction of the outgoing electron beam at distances up to $40 \mathrm{~m}$ from the interaction point, see Fig. 7 , These were used to tag the electrons in photoproduction events over a narrower range in $Q^{2}$ and $y$. For example, in the case of the $\mathrm{H} 1$ calorimeter at $Z=-33 \mathrm{~m}$, values of $Q^{2}$ were smaller than $0.01 \mathrm{GeV}^{2}$ and the acceptance range was approximately $0.3<y<0.7$. This naturally led to smaller samples compared with those obtained via the anti-tag of an electron in the main calorimeters, but improved the reconstruction resolution.

\section{G. Very Forward Taggers}

Both experiments had very-forward particle taggers to detect low-angle protons and neutrons, particularly for measurements of diffractive production (see Section V).

The H1 forward proton spectrometer (FPS) (Adloff et al., 1999d; Van Esch et al., 2000) and ZEUS leading proton spectrometer (LPS) (Derrick et al., 1997) detected positively charged particles carrying a substantial fraction of the incoming proton energy. The particle trajectories were measured using a system of detectors that could be inserted very close (typically a few $\mathrm{mm}$ ) to the proton beam, positioned between $64 \mathrm{~m}$ and $80 \mathrm{~m}$ and $24 \mathrm{~m}$ and $90 \mathrm{~m}$ from the interaction point for the FPS and LPS, respectively. The leading proton detectors approached the beam in both the horizontal and the vertical planes, with complementary acceptances in scattered proton energy. Despite providing a pure sample of protons, the acceptance was only around $2 \%$. The effective transverse-momentum resolution was dominated by the intrinsic transverse-momentum spread of the proton beam at the interaction point, which was about $40-45 \mathrm{MeV}$ in the horizontal plane and about $100 \mathrm{MeV}$ in the vertical plane.

Both H1 and ZEUS had forward neutron calorimeters (Adloff et al., 1999d; Bhadra et al., 1995, 1997a,b) installed in the HERA tunnel at $\theta=0$ and at $Z=106 \mathrm{~m}$ from the interaction point in the protonbeam direction. Both devices consisted of a main calorimeter, with hadronic energy resolution of $\sigma(E) / E \approx(60-70) \% / \sqrt{E}$, with $E$ in $\mathrm{GeV}$, supplemented by a pre-shower calorimeter in the case of $\mathrm{H} 1$ and a scintillator hodoscope in the case of ZEUS. The forward neutron calorimeters had a limited angular coverage, being sensitive to neutrons of less than about $0.05^{\circ}$ or $0.8 \mathrm{mrad}$. Both devices achieved spatial resolutions of about $2 \mathrm{~mm}$.

Figure 7 shows an illustration, for the example of the $\mathrm{H} 1$ experiment, of the layout of the additional components along the beamline in both directions away from the main detector.

\section{H. Triggering}

Both H1 (Baird et al., 2001; Nicholls et al., 1998; Sefkow et al., 1995) and ZEUS (Allfrev et al., 2007; 


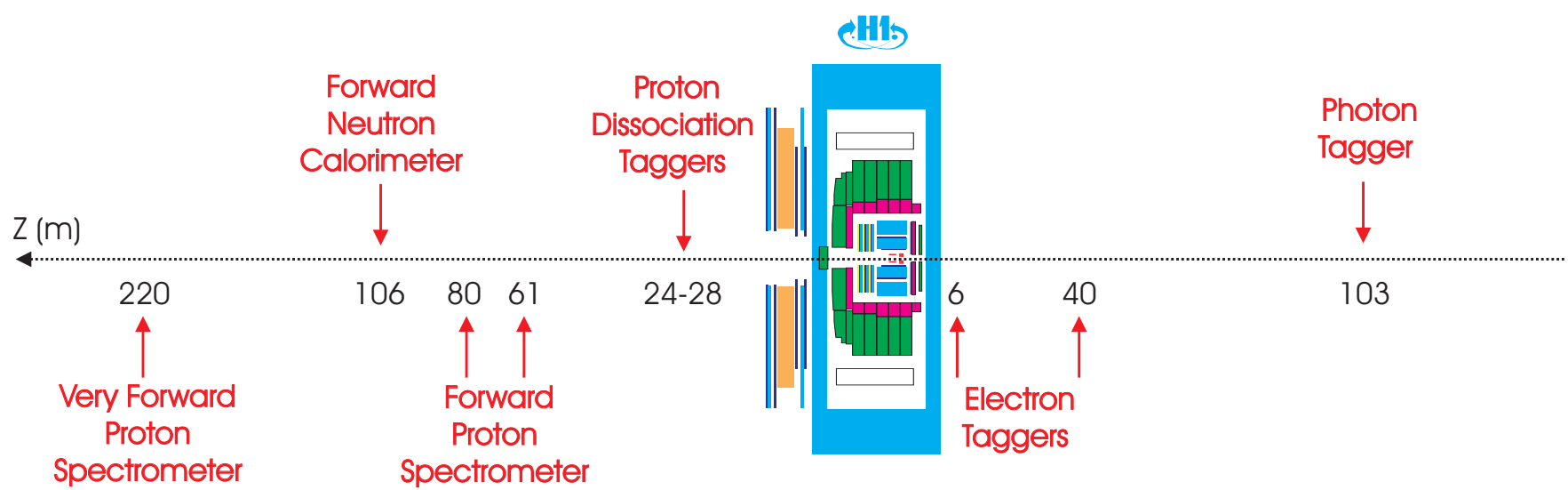

FIG. 7 Schematic illustration of the beamline instrumentation for the example of the H1 experiment. The detector components shown are all described in the text with the exception of the $103 \mathrm{~m}$ photon tagger which was used for luminosity determination via the Bethe-Heitler $e p \rightarrow e p \gamma$ process and the proton dissociation taggers which were used to identify forward rapidity gaps (see Section V).

Smith et al., 1992) experiments mainly used a three-level trigger system of progressive sophistication in order to select the most interesting events on-line and remove background from beam-gas interactions and low energy ep collisions in which additional statistics would not improve any measurement. The H1 trigger consisted of two hardware levels and a software filter farm, whereas ZEUS had one hardware level and two software filters. The first two levels often considered simple energy sums in the calorimeter or the reconstructed vertex position. At the third level, a simplified and fast version of much of the offline reconstruction code, such as jet and tracking algorithms, was used. From a nominal HERA bunch crossing rate of $10 \mathrm{MHz}$, the trigger system reduced the rate such that data were written to storage at a rate of $\approx 10 \mathrm{~Hz}$.

Trigger filters which selected events with a high-energy electron candidate were very efficient selectors of DIS events. Due to bandwidth restrictions, these were often pre-scaled or were combined with additional requirements, such as a jet or a reconstructed meson in the event, depending on the physics motivation. Several hundred different combinations of triggers existed to try and cover all interesting physics channels, whilst remaining within the limits dictated by data transfer rates and processing speeds.

The principal trigger chains in the $\mathrm{H} 1$ and ZEUS experiments, both in terms of bandwidth and frequency of use in physics analyses, were designed to select inclusive DIS and jet events. The information to select an electron or jet candidate was often supplemented in the trigger with tracking information and, in the case of the photoproduction selection at $\mathrm{H} 1$, with an electron tag in the beam-pipe calorimeters.

\section{HADRONIC FINAL STATES AND SHORT DISTANCE PHYSICS}

In this section, measurements are presented of the hadronic final state in the presence of a hard scale, typically the virtuality, $Q^{2}$, of the exchanged boson, the transverse energy of a jet or the mass of a heavy quark in the event. A high or hard scale implies that the reaction occurs at short distance and hence allows perturbative calculations to be performed. Almost all processes and cross sections at HERA, both DIS and photoproduction, involving a jet of high transverse energy or a heavy quark have been calculated in the DGLAP Altarelli and Parisi, 1977; Dokshitzer, 1977; Gribov and Lipatov, 1972; Lipatov, 1975) approximation to next-to-leading order (NLO) in QCD perturbation theory. In many cases, several different approaches to the NLO QCD calculations are available for a given process and input parameters such as the strong coupling constant can also be varied. Comparisons between the calculations and with data have led to improvements in the predictions. Various other approximations to QCD are also available which emphasise different aspects of parton cascade dynamics and evolution. These include the BFKL (Balitsky and Lipatov, 1978; Kuraev et al., 1976, 1977) and CCFM (Catani et al., 1990a,b; Ciafaloni, 1988; Marchesini, 1995) approaches. A thorough review of nonDGLAP predictions of standard short distance processes is not carried out here since NLO corrections are not usually available. However, these approaches are considered later in the context of low $x$ physics (Section III.D) and diffraction (Section V).

Measurements of jet and heavy flavour production are directly sensitive to the gluon density in the proton and have hence been used in QCD fits to constrain the parton densities in the proton. Heavy quark data in DIS are also used to extract the beauty, $F_{2}^{b \bar{b}}$, and charm, $F_{2}^{c \bar{c}}$, contri- 
bution to the proton structure, $F_{2}$. These results are reviewed in detail elsewhere (Klein and Yoshida, 2008; Perez and Rizvi, 2013). Measurements of photoproduction are also sensitive to the structure of the photon and the data can in principle be used in fits to constrain the parton densities in the photon. A more detailed review of photoproduction and its constraints on photon structure can also be found elsewhere (Butterworth and Wing, 2005); however, new results since that review are discussed here.

\section{A. Perturbative QCD Theory of the Hadronic Final State}

A brief description of perturbative QCD related to the hadronic final state is given in this section. Fuller accounts can be found elsewhere (Brock et al., 1995; Dissertori et al., 2003; Ellis et al., 1996).

Given that the lowest-order DIS process, a quarkparton model (QPM) event (see Fig. 11), contains a scattered electron recoiling against a jet, it may seem trivial to describe jet cross sections in DIS. However, once the sizeable phase space for parton radiation is considered in the context of the wide range of possible jet algorithms, the situation becomes far more subtle. Jet cross sections are generally presented in the Breit frame Fevnman, 1972; Streng et al., 1979) in which the exchanged virtual boson is purely space-like, with 3-momentum $\boldsymbol{q}=$ $(0,0, Q)$, and is collinear with the incoming parton, such that QPM events do not contribute at large transverse energies. Therefore leading-order (LO) QCD processes, Fig. 8 dominate jet cross sections in DIS.

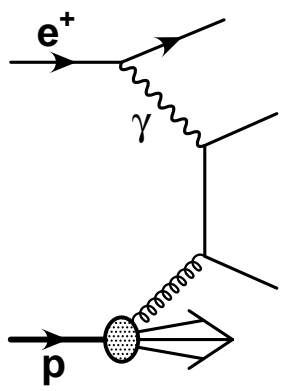

(a)

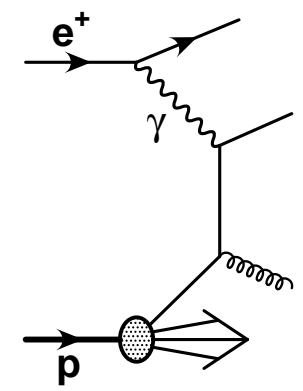

(b)
FIG. 8 Illustrations of the (a) boson-gluon-fusion and (b) $u$-channel QCD Compton processes. Along with $s$-channel QCD Compton scattering, these are the LO QCD processes in DIS and direct photoproduction, i.e. the lowest-order process involving at least one power (or vertex) of $\alpha_{s}$.

From the diagrams, it can be seen that the bosongluon-fusion process is related to the gluon density in the proton. This is dominant at low $Q^{2}$, where low- $x$ partons are most important, whereas the QCD Compton process becomes more important with increasing $Q^{2}$ since it is related to the quark density in the proton. Measurements of jet cross sections are therefore sensitive to the strong coupling constant, $\alpha_{s}$. When combined with inclusive DIS cross-section measurements, they allow its precise extraction simultaneously with the parton densities in the proton, as discussed in Section [II.C This can be seen from a general schematic formula for perturbative QCD calculations of DIS jet processes:

$$
\begin{gathered}
\mathrm{d} \sigma_{e p \rightarrow e+\mathrm{jets}+X}=\sum_{a} \int_{0}^{1} \mathrm{~d} \hat{\sigma}_{e a \rightarrow c d}\left(x, \alpha_{s}\left(\mu_{R}\right), \mu_{F}, \mu_{R}\right) \\
f_{a / P}\left(x, \mu_{F}\right) \mathrm{d} x,
\end{gathered}
$$

where the sum is over the possible partons, $a$, in the proton given by the parton density function (PDF), $f_{a / P}$. The factorisation and renormalisation scales are denoted by $\mu_{F}$ and $\mu_{R}$ and may be given by $\sqrt{ } Q^{2}$, the jet transverse energy, or a combination of the two. The shortdistance cross section, $\mathrm{d} \hat{\sigma}_{e a \rightarrow c d}$, depends on $x$, the strong coupling, $\alpha_{s}, \mu_{F}$ and $\mu_{R}$.

In photoproduction, where the electron escapes detection and continues down the beam-pipe, the virtuality, $Q^{2}$, is low and the hard scale is given instead by the transverse energy of the jets. The diagrams shown in Fig. 8 also apply to the LO direct jet photoproduction process where direct-photon events are classified as those in which all of the photon's momentum participates in the hard interaction. Equation 1 is modified to the general formula :

$$
\begin{gathered}
\mathrm{d} \sigma_{e p \rightarrow e+\mathrm{jets}+X}=\sum_{a} \int_{0}^{1} \mathrm{~d} \hat{\sigma}_{\gamma a \rightarrow c d}\left(x, \alpha_{s}\left(\mu_{R}\right), \mu_{F}, \mu_{R}\right) \\
f_{\gamma / e} f_{a / P}\left(x, \mu_{F}\right) \mathrm{d} x,
\end{gathered}
$$

where the term $f_{\gamma / e}$ represents the probability of the electron radiating a photon and is given by the WeizsäckerWilliams formula (Frixione et al., 1993; von Weizsacker, 1934; Williams, 1934). Another class of events, resolvedphoton processes, also contribute to the photoproduction cross section. At LO, such processes are classified as those in which only a fraction of the photon's momentum participates in the hard interaction. For such events, the photon can be considered as developing a structure, the parton densities of which are probed by the hard scale of the interaction. This means that the $e p$ collision can be viewed as a hadron-hadron collision in which partons from both the photon and the proton participate in the hard process. Therefore many extra diagrams contribute in LO QCD to the photoproduction cross section; an example is shown in Fig. 9, in which a quark from the photon collides with a gluon from the proton.

A general schematic formula for perturbative QCD calculations of photoproduction processes is given by: 


$$
\mathrm{d} \sigma_{e p \rightarrow e+\text { jets }+X}=\sum_{a, b} \int_{0}^{1} \mathrm{~d} x_{\gamma} \int_{0}^{1} \mathrm{~d} x_{p} f_{\gamma / e} f_{b / \gamma}\left(x_{\gamma}, \mu_{F \gamma}\right) f_{a / p}\left(x_{p}, \mu_{F p}\right) \mathrm{d} \hat{\sigma}_{a b \rightarrow c d}\left(x_{\gamma}, x_{p}, \alpha_{s}\left(\mu_{R}\right), \mu_{F \gamma}, \mu_{F p}, \mu_{R}\right),
$$

where $x_{p}$ and $x_{\gamma}$ are the longitudinal momentum fractions of the parton $a$ in the proton and the parton $b$ in the photon, respectively. The term $f_{a / p}\left(f_{b / \gamma}\right)$ represents the PDFs of partons with flavour $a(b)$ in the proton (photon). The factorisation scale for the proton (photon) is denoted by $\mu_{F p}\left(\mu_{F \gamma}\right)$, and $\mu_{R}$ is the renormalisation scale. The factorisation and renormalisation scales are often assumed to have the same value in calculations, although this is not necessarily the case and hence for generality, they are here treated separately. The term $d \hat{\sigma}_{a b \rightarrow c d}$ is the hard (partonic) cross section. In the case where parton $b$ is the entire photon, $f_{b / \gamma}\left(x_{\gamma}, \mu_{F \gamma}\right)$ is $\delta\left(1-x_{\gamma}\right)$ and Eq. 3 describes direct photoproduction and reduces to Eq. [2]

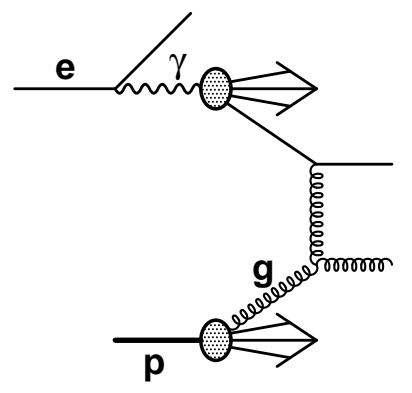

FIG. 9 An example of a LO resolved jet photoproduction process, containing a hard scattering between a quark from the photon and a gluon from the proton.

The separation between resolved and direct processes has more to do with the limitations of our ability to calculate QCD cross sections than with fundamental physics. The separations are not unique beyond LO. For example the LO resolved-photon process in Fig. 9 can also be considered as a direct-photon process in NLO QCD. Nevertheless, the labels 'direct' and 'resolved' are useful tools for exploring the world of photon physics.

The NLO corrections for the production of two hard partons at HERA, both in photoproduction and DIS, were calculated in the 1990s (see below), thereby allowing comparisons with inclusive-jet and dijet measurements, by applying a jet algorithm to the partons in the final state of an NLO parton-level event generator. These NLO QCD calculations generally give an accurate prediction of the normalisation and the shapes of basic kinematic distributions. However, in order to compare with observables measurable from the data, corrections for hadronisation using Monte Carlo models are necessary. A multiplicative hadronisation correction factor is determined from the ratio of the cross sections at the hadron and parton levels in the Monte Carlo simulation. As the simulations are only based on LO matrix elements with parton showering, their applicability is questionable. However, some level of control over the procedure can be assured by checking the compatibility of the dependences on important variables such as jet transverse energy and angle between the NLO calculation and the parton level Monte Carlo simulation. For some event properties and kinematic configurations, the NLO QCD calculations are not very reliable, due to the fact that they only allow at most one parton to be radiated in addition to the primary jet pair. Calculations at the next order, next-to-next-to-leading order (NNLO) in QCD (Alekhin et al., 2010; Jimenez-Delgado and Reya, 2009; (Martin et al., 2009), have been performed for inclusive DIS but not with final-state objects such as jets or heavy quarks present.

In DIS, NLO QCD calculations are available for the production of jets in neutral current (Catani and Sevmour, 1997; Graudenz, 1997; Mirkes and Zeppenfeld, 1996; Nagy and Trocsanvi, 2001; Potter, 1999) and charged current (Mirkes and Zeppenfeld, 1996) processes. The NLO corrections have also been calculated for $2 \rightarrow 3$ scattering (i.e. three-jet cross sections) in DIS (Nagy and Trocsanyi, 2001) and can in principle be extended to photoproduction. Inclusive hadron production has also been calculated to NLO (Albino et al., 2005, 2008; de Florian et al., 2007a,b; Kniehl et al., 2000; Kretzer, 2000), whereas prompt photon production has been calculated to $\mathcal{O}\left(\alpha^{3}\right)$ (Gehrmann-De Ridder et al., 2006a, b, 2000).

In photoproduction, NLO QCD calculations are available for the production of jets (Aurenche et al. 2000; Frixione, 1997; Frixione et al., 1996; Frixione and Ridolfi, 1997; Gordon and Storrow, 1992; Harris and Owens, 1997; Klasen and Kramer, 1997), hadrons (Binnewies et al., 1995; Fontannaz et al., 2002), and prompt photons (Fontannaz et al., 2001; Fontannaz and Heinrich, 2004; Gordon and Storrow, 1994; $\quad$ Krawczvk and Zembrzuski, 2001; Zembrzuski and Krawczyk, 2003).

The above perturbative calculations all require some choices of input parameters, and also need to be corrected for hadronisation, which lead to uncertainties in the predictions. The renormalisation and factorisation scales, the proton and photon PDFs, the value of $\alpha_{s}$ and, where appropriate, fragmentation functions all need to be chosen. The uncertainties are usually dominated by varying the renormalisation scale by a factor of two. However they vary depending on the phase space and distribution 
measured; the precision of the predictions are discussed where appropriate in the following sections. It should be noted that the scale variation by a factor of two is merely convention and bears no relation to e.g. a onesigma uncertainty. This should therefore be treated with caution. In one example fit to data (Chekanov et al., 2005a), the variation produced unacceptable $\chi^{2}$ values and so a variation of $\sqrt{2}$ was chosen. Measurements in which variation of the scale by a factor of two appear to be an under-estimation are discussed in Section III.D.

The above parton-level calculations for jet production assume massless partons, which is also a possible procedure when calculating heavy-quark production (the socalled "massless" scheme (Binnewies et al., 1997, 1998; Kniehl et al., 1997)). These calculations are for photoproduction; no heavy flavour calculations exist using this scheme for DIS. Here charm and beauty are regarded as active flavours in the PDFs of the proton and photon and are fragmented from massless partons into massive hadrons after the hard process. This scheme should be applicable at high transverse momenta. For momenta of the outgoing heavy quark of the order of the quark mass, the fixed-order or "massive" scheme in photoproduction (Frixione et al., 1995a,b) and in DIS (Harris and Smith, 1995a, b, 1998) is more appropriate. In the massive scheme, $u, d$ and $s$ are the only active flavours in the structure functions of the proton and charm and beauty are produced only in the hard subprocess. Compared with inclusive jet production, these calculations are subject to significant additional uncertainties from the mass of the heavy quark and, where appropriate, the transition of the quark to a hadron. More details of the different schemes and in particular their relevance for determination of PDFs is given in (Abramowicz et al., 2013a) and references therein.

In addition to NLO calculations, data are also often compared with predictions from Monte Carlo models which incorporate LO matrix elements matched with leading-logarithm parton showers. The Monte Carlo models generally give a more complete and realistic final state, but are unreliable in normalisation due to the fact that the matrix elements are currently only LO. The approaches (Frixione and Webber, 2002; Nason, 2004) of matching matrix elements calculated at NLO with parton showers is widely used for LHC processes. This has been extended to HERA physics but only for heavy flavour production (Toll, 2010).

\section{B. Comparisons with Data}

\section{Jet Production}

A measurement of the inclusive jet cross section is shown as a function of the jet transverse energy in the Breit frame, $E_{T, \mathrm{~B}}^{\mathrm{jet}}$, in Fig. 10. The cross section falls by three orders of magnitude as $E_{T, \mathrm{~B}}^{\mathrm{jet}}$ increases from $9 \mathrm{GeV}$ to $50 \mathrm{GeV}$ and the uncertainty on the measurement re- mains below $5 \%$ for $E_{T, \mathrm{~B}}^{\mathrm{jet}}<30 \mathrm{GeV}$, dominated by the uncertainty on the jet energy scale. The statistical uncertainties only become dominant above $30 \mathrm{GeV}$. The excellent description of these data is a triumph of QCD. The theoretical uncertainties are of broadly similar size to those from experiment, though larger at low $E_{T, \mathrm{~B}}^{\text {jet }}$ and smaller at larger $E_{T, \mathrm{~B}}^{\mathrm{jet}}$.

Figure 10(a) illustrates the need to specify the choice of algorithm when discussing jet cross sections. The quality of the theoretical description is approximately the same for the $k_{T}$ (Catani et al., 1993), anti- $k_{T}$ (Cacciari et al., 2008) and SIScone (Salam and Soyez, 2007) algorithms, though the cross sections themselves and the necessary hadronisation corrections are algorithm-dependent. Figure 10(a) shows that theory describes the data for all jet algorithms in this large $Q^{2}$ range. In Fig. 10(b), H1 data for measurements using the $k_{T}$ algorithm are shown at lower and in different regions of $Q^{2}$; the NLO QCD prediction also describes the data well here. In general, measurements of inclusive-jet (Aaron et al., 2010e. h; Abramowicz et al., 2010b; Adloff et al., 2001b, 2002e; Aktas et al., 2007d; Chekanov et al., 2002e, 2003f, 2007g.h), dijet (Aaron et al., 2010g,h; Abramowicz et al., 2010a; Adloff et al., 2001b; Breitweg et al., 2001a; Chekanov et al., 2002b, 2007g) and trijet (Aaron et al., 2010g.h; Adloff et al., 2001d; Chekanov et al., 2005e, 2012) production in DIS are all well described by NLO QCD, particularly at high $E_{T}$ or high $Q^{2}$ and at central values of pseudorapidity. Such a description of the kinematic trends of jet production in DIS allows an extraction of the parton densities in the proton and/or the value of the strong coupling constant to be made, as discussed in Section III.C.

Jet photoproduction has the added possibility for the photon to develop a structure and so the data can in principle be used to extract information on this. The structure, or more precisely the parton densities, of the photon are generally extracted from measurements of DIS $e \gamma$ interactions at $e^{+} e^{-}$colliders (Nisius, 2000). However, due to the $Q^{-4}$ dependence of the $e p$ cross section, measurements of photoproduction at HERA offer larger statistics than are available from $e^{+} e^{-}$data, as well as probing higher energy scales. Jet photoproduction is reviewed in more detail elsewhere (Butterworth and Wing, 2005), with a few of the highlights and new results included here.

Some of the first measurements at HERA established the potentially hard scattering nature of photoproduction through the observation of two jets with significant transverse energy (Ahmed et al., 1992; Derrick et al., 1992) in events in which no scattered electron was observed in the main detectors. The need for both directand resolved-photon interactions in describing photoproduction at HERA was also shown by comparing data with models of just one of the processes or the combined prediction. A variable particularly sensitive to the nature of the photon and the relative fraction of directand resolved-photon processes is a hadron- or detector- 

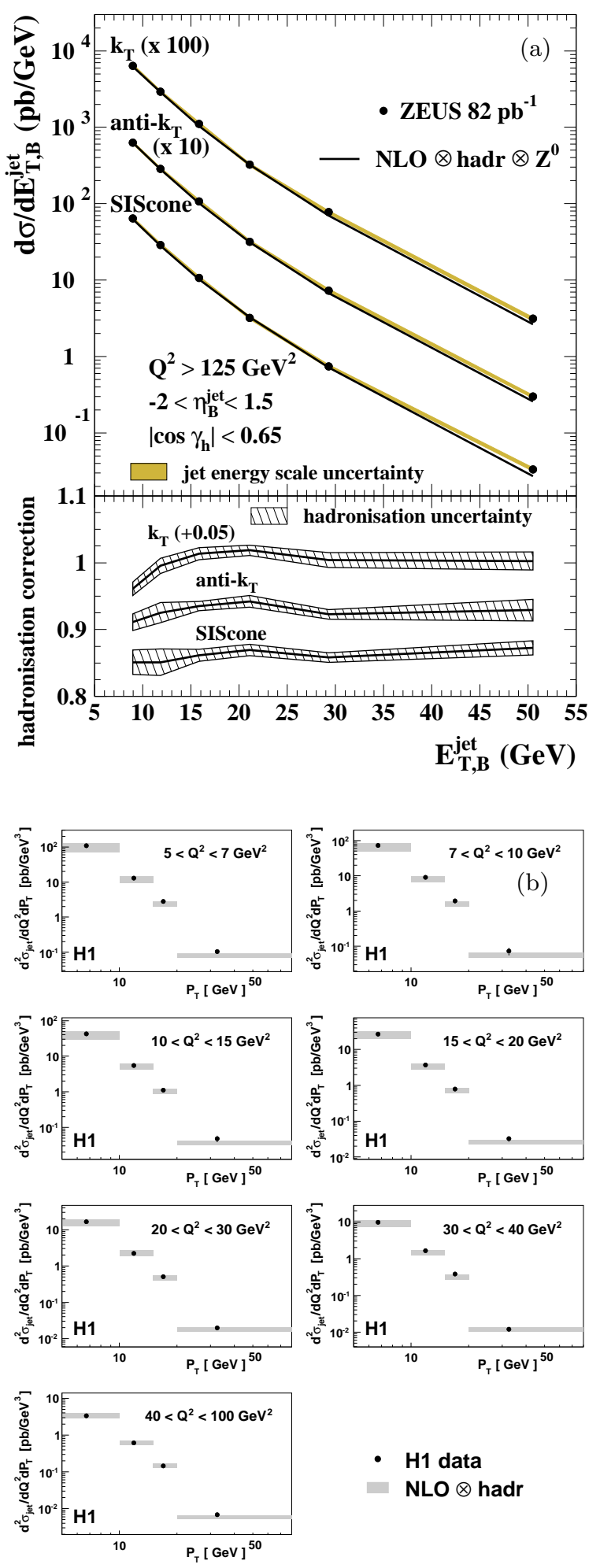

- H1 data
NLO $\otimes$ hadr

FIG. 10 (a) Measurement of $\mathrm{d} \sigma / \mathrm{d} E_{T, B}^{\text {jet }}$ for inclusive-jet production in the Breit Frame in DIS for different jet algorithms. The lower part of the figure shows the hadronisation corrections applied to the NLO calculations. (b) Measurement of $\mathrm{d}^{2} \sigma / \mathrm{d} Q^{2} \mathrm{~d} P_{T}$ in different regions of $Q^{2}$ for inclusive-jet production in the Breit Frame in DIS using the $k_{T}$ jet algorithm. Note that $P_{T}$ is equivalent to $E_{T, B}^{\mathrm{jet}}$. The data in (a) and (b) are compared with NLO QCD predictions (corrected for hadronisation and $Z^{0}$ effects). (a) From (Abramowicz et al., 2010b) and (b) from (Aaron et al., 2010e). level estimator of $x_{\gamma}$, the photon's momentum fraction which takes part in the hard scatter. At LO, $x_{\gamma}$ is identically equal to 1 for direct-photon processes and less than 1 for resolved-photon processes. Experimentally (Derrick et al., 1994a) the $x_{\gamma}$ estimator was reconstructed as

$$
x_{\gamma}^{\text {meas }}=\frac{\sum_{\text {jets }}\left(E-p_{z}\right)_{\text {jets }}}{\sum_{i}\left(E-p_{z}\right)_{i}}
$$

where the sums in the numerator and denominator run over all jets and all energy deposits in the calorimeter, respectively. The first measurement of a distribution in this quantity is shown in Fig. 11 and is compared with a two component fit using direct and resolved photon templates from the HeRwig (Corcella et al., 2001; Marchesini et al., 1992) Monte Carlo programme. The data exhibit a two-peak structure at high and low values of $x_{\gamma}^{\text {meas }}$. The direct- and resolved-photon components in the Monte Carlo have very different shapes. The Monte Carlo prediction gives a reasonable representation of the data when direct- and resolved-photon processes are added together. The resolved-photon component describes the low $x_{\gamma}^{\text {meas }}$ region reasonably well but cannot describe the data at high $x_{\gamma}^{\text {meas }}$. These data at high $x_{\gamma}^{\text {meas }}$ can only be described with the inclusion of the component from direct-photon processes. Hence these data constituted the first observation of direct-photon processes in photoproduction.

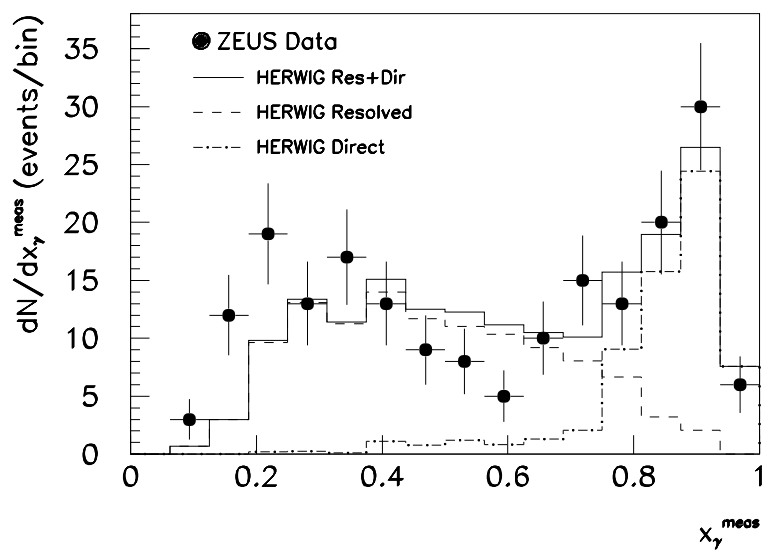

FIG. 11 Raw distribution in $x_{\gamma}^{\text {meas }}$ for photoproduction events with two or more jets. The direct- and resolved-photon Monte Carlo predictions are fitted to the data with free normalisation. From (Derrick et al., 1994a).

After these initial findings, a multitude of results from both collaborations were published, investigating the ability of NLO QCD to describe jet photoproduction data (the reader is referred to the H1 (H1, 1992-) and ZEUS (ZEUS, 1992-) paper lists). These studies addressed the need for an underlying event due to secondary scatters and yielded extractions of $\alpha_{s}$ and measurements of quantities sensitive to the structure 
of the proton and photon. The most recently published (Abramowicz et al., 2012a; Aktas et al., 2006h; Chekanov et al., 2007f) and most precise measurements of jet photoproduction focus on high transverse energy so as to minimise the effects of any underlying event and therefore to provide a clean probe of the structure of the proton and photon.

Figure 12 shows the distribution of the mean transverse energy of the two highest- $E_{T}$ jets, $\bar{E}_{T}$, in two regions of $x_{\gamma}^{\text {obs }}$ (Derrick et al., 1995a), defined as

$$
x_{\gamma}^{\mathrm{obs}}=\frac{\sum_{\mathrm{jet}} E_{T}^{\mathrm{jet}} e^{-\eta^{\mathrm{jet}}}}{2 y E_{e}},
$$

which is closely related to $x_{\gamma}^{\text {meas }}$, with the high- $x_{\gamma}^{\text {obs }}$ region enriched in direct-photon events and low $x_{\gamma}^{\text {obs }}$ enriched in resolved-photon events. At the high transverse energies measured here the high- $x_{\gamma}^{\text {obs }}$ region is well described by NLO QCD, although a difference in shape is observed between data and theory. As can be seen from the predictions using two rather different photon PDFs, AFG04 (Aurenche et al., 2005) and CJK (Cornet et al., 2004), the sensitivity to the structure of the photon is

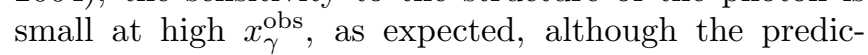
tion using CJK describes the data somewhat better. For $\bar{E}_{T}<40 \mathrm{GeV}$, the dominant uncertainty on the data is due to the jet energy scale (see Section II.E), which will not be improved in future measurements. In this region, the uncertainties on the data are also significantly smaller
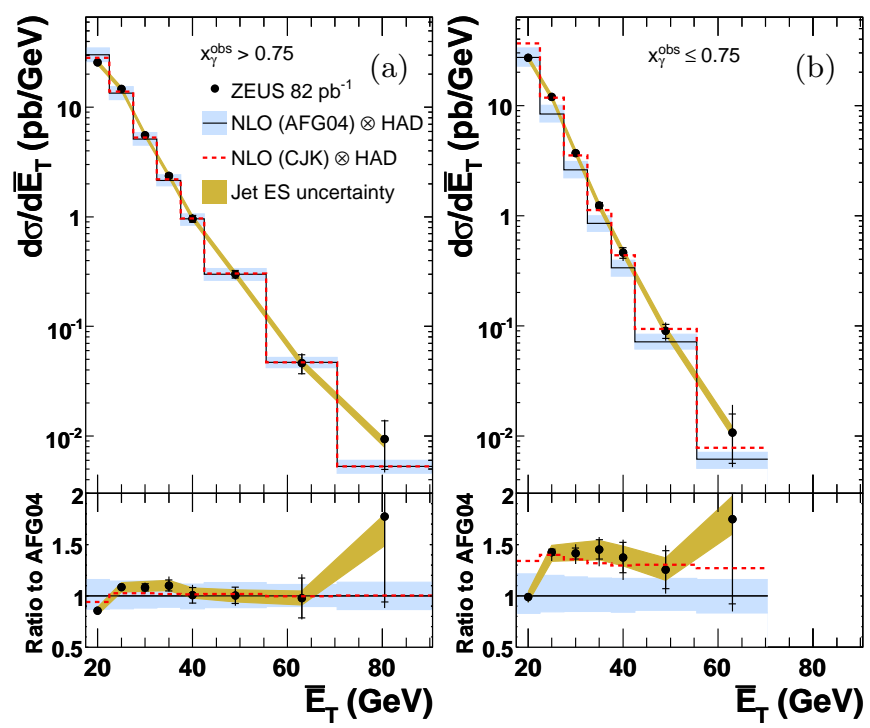

FIG. 12 Measured cross-section $\mathrm{d} \sigma / \mathrm{d} \bar{E}_{T}$ for (a) $x_{\gamma}^{\text {obs }}>0.75$ and (b) $x_{\gamma}^{\text {obs }}<0.75$, compared with NLO QCD predictions (corrected for hadronisation) using photon PDFs, AFG04 (solid line) and CJK (dashed line). From (Chekanov et al., 2007f).

At low $x_{\gamma}^{\text {obs }}$, the difference in shape between data and NLO QCD in Fig. 12(b) is more marked. For the cal- culations using AFG04, the data and NLO QCD prediction agree in the lowest bin whereas the prediction is significantly below the data at high $\bar{E}_{T}$. In contrast, the prediction from CJK is too high in the first bin, which dominates the cross section, but agrees well at higher $\bar{E}_{T}$. Although the prediction from CJK clearly lies above the data, it also gives the best description of the dependence on other variables such as the average pseudorapidity of the jets, $\bar{\eta}$ (Chekanov et al., 2007f). All other parametrisations of the photon PDFs give a qualitatively similar description of the data to that of AFG04. The fact that the gluon density in the CJK photon PDF differs from the others may hint at the origin of the improved description. These data should thus improve our knowledge of the gluon density in the photon PDFs, which is insufficiently constrained by $e^{+} e^{-}$data.

\section{Jet Substructure}

The substructure of a jet gives information on the internal pattern of parton radiation, as well as details of the hadronisation process. Given that gluons radiate more than quarks, categorising jets using measurements of their substructure offers the possibility of obtaining samples which are enriched in gluon or quark initiators. Classifying the jets in an event using this technique thus allows samples to be obtained which are dominated by particular parton-level final states, in turn giving enhanced control over the initial state. Measurements of jet substructure could thus in principle lead to improved constraints on the structure of the proton and photon, distinguishing for example between the $\gamma^{*} g \rightarrow q \bar{q}$ and $\gamma^{*} q \rightarrow q g$ processes in the DIS case.

Jet substructure is generally studied by measuring the jet shape (Ellis et al., 1992) and subjet multiplicity (Catani et al., 1992; Forshaw and Sevmour, 1999; Sevmour, 1994, 1996). The integrated jet shape, $\psi(r)$, using only those particles belonging to the jet, is defined as the fraction of the jet transverse energy that lies inside a cone in the $\eta-\phi$ plane of radius, $r$, concentric with the jet axis :

$$
\psi(r)=\frac{E_{T}(r)}{E_{T}^{\mathrm{jet}}},
$$

where $E_{T}(r)$ is the transverse energy within the given cone of radius $r$. The mean integrated jet shape, $\langle\psi(r)\rangle$, is defined as the averaged fraction of the jet transverse energy inside the cone $r$ :

$$
\langle\psi(r)\rangle=\frac{1}{N_{\text {jets }}} \sum_{\text {jets }} \frac{E_{T}(r)}{E_{T}^{\text {jet }}},
$$

where $N_{\text {jets }}$ is the total number of jets in the sample. The substructure of a jet is expected to depend primarily on the initiator of the jet and to a lesser 
extent on the colliding particle. This is supported in Fig. 13. where the mean integrated jet shape is shown for different processes, viz. DIS (Breitweg et al., 1999c), $e^{+} e^{-}$collisions (Akers et al., 1994), photoproduction (Breitweg et al., 1998e) and $p \bar{p}$ collisions (Abachi et al., 1995b; Abe et al., 1993a). The results are shown for similar jet energies for all samples to remove any dependence of the particle initiator or the radiation on this quantity. In DIS and $e^{+} e^{-}$collisions the partonic system emerging from the hard interaction is expected to consist mainly of quarks. The similarity between the results from these processes, their relative narrowness and difference from the other samples, is consistent with this. The less-collimated and broader jets seen in $p \bar{p}$ collisions are indicative of gluon-initiated jets dominating the sample. In photoproduction at these energies, direct-photon processes are expected to dominate. However, the presence of QCD Compton and resolvedphoton processes also leads to gluons in the final state. The results in photoproduction lie between those from $p \bar{p}$ scattering and those from DIS and $e^{+} e^{-}$collisions, compatible with the expected mixture of gluon- and quarkinitiated jets.

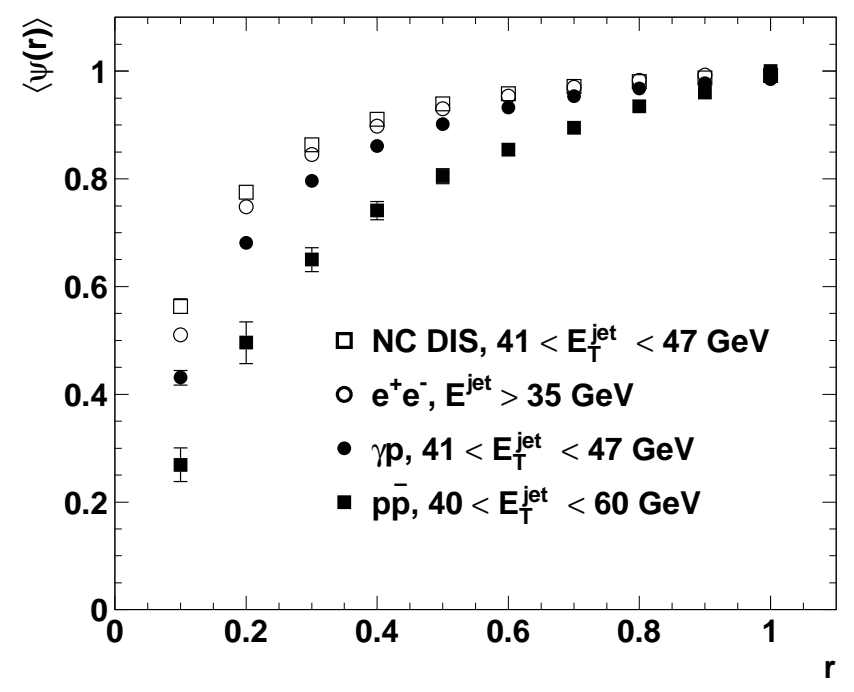

FIG. 13 Measured jet shapes in $e^{+} e^{-}$scattering (Akers et al., 1994), from ZEUS in DIS (Breitweg et al., 1999c) and photoproduction (Breitweg et al., 1998e), and in $p \bar{p}$ collisions (Abachi et al., 1995b; Abe et al., 1993a). From (Butterworth and Wing, 2005).

Measurements at HERA of internal jet structure (Adloff et al., 1999c; Breitweg et al., 1998e, 1999c; Chekanov et al., 2003d) have been compared with various Monte Carlo models and NLO QCD predictions and shown to be well described. This agreement has also allowed extractions of the strong coupling constant (Chekanov et al., 2003c, 2004i). More recently, measurements have been made (Chekanov et al., 2004j, 2009k) with the aim of distinguishing between gluonand quark-initiated jets. By cutting on the jet shape at low $r$, a jet can be classified as "broad" or "narrow" (Chekanov et al., 2004j) and thereby enriched in gluon and quark initiators, respectively.

An example dijet cross section is shown in Fig. 14, where both jets are tagged as either broad or narrow. A striking difference is observed between the two samples as a function of the cosine of the dijet scattering angle in the dijet centre-of-mass frame, with the general trends well described by the PYTHIA Monte Carlo (Siostrand, 1994; Sjostrand et al., 2006, 2001) predictions. The dijet scattering angle is a revealing quantity as it is sensitive to the propagator, with a spin $-\frac{1}{2}$ quark exchange giving a $\left(1-\left|\cos \theta^{*}\right|\right)^{-1}$ cross-section dependence and a spin-1 gluon exchange giving a $\left(1-\left|\cos \theta^{*}\right|\right)^{-2}$ crosssection dependence at leading order. The shallow rise to high $\cos \theta^{*}$ for the sample with two narrow jets and the steeper rise for the sample with two broad jets are indicative of such a difference in propagator. The angular distributions can be understood in terms of the dominant two-body processes: the resolved subprocess $q_{\gamma} g_{p} \rightarrow q g$, mediated by gluon exchange for the broad-broad dijet sample and the direct subprocess $\gamma g \rightarrow q \bar{q}$, mediated by quark exchange for the narrow-narrow dijet sample. For events with two broad jets, PYTHIA predicts the parton final state to consist of $16 \% g g, 52 \% q g$ and $32 \% q q$. For events with two narrow jets, PYTHIA predicts the parton final state to consist of $71 \% q q, 28 \% q g$ and $1 \% g g$. The relatively impure sample of gluon-initiated jets is due to the dominance of the boson-gluon fusion process (with two quark-initiated jets in the final state) and the background from $c$ and $b$ quarks, which also yield broad jets due to the longer decay chain compared to that for light quarks.

This detailed understanding of jet substructure seeded the development of the new techniques to search for the Higgs boson (Butterworth et al., 2008) or other boosted heavy particles (Abdesselam et al., 2011) which are now being used at the LHC.

\section{Prompt Photon Production}

Events containing an isolated 'prompt' (or 'direct') photon are a potentially powerful tool to study hard processes. Their main attractions lie in the insensitivity of photons to hadronisation effects and the precise energy measurements obtainable for isolated electromagnetic objects. The number of possible processes is also smaller than for the case of jet production; to leading order in both QCD and QED, prompt photon cross sections in DIS and direct photoproduction are directly sensitive to the quark content of the proton through the Compton scattering $(\gamma q \rightarrow \gamma q)$ process. Resolved-photon contributions are dominated by the $g q \rightarrow q \gamma$ process, giving sensitivity to the quark and gluon contents of both the proton and photon. These advantages have to be set against the significantly smaller event rates for prompt photon than for jet production and the experimental challenge of sep- 


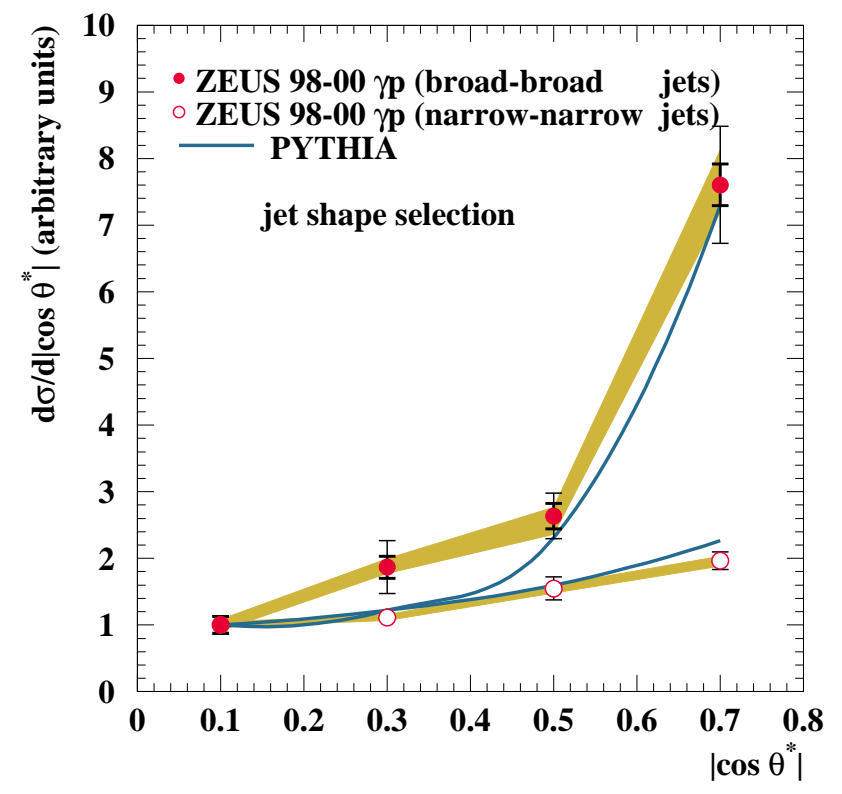

FIG. 14 Measurement of the dijet cross section differential in the cosine of the dijet scattering angle, $\mathrm{d} \sigma / \mathrm{d}\left|\cos \theta^{*}\right|$, for events with two broad jets or two narrow jets. The data are compared with PYTHIA Monte Carlo predictions. From (Chekanov et al., 2004i).

arating photon samples from backgrounds due to $\pi^{0}$ and $\eta^{0}$ meson decays to multi-photon states. This separation relies on many discriminating variables, such as the shape of the shower deposited in the calorimeter.

Measurements of prompt photon production have been made in both DIS (Aaron et al., 2008b; Abramowicz et al., 2012b; Chekanov et al., 2004e, 2010e) and photoproduction (Aaron et al., 2010i; Abramowicz et al., 2014; Aktas et al., 2005e; Breitweg et al., 1997b, 2000b; Chekanov et al., 2001b, $2007 \mathrm{~m})$. Both H1 and ZEUS have made measurements in the DIS regime using significant fractions of the available data. The data have been compared with predictions (Gehrmann-De Ridder et al., 2006a, b, 2000) to order $\alpha^{3}$, which describe the shapes of the measured cross sections, $\mathrm{d} \sigma / \mathrm{d} E_{T}^{\gamma}$ and $\mathrm{d} \sigma / \mathrm{d} \eta^{\gamma}$. However, the theory is systematically below the data, with the difference concentrated at low $Q^{2}$, as shown in Fig. 15. The alternative approach of (Martin et al., 2005) treats photons as a partonic constituent of the proton, introduced by including QED corrections to the proton PDFs and producing high energy photons in the final state. Predictions from this model are also shown in Fig. 15. They fall below the data over most of the measured range, but are close in the high- $Q^{2}$ region, where lepton emission is expected to be dominant. An improved description of the data is obtained by appropriately combining the two predictions, suggesting a need for further calculations to exploit the full potential of the measurements.

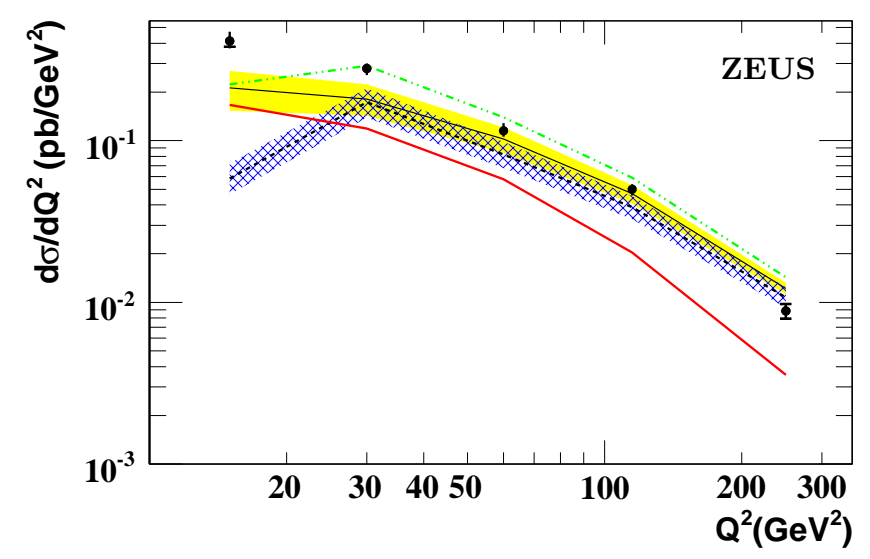

FIG. 15 Differential cross section for prompt photon production in DIS with the data compared with various theoretical predictions. An $\mathcal{O}\left(\alpha^{3}\right)$ calculation with radiation from the quark line (thick red line) and additionally including radiation from the lepton line and interference between the two (yellow shaded line) is shown. The photon is also treated by Martin et al. as a partonic constituent of the proton (blue hatched area) and including photon radiation, i.e. adding the prediction corresponding to the thick red line, from the quark line (green dot-dashed line). From (Chekanov et al., 2010e).

Cross sections for prompt photon photoproduction are shown in Fig. 16. The results use almost the full $\mathrm{H} 1$ data set and have a precision of about $10 \%$. The data are compared with an NLO QCD calculation based on collinear factorisation and DGLAP evolution (Fontannaz et al., 2001; Fontannaz and Heinrich, 2004) and with a QCD calculation based on the $k_{T}$ factorisation (Lipatov and Zotov, 2005) method which is expected to provide a good approximation for a significant part of the collinear higher-order QCD corrections. Both predictions are below the data with the largest differences at low $E_{T}^{\gamma}$ and low $\eta^{\gamma}$. The prediction based on $k_{T}$ factorisation is a bit higher than that of NLO DGLAP QCD and hence is closer to the data. The same conclusions as those stated here and seen in Fig. 16 were arrived at in the latest ZEUS results (Chekanov et al., 2007m). The influence of high-order QCD terms and hadronisation effects are expected to be largest at low transverse energies, and further theoretical developments are needed in this region in particular.

Overall, the impact of prompt photon data on the development of QCD at HERA has been somewhat disappointing compared with the successes obtained using jet observables. This is in part due to the limited statistics and experimental difficulties encountered in obtaining cross sections and partly due to the need for further improvements in the theoretical understanding to fully exploit the data. 

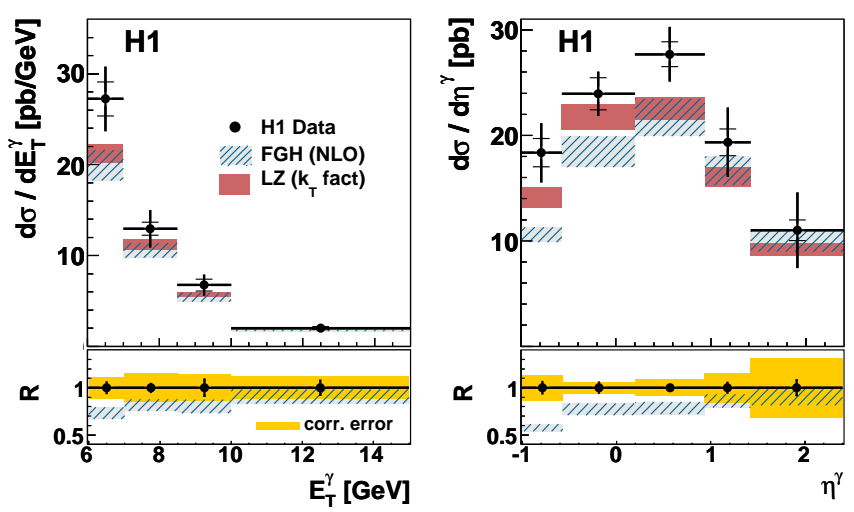

FIG. 16 Differential cross sections for prompt photons in photoproduction. The data are compared with two QCD calculations, one based on collinear factorisation in NLO (hatched histogram) and the other based on $k_{T}$ factorisation. The lower parts of the figures show the ratio of the NLO QCD prediction to the data. From (Aaron et al., 2010j).

\section{Heavy Quark Production}

Heavy quarks at HERA, as at other colliders, are tagged using many different independent techniques as summarised in Section [I] As with inclusive jet cross sections, the primary aim of measurements is to test QCD (see Eq. 3), through the sensitivity to both the perturbative prediction as discussed in this section and the proton and photon PDFs as discussed in Section III.C. As the masses of charm and particularly beauty quarks are so much larger than $\Lambda_{\mathrm{QCD}}$, they can provide a hard scale for perturbative calculations that are expected to converge rapidly. Depending on the process measured, information on the fragmentation or even decays can also be extracted and searches for excited states performed (see Section IV.C).

The description by NLO QCD of charm production in DIS is generally good, as shown in Fig. 17. The QCD prediction, "HvQDIS" (Harris and Smith, 1995a, b, 1998), is performed in the massive scheme, which means that it should be most reliable at low values of $p_{T}$. However, the theory describes the $p_{T}^{D^{*}}$ distribution up to $20 \mathrm{GeV}$ and over three orders of magnitude in the cross section. Taking the correlated theoretical uncertainties into account, there is also a good description of the $\eta^{D^{*}}$ distribution in both shape and magnitude. The good description in Fig. 17 shows that the dynamics of NLO QCD (along with the non-perturbative fragmentation inputs, given in Section IV.C can describe charm production over a wide kinematic range. Importantly, it also encourages an extrapolation using this NLO QCD prediction to the full phase-space in $p_{T}^{D^{*}}$ and $\eta^{D^{*}}$ in order to give a determination of the charm contribution, $F_{2}^{c \bar{c}}$, to the inclusive proton structure function. The validity of QCD calculations outside the measured region is unknown, but at least some confidence is given by a good description of the data within the measured region (see Section III.C).
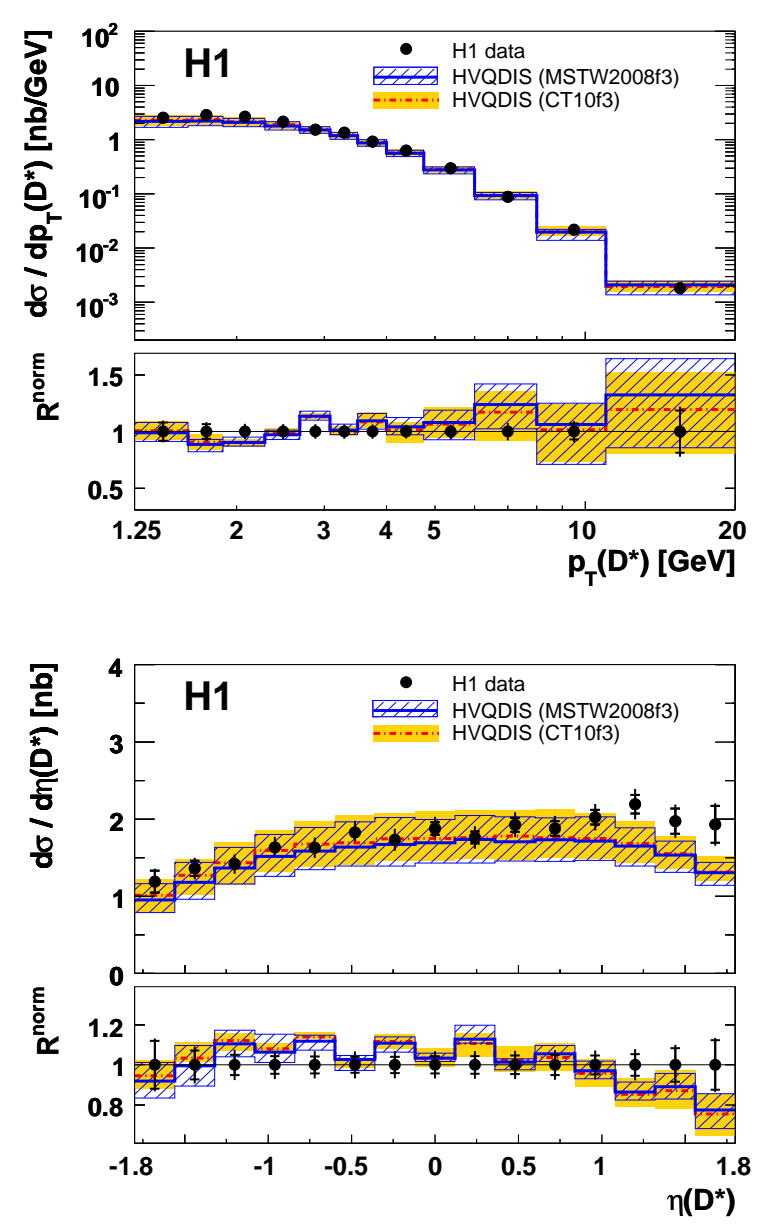

FIG. 17 Measurements of cross-sections $\mathrm{d} \sigma / \mathrm{d} p_{T}^{D^{*}}$ and $\mathrm{d} \sigma / \mathrm{d} \eta^{D^{*}}$ for $D^{*}$ production in DIS compared with NLO QCD predictions using two different proton PDFs. The lower plot for each variable shows the ratio of theory to data where each is first normalised to its corresponding total cross section. From (Aaron et al., 2011b).

Precise measurements of charm photoproduction have been made by reconstructing $D^{*}$ mesons with no explicit jet requirements Aktas et al., 2007c; Breitweg et al., $1999 \mathrm{~b})$. The details of the cross sections are not well reproduced by NLO QCD calculations, with notable problems at low values of $p_{T}^{D^{*}}$ and in reproducing the shape of the cross section as a function of the pseudorapidity of the $D^{*}$ meson. However, at the low scales measured, $p_{T}^{D^{*}} \sim 2 \mathrm{GeV}$, the uncertainties from theory are large, typically $50 \%$. More information, such as constraints on the proton and photon PDFs, could be extracted from the data with improved predictions. However, these and other such data are more commonly used to extract information on the fragmentation of heavy quarks (see Section IV.C.).

Measurements of heavy flavour photoproduction have 
also been made in which the heavy quark is part of a jet. Such measurements are generally performed at high transverse energy and, as jets are reconstructed, the measurements are usually integrated over the fragmentation fraction $z$, with correspondingly reduced sensitivity to the details of the hadronisation. Such measurements thus potentially offer a more precise comparison between data and fixed-order QCD than is the case for measurements without jet requirements. Example results are shown in Fig. 18. The descriptions of both the measured charm and beauty cross sections are reasonable, although the uncertainty on the theory is still large. Historically this level of agreement was not always the case for beauty production where at the turn of the millennium, data from the Tevatron (Abachi et al., 1995a; Abbott et al., 2000a b. c.; Abe et al., 1993b.c, 1995, 1996; Acosta et al., 2002) and the first measurement from HERA (Adloff et al., 1999e) were in strong disagreement with QCD. Through improved measurements, presenting results at the hadron rather than quark level and better theory (updated fragmentation functions and resummed calculations), this discrepancy was resolved and the need for new physics as was postulated at the time, rendered unnecessary. See (Cacciari, 2004) for a more complete discussion.
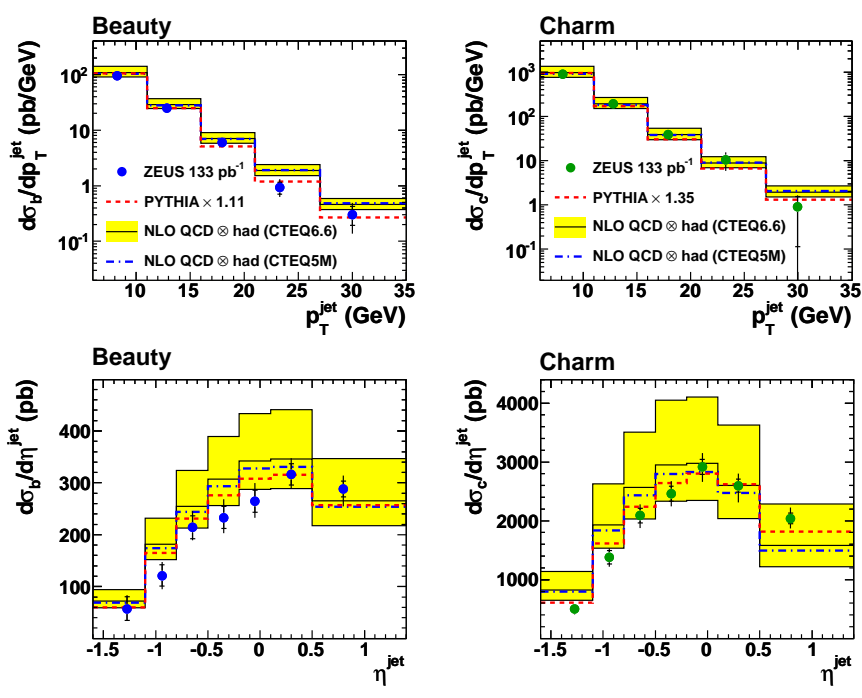

FIG. 18 Beauty (left) and charm (right) jet photoproduction cross-sections $\mathrm{d} \sigma / \mathrm{d} p_{T}^{\text {jet }}$ (upper) and $\mathrm{d} \sigma / \mathrm{d} \eta^{\text {jet }}$ (lower) compared to PyThIA Monte Carlo and NLO QCD predictions. From (Abramowicz et al., 2011b).

Since the first HERA results on beauty production, many channels covering a wide kinematic range have been measured in both DIS and photoproduction (Aaron et al., 2011a, 2012c,d, 2010i; Abramowicz et al., 2010c, 2011a; Aktas et al., 2005b.c, 2006f; Breitweg et al., 2001b; Chekanov et al., 2004a, 2007l, 2008a, 2009d, e, 2010b). The photoproduction results are summarised in Fig. 19 and compared with predictions from NLO QCD. This figure shows that beauty production at HERA is well described with particularly the most recent and most precise data in very good agreement with theory, confirming the ability of QCD to describe heavy quark production.

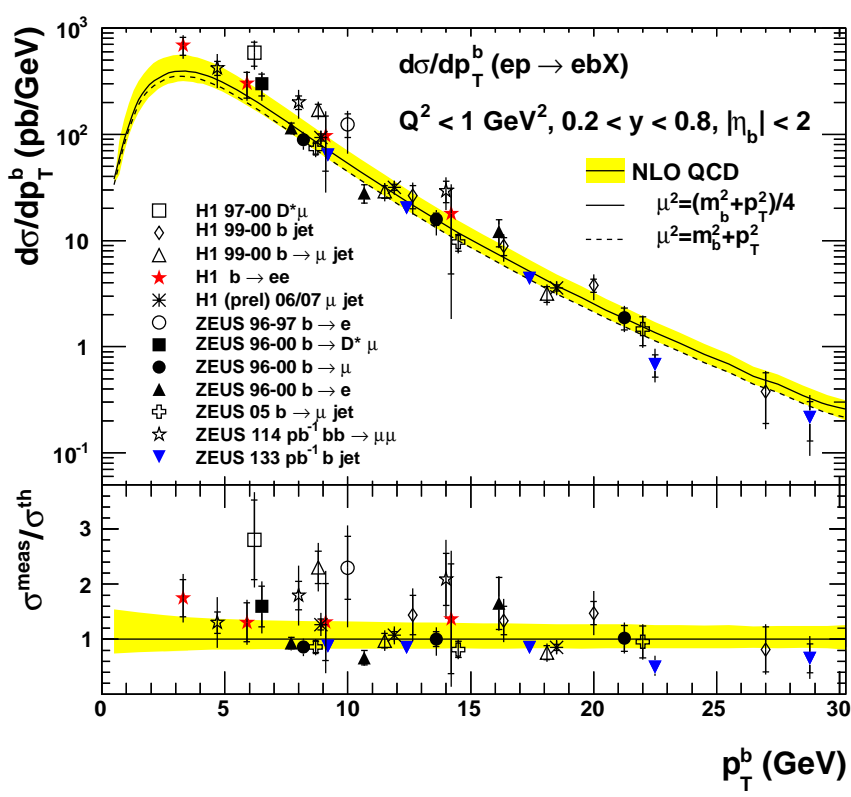

FIG. 19 Summary of beauty photoproduction measurements at HERA as a function of the transverse momentum of the $b$ quark compared with predictions from NLO QCD. The measurements, made using different final states and in different kinematic regions, were corrected for branching ratios and extrapolated to the specified kinematic region at the quark level for this comparison.

\section{Extracting Information From the Data}

As is motivated earlier in this article and can be seen from Eqs. 113, the measurements presented in this Section are sensitive to the structure of the proton and photon and to the value of the strong coupling constant. Therefore as well as comparing QCD predictions with the data, NLO QCD calculations can be used to extract the parton densities or $\alpha_{s}$.

The measurement of the structure of the proton and the extraction of its parton densities is covered in detailed reviews elsewhere (Klein and Yoshida, 2008; Perez and Rizvi, 2013), with the HERA measurements of inclusive DIS having provided the strongest constraints throughout most of the kinematic range. Final-state measurements have also been used to constrain the structure of the proton as the processes involved are directly sensitive at lowest order to the gluon distribution in the proton (see Fig. 8(a)). By using photoproduction jet data, high energy scales can also be accessed. Photoproduction jet data were included in an NLO QCD fit to ZEUS data only (Chekanov et al., 2005a) and found 
to reduce the uncertainty on the gluon distribution by a factor of two at medium to high $x(\geq 0.01)$.

The H1 and ZEUS measurements of charm production in DIS such as those in Fig. 17 have recently been combined (Abramowicz et al., 2013a), accounting for correlations in the uncertainties and thereby leading to significantly increased precision. In Fig. 20, the data are presented as a reduced cross section, $\sigma_{\text {red }}^{c \bar{c}}$, corresponding for the non-extreme $y$ values considered here to the charm contribution to the proton structure function, $F_{2}^{c \bar{c}}$. The data are compared with predictions based on parametrisations of the parton distribution functions in the proton at NLO and NNLO in QCD. These data provide extra constraints on the structure of the proton and the mechanism for charm production. In addition, the data are sensitive to the mass of the charm quark (Abramowicz et al., 2013a; Alekhin and Moch, 2011) and could be used in the context of global fits to extract this quantity. Similar measurements from both collaborations have also been made for beauty production, but with much smaller statistics.

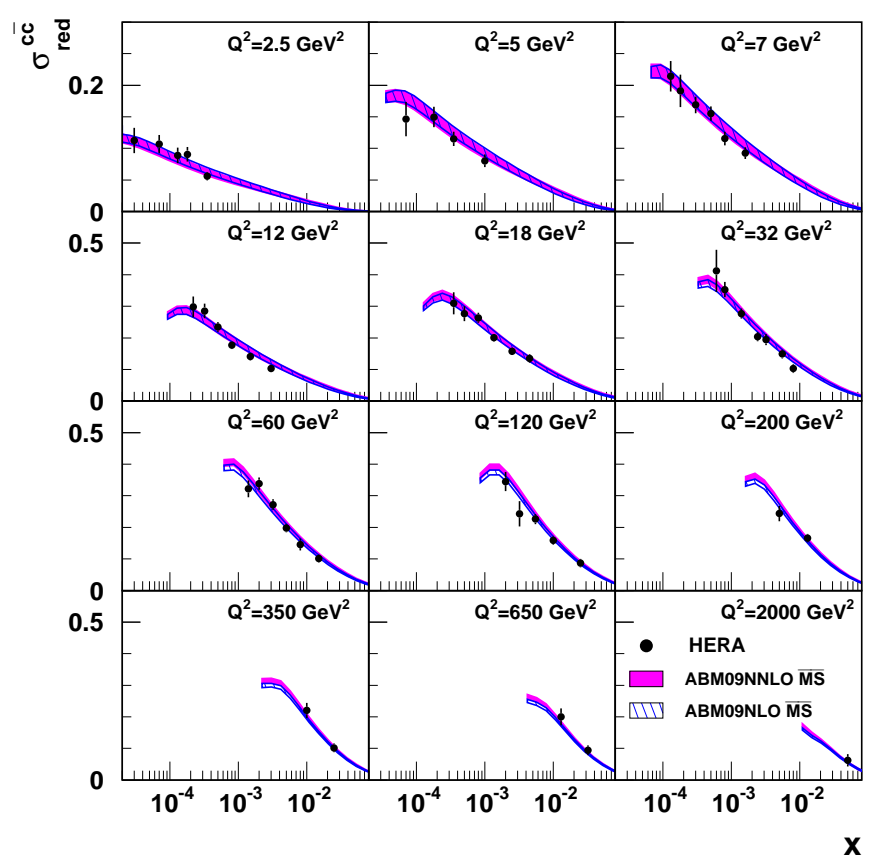

FIG. 20 Measurement of the reduced charm cross section at fixed $Q^{2}$ as a function of $x$, having combined all available published $\mathrm{H} 1$ and ZEUS data. The data are compared with a parametrisation of the proton PDF from the ABM (Alekhin et al., 2012) group at NLO and NNLO in QCD. From (Abramowicz et al., 2013a).

As can be seen in Eq. 3 and discussed in Section III.B.1. HERA data on jet photoproduction in particular are sensitive to the structure of the photon as well as the proton. The HERA data complement the measurements of $F_{2}^{\gamma}$ from $e^{+} e^{-}$colliders in that the HERA data are sensitive at LO to the gluon density in the photon, which is poorly constrained from $e^{+} e^{-}$ data. Also, the measurements from HERA probe higher scales $\left(\left\langle\bar{E}_{T}^{2}\right\rangle \sim 4000 \mathrm{GeV}^{2}\right)$ than was accessible at LEP $\left(\left\langle Q^{2}\right\rangle=780 \mathrm{GeV}^{2}\right.$ (Abbiendi et al., 2002)). There are also far more data which are sensitive to the heavy flavour structure of the photon from HERA than were obtained at LEP.

A number of challenges exist in understanding and using the HERA data in fits to the structure of the photon. To achieve as large a data sample as possible, the minimum jet transverse energy must be as small as possible. A further advantage of including the low transverse energy region is the access to correspondingly low values of $x_{\gamma}$, the region where the gluon density is expected to dominate the photon structure. However, typical cut values are at least $5 \mathrm{GeV}$, dictated by requirements in the trigger and the need to maintain a good correlation between the directions of the reconstructed jets and the partons which seed them. Furthermore, at these low values of transverse energy, the underlying event and hadronisation uncertainties prevent a precise comparison between data and QCD predictions. For example, in a measurement of inclusive-jet photoproduction by H1 (Adloff et al., 2003), the theoretical uncertainties in predicting cross sections close to the cut at $5 \mathrm{GeV}$ on the jet transverse energy, were too large to allow discrimination between different proton and photon PDFs. This measurement and the underlying event are discussed in detail in Section III.D.4. Jet cross sections can thus only be used reliably to constrain photon structure at high transverse energy, where the effects of the underlying event and hadronisation are expected to be minimised, at the expense of reduced statistics and reduced sensitivity to the gluon density at low $x_{\gamma}$. Figure 12 is an example of such a measurement where two jets are required with transverse energies above 20 and $15 \mathrm{GeV}$.

A combined fit of $e^{+} e^{-}$data and HERA dijet data has been performed in order to extract the parton densities in the photon (Slominski et al., 2006). The dijet data with high transverse energies, greater than $14 \mathrm{GeV}$, were not well described in the fit. Part of the problem is that the data are more sensitive to the gluon density in the proton than that in the photon. However, as was seen in Fig. 12, there is a significant difference between different parametrisations of the photon PDFs, suggesting that there is some flexibility which can be explored in order to achieve a better description of the data and more stringent constraints on the photon PDFs. The data also exhibit a strong need for higher order calculations (see also Fig. 23) and with such programmes, more significant constraints on the photon could be made.

The strong coupling constant, $\alpha_{s}$, has been extracted at HERA in many different processes and in a wide kinematic range, thereby providing precise results which clearly display the running with the energy scale of the process. The value of $\alpha_{s}$ has been extracted using inclusive DIS cross sections, event-shape variables (although these receive significant contributions from nonperturbative processes, see Section III.E), jet cross sec- 
tions, and ratios of rates of different processes such as the dijet cross section normalised to the total DIS cross section or the three- to two-jet rate. The ratio of the dijet to the inclusive DIS cross section is shown versus the scale, $Q^{2}$, and compared with an NLO QCD prediction in Fig. 21(a). Such a ratio reduces some systematic uncertainties which are correlated between the measurements, such as the uncertainty on the luminosity measurement. The resulting precise data are well described by the theoretical prediction. By varying $\alpha_{s}$ in the theory and fitting to the data, a value can be obtained in bins of the scale $Q^{2}$ and the running of the coupling constant with energy scale demonstrated as in Fig. 21(b). A clear variation of $\alpha_{s}$ with $Q^{2}$ is observed and the variation is well described by the two-loop solution of the renormalisation group equation.

A collection of recent determinations of $\alpha_{s}$, presented (as is conventional) at the scale of the $Z$ boson mass, is shown in Fig. 22. The two ZEUS results were extracted at high energy scales in order to minimise the theoretical uncertainties. When using the full power of the data and including the largest possible kinematic region, as for the $\mathrm{H} 1$ results, the extracted $\alpha_{s}$ values have a precision comparable to the world average (Beringer et al., 2012). However, the theoretical uncertainties, arising mainly from missing higher orders in the calculations, then become large. QCD fits to inclusive DIS data yield extractions of $\alpha_{s}$ as well as parton distribution functions. Including jet cross section information in these fits increases the sensitivity, due to the dependence on $\alpha_{s}$ at LO, and yields a precise result, as shown in Fig. 22 for the case of the 'HERAPDF1.6' fits. Again, the experimental precision of this result is competitive, but it suffers from large theoretical uncertainties. Development of higher order theoretical calculations and tools is thus now the limiting factor on the precision of strong coupling determinations from $e p$ scattering. As $\alpha_{s}$ is one of the fundamental parameters of the Standard Model and its extrapolation to very large scales provides constraints on Grand Unification, the case for prioritising such calculations is strong.

\section{Pushing the Boundaries of Applicability}

Much of this Chapter has been concerned with measurements of jet production and other hard processes in a region where the data are well modelled by NLO QCD with parton evolution governed by the DGLAP equations. This is generally the case at large transverse momenta and in a reasonably central region, where large scales are present, the strong coupling $\alpha_{s}$ is relatively small and momentum fractions $x$ are relatively large. Going beyond this region of stability requires additional theoretical tools. A successful description at smaller transverse momenta requires higher orders in the matrix elements and an improved understanding of hadronisation and underlying event phenomena. More fundamentally,
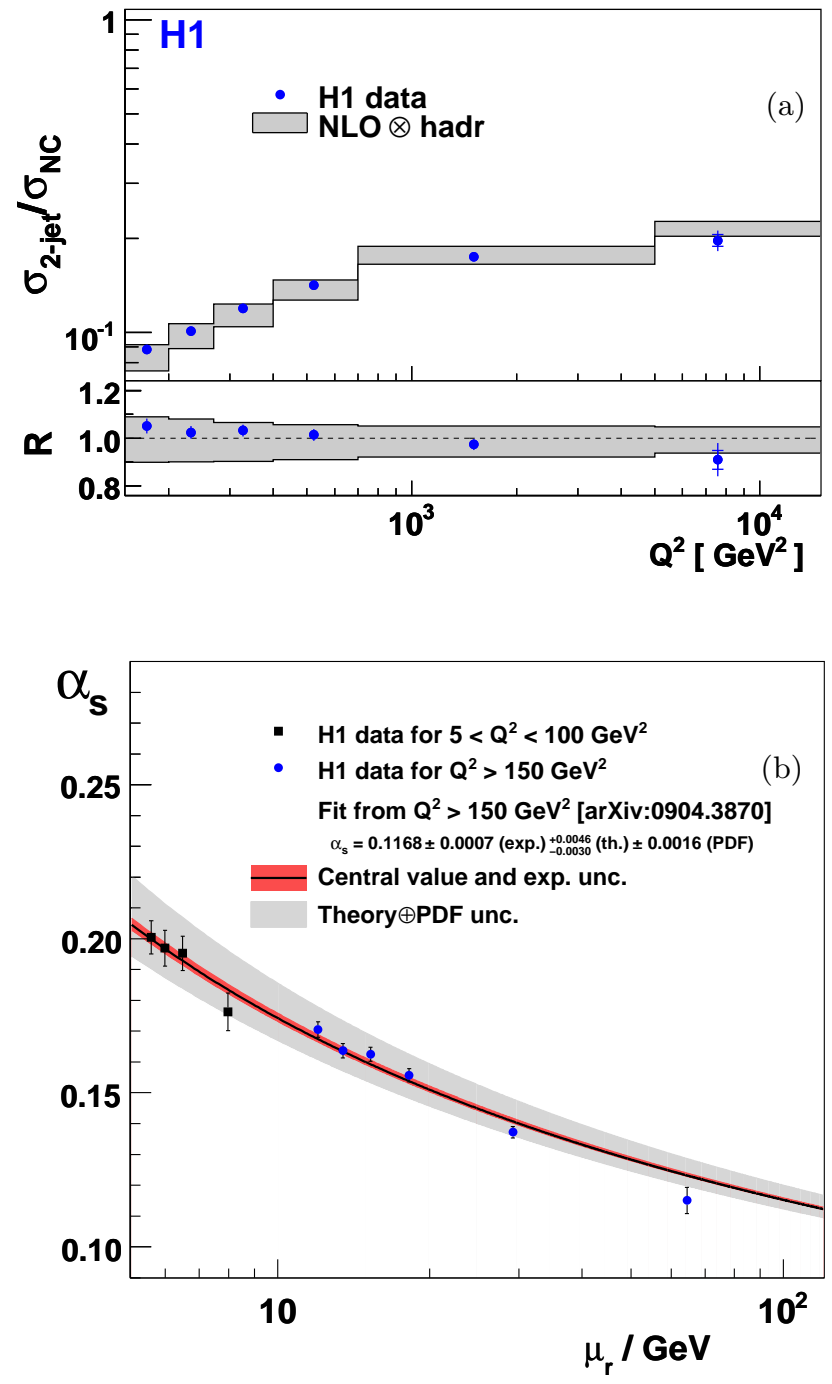

FIG. 21 (a) Measurement of the ratio of the dijet to the total cross section in DIS, compared with NLO QCD predictions (corrected for hadronisation). (b) Values of $\alpha_{s}$ versus the scale of the process extracted from distributions such as that in (a). The solid line shows the two-loop solution of the renormalisation group equation. (a) From (Aaron et al., 2010g) and (b) from (Aaron et al., 2010h).

the kinematics of the non-central region at HERA extend into a low- $x$ regime where dynamics beyond the DGLAP approximation are often considered likely to become apparent for the first time. These may consist of novel parton cascade arrangements in which the transverse momentum ordering of parton emissions between the photon and the proton intrinsic to DGLAP is broken and alternative, resummed, schemes, going beyond fixed order perturbative expansions in $\alpha_{s}$-notably the BFKL equations (Balitsky and Lipatov, 1978; Kuraev et al., 1977) are appropriate. The lack of transverse momentum ordering is intimately linked to a breakdown of collinear 


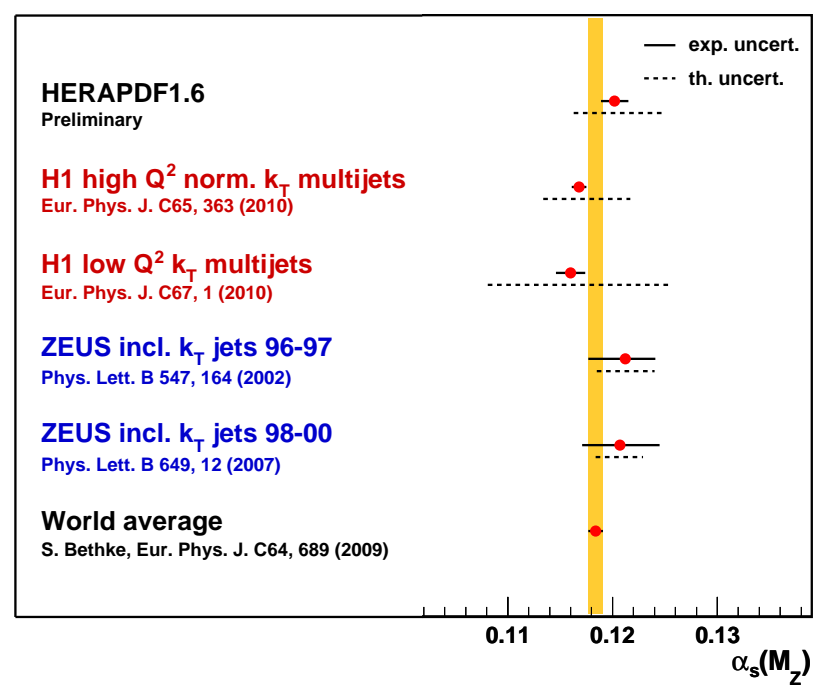

FIG. 22 Values of $\alpha_{s}$ from individual H1 and ZEUS measurements and the world average. Note the extraction in the HERAPDF1.6 fit is a 'preliminary' result.

factorisation and thus to the concept of an unintegrated gluon density of the proton, with finite and variable transverse momentum, as implemented for example in the CCFM approach (Catani et al., 1990a, b; Ciafaloni, 1988). For hard scattering processes generating transverse momenta $p_{T}>\sqrt{ } Q^{2}$ the lack of transverse momentum ordering has also been modelled by ascribing a partonic structure to the virtual photon (Gluck et al., 1996; Uematsu and Walsh, 1981). At sufficiently low $x$, phenomena associated with very high parton densities (Gribov, 1970), including non-linear evolution and parton 'saturation' may become important. Whilst such effects are not particularly relevant to the current discussion of hard processes in the inclusive final state, they have been considered in detail in the context of inclusive and diffractive DIS, where the smallest $x$ values accessed at HERA are reached (see Section $\mathrm{V}$ ).

In the following, photoproduction and DIS measurements extending beyond the best-understood region, for example to higher $\eta^{\text {jet}}$, lower $E_{T}^{\text {jet }}$ or in higher jetmultiplicity events, are summarised. In some cases, dedicated observables have been constructed which are most likely to be sensitive to novel effects beyond NLO DGLAP QCD.

\section{Higher Orders}

In this section, the inadequate description of the high$E_{T}^{\text {jet }}$ photoproduction data in Fig. 12(b) by NLO QCD is further investigated. This discrepancy could be due to a need for refined photon PDFs. However, it may also indicate that NLO QCD is insufficient and higher-order calculations are needed. A variable which is particularly sensitive to higher orders is the difference in azimuthal angle between the two jets of highest transverse energy, $\Delta \phi^{\mathrm{jj}}$. Figure 23 shows measurements of this quantity for high and low $x_{\gamma}^{\text {obs }}$, compared with NLO QCD predictions and expectations from the Monte Carlo models, HeRWIG and PYтніA, area-normalised for a comparison of shape.

For $x_{\gamma}^{\text {obs }}>0.75$, the cross section falls by about three orders of magnitude over the measured range in $\left|\Delta \phi^{\mathrm{jj}}\right|$; more steeply than for $x_{\gamma}^{\text {obs }} \leq 0.75$. At high $x_{\gamma}^{\text {obs }}$, NLO QCD agrees with the data for the back-to-back configuration (i.e. at the largest $\left|\Delta \phi^{\mathrm{jj}}\right|$ ), but it has a steeper fall off with increasing decorrelation between the jets. The prediction from the Pythia Monte Carlo programme is similar to that for NLO QCD, whereas the prediction from the HeRwig programme describes the data well. For low $x_{\gamma}^{\text {obs }}$, the NLO QCD calculation is much too steep and is significantly below the data for all values of $\left|\Delta \phi^{\mathrm{jj}}\right|$ except the highest bin. The prediction from the PyTHIA programme is less steep, but still gives a poor description. The prediction from the HeRWIG programme is in remarkable agreement with the data.
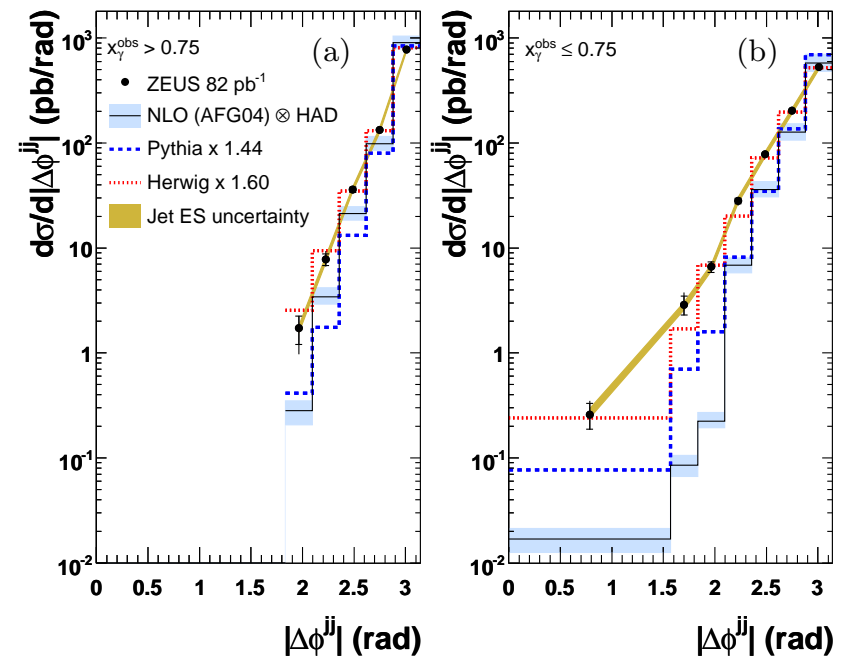

FIG. 23 Measurement of the difference in azimuth between the two highest transverse energy jets for (a) $x_{\gamma}^{\text {obs }}>0.75$ and (b) $x_{\gamma}^{\text {obs }} \leq 0.75$ compared with NLO QCD $\left(\mathcal{O}\left(\alpha \alpha_{s}^{2}\right)\right.$, corrected for hadronisation) and Monte Carlo predictions. From (Chekanov et al., 2007f).

The results here illustrate that the partonshower model in HERWIG gives a good simulation of high-order processes and suggest that matching it to NLO QCD (as is done in the programmes MC@NLO (Frixione and Webber, 2002) and Powheg (Nason, 2004), but is not yet available for these processes in $e p$ collisions) would give a good description of the data in both shape and normalisation. Should such a calculation or other high-order prediction, such as a full NNLO QCD treatment, become available, these distributions would be ideal tests, as they are inclusive 
quantities of high precision.

These results and conclusions are qualitatively similar to those for the azimuthal decorrelation in high $E_{T}$ dijet photoproduction in which at least one of the jets is tagged as originating from a charm quark (Chekanov et al., $2005 \mathrm{~b}$ ) and in the angular distribution between a jet and a prompt photon (Aaron et al., 2010j). Given that $\mathcal{O}\left(\alpha_{s}^{2}\right)$ calculations are at best LO in the $\Delta \phi$ variable, it is perhaps not surprising that the data are not always well described without NNLO QCD, or higher order, calculations.

\section{New Low $x$ Phenomena}

In contrast to the case shown in Fig. 10 and elsewhere where high- $Q^{2}$ events containing jets of high transverse energy in the central part of the detector are selected, dedicated observables are required to enhance the sensitivity to low $x$ phenomena. An example approach is to measure forward jet production cross sections in a restricted phase space, as illustrated in Fig. 24. Events containing jets in the forward direction for which the variable $x_{\text {jet }}=E^{\text {jet }} / E_{p}$ is much larger than Bjorken $x$ (denoted $x_{\mathrm{Bj}}$ here) suggest a gluon cascade which is strongly ordered in fractional longitudinal momentum, as expected for BFKL-governed evolution. Further requiring that $\left(E_{T}^{\text {jet }}\right)^{2} / Q^{2}$ is of order unity restricts evolution in transverse momentum, thus suppressing standard DGLAP evolution.

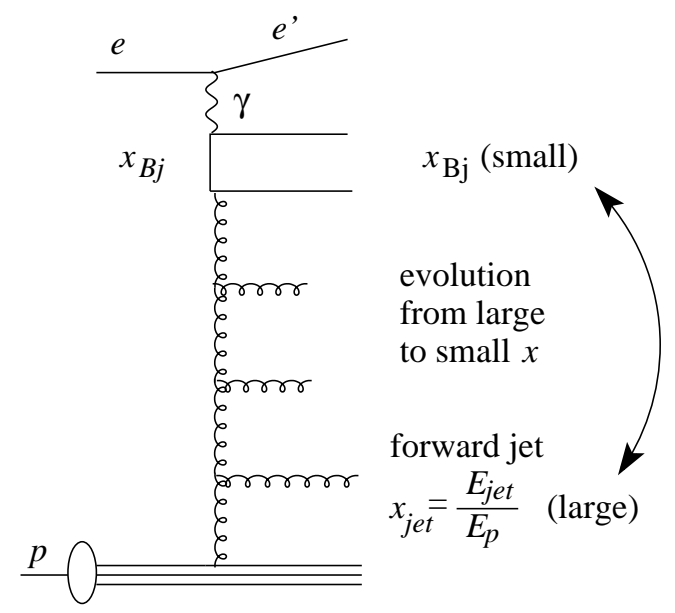

FIG. 24 Schematic illustration of $e p$ scattering with a forward jet taking a fraction $x_{\text {jet }}$ of the proton momentum. The evolution in the longitudinal momentum fraction, $x$, from large $x_{\text {jet }}$ to small $x_{\mathrm{Bj}}$ is indicated. From (Aktas et al., 2006d).

An example result using this technique is shown in Fig. 25 (Aktas et al., 2006d). The events selected here are required to have low $Q^{2}$ and low $x_{\mathrm{Bj}}$, specifically $5<Q^{2}<85 \mathrm{GeV}^{2}$ and $0.0001<x_{\mathrm{Bj}}<0.004$, and also to contain jets of transverse energy $E_{T}^{\text {jet }}>3.5 \mathrm{GeV}$ in the laboratory frame in the forward part of the detector, $7^{\circ}<\theta^{\text {jet }}<20^{\circ}$. The $x_{\text {jet }}$ variable is required to be larger than 0.035 and the scale evolution is restricted via $0.5<\left(E_{T}^{\text {jet }}\right)^{2} / Q^{2}<5$. The same data are displayed in each of the three sub-figures, but they are compared with different theoretical calculations and models.
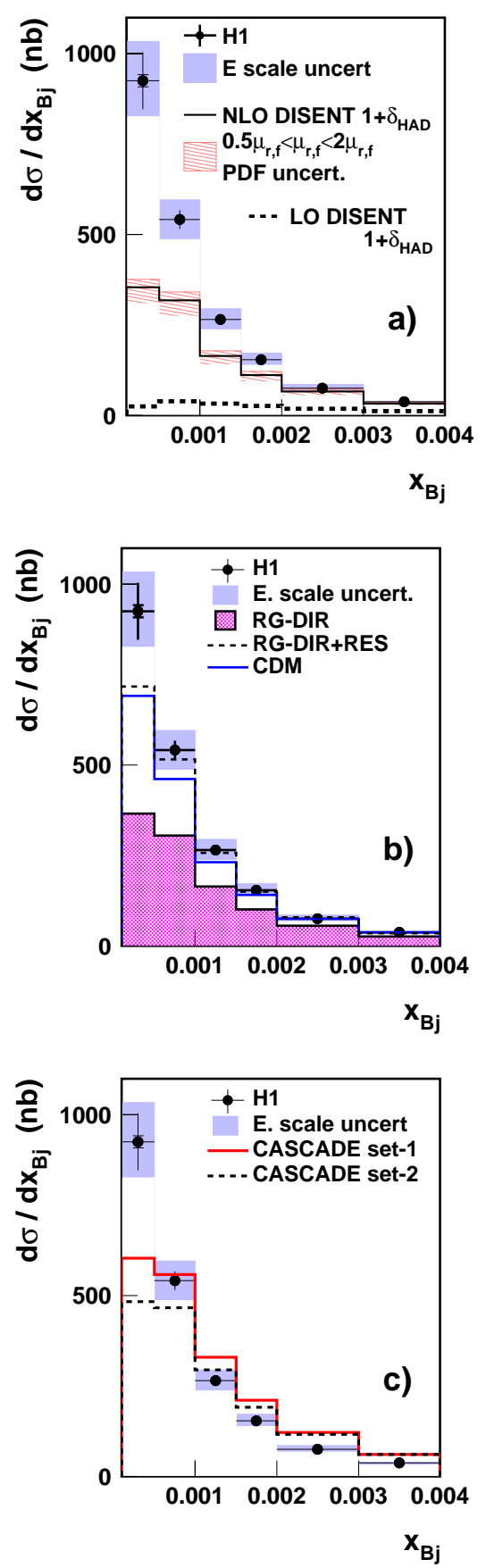

FIG. 25 Measurement of forward jet production at low $x_{\mathrm{Bj}}$ in DIS compared with (a) NLO DGLAP QCD (corrected for hadronisation) and (b) and (c) Monte Carlo models. From (Aktas et al., 2006d).

In Fig. 25(a), the data are compared with LO and NLO 
QCD, based on DGLAP evolution, i.e. the same calculations which described the data well in Fig. 10. The prediction from LO QCD is significantly below the data, as expected for the analysis phase space, which suppress the LO contribution. The NLO QCD prediction is in agreement with the data at high $x_{\mathrm{Bj}}$, but increasingly deviates from the data with decreasing $x_{\mathrm{Bj}}$. Notably, at low $x_{\mathrm{Bj}}$ the NLO QCD prediction is over a factor of 10 higher than the LO QCD prediction, suggesting that the remaining factor of two difference between data and NLO QCD may be resolved by the inclusion of NNLO QCD, assuming that the perturbative series is quickly convergent. The scale uncertainty band, obtained by the conventional method of varying the renormalisation scale by a factor of two, is at the $10 \%$ level. However, the order of magnitude difference between the LO and NLO QCD predictions suggests that this may not be fully representative of the uncertainties due to missing higher orders.

To ascertain whether predictions based on BFKL or otherwise-modified parton evolution provide a better description than DGLAP-based models, these results would ideally be compared with QCD calculations of nextto-leading-logarithmic (NLL) accuracy, summing terms in either $\ln \left(Q^{2}\right)$ or $\ln (1 / x)$. Unfortunately, such calculations are not yet available and in their absence, Monte Carlo models which incorporate appropriate LO matrix elements and leading-logarithmic parton showers are usually used. The RAPGAP model (Jung, 1995) uses LO matrix elements and parton showers based on standard DGLAP evolution. As shown in Fig. 25(b) this prediction (labelled 'RG-DIR') lies below the data most significantly at low $x_{\mathrm{Bj}}$. The inclusion of a resolved virtual photon contribution (RG-DIR+RES, see next section) significantly improves the description of the data. The colour dipole model (labelled 'CDM' in the figure) (Andersson et al., 1989; Lonnblad, 1995), uses LO matrix elements with parton emissions generated by spanning colour dipoles between the partons in place of the usual leading-logarithmic transverse momentumordered parton showers. As the dipoles in the CDM radiate independently, there is no ordering of the emissions in transverse momentum and hence this approach shares a similar characteristic with BFKL evolution. The description of the data by this model is considerably better than that by RG-DIR. Given the large point-to-point correlated uncertainties in the data and the unquantified uncertainties in the predictions, the CDM model is in fair agreement with the data.

An alternative model which incorporates BFKLlike characteristics is CASCADE (Jung, 2002; Jung and Salam, 2001). This model in fact uses the CCFM equation (Catani et al., 1990a) b; Ciafaloni, 1988; Marchesini, 1995), which provides a bridge between the DGLAP and BFKL descriptions by resumming both $\ln \left(Q^{2}\right)$ and $\ln (1 / x)$ terms, resulting in ordering by emission angle in the parton cascade. Comparisons of the CASCADE model with the data are shown in Fig. 25(c) for two different parametrisations of the unintegrated gluon density of the proton. The comparison with data is again better than that of NLO DGLAP QCD or RG-DIR at low $x_{\mathrm{Bj}}$, though it still falls short of the measured cross section at the lowest value and there are also substantial discrepancies at high $x_{\mathrm{Bj}}$.

Further measurements have been made of related observables to that shown in Fig. 25] A similar analysis has been performed (Aaron et al., 2008d) of events which contain a jet in the central region of the detector and two jets in the forward region. The data are shown as a function of the Bjorken scaling variable, $x$, in Fig. 26 and are compared with QCD calculations to different orders in $\alpha_{s}$. The curves labelled $\mathcal{O}\left(\alpha_{s}^{2}\right)$ and $\mathcal{O}\left(\alpha_{s}^{3}\right)$ represent LO and NLO QCD predictions for this three-jet process, respectively. Therefore, again due to kinematic restrictions, it is not surprising that the LO prediction fails to describe the data. The NLO prediction is considerably better, but still lies below the data at the lowest $x$, indicative of the need for higher-order calculations or dynamics beyond the DGLAP approximation.

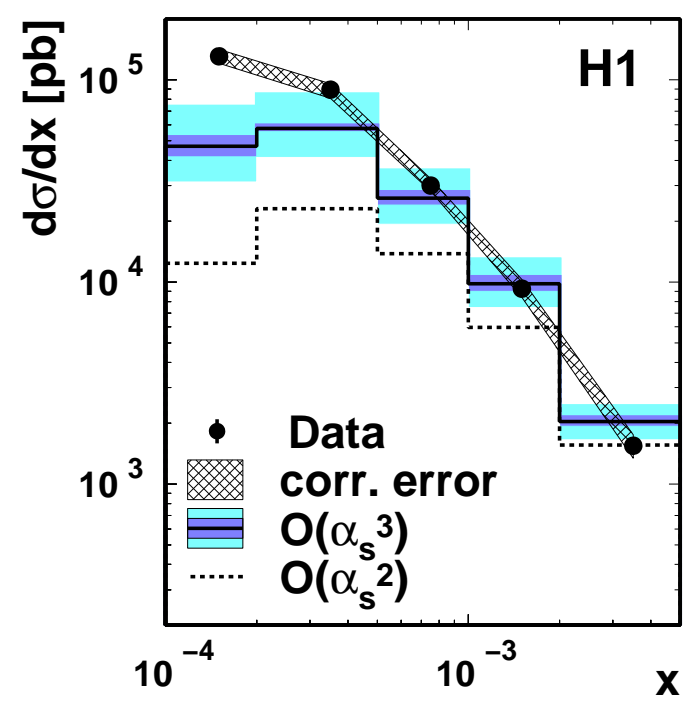

FIG. 26 Measurement of the cross section as a function of the Bjorken scaling variable, $x$, for events in which two jets are reconstructed in the forward direction and one central. The data are compared with second- and third-order QCD calculations. From (Aaron et al., 2008d).

Other related measurements include replacing the forward jets by forward $\pi^{0}$ mesons (Adloff et al., 1999b; Aktas et al., 2004b), which is complementary in that it probes lower values of $x_{\mathrm{Bj}}$, but at the expense of reduced signal purity. Measurements of azimuthal decorrelations between jets at low $x$ (Aktas et al., 2004c) and between a forward jet and the scattered lepton (Aaron et al., 2012f) have also been made. As in the examples above, common themes in these analyses include tendencies for NLO DGLAP QCD predictions to lie below the data at the lowest $x$ and for the CDM model to provide improved descriptions. 


\section{Virtual Photon Structure}

As discussed in Section II.F the distinction between DIS and photoproduction is somewhat arbitrary. Although no component due to the structure of the photon is usually included in calculations of DIS processes, such a component may still be relevant where an event contains a hard process with a scale such as a jet transverse momentum which is larger than that provided by the virtuality of the photon. Under these circumstances, the photon may be considered as being the object whose structure is being probed.

A measurement which is sensitive to the need for a resolved virtual photon contribution is shown in Fig. 27. The cross section for dijet production in DIS is shown as a function of $x_{\gamma}^{\text {obs }}$ (see Eq. 4), here called $x_{\gamma}^{\text {jets }}$. The data (Aktas et al., 2004e) are shown for different regions of $Q^{2}$ and jet transverse energy $E_{T}^{*}$ in the $\gamma^{*} p$ frame. Comparisons are made with various QCD calculations. The predictions from DISENT and 'NlOJET for 2 jets' are both NLO QCD predictions for dijet production in DIS (i.e. $\left.\mathcal{O}\left(\alpha_{s}^{2}\right)\right)$. Both of these calculations successfully describe high $Q^{2}$ data, but for this variable at low $Q^{2}$ and low $E_{T}^{*}$, they lie significantly below the data at low $x_{\gamma}^{\text {jets }}$. The other calculation shown, 'JETVIP', should in principle be ideal to describe these data as it has a component due to the resolved virtual photon which may be inferred from the difference between the 'full' and 'dir' predictions. Although it can be seen that the addition of this component brings the NLO QCD calculations significantly closer to the data at low $x_{\gamma}^{\text {jets }}$, the 'dir' component does not agree with DisENT or NLOJET at the level that they agree with each other, though all three are in principle calculations of the same contribution. Due to this, the JETVIP calculations cannot be considered to be fully reliable. It is unlikely that these calculations will be improved and hence firm conclusions on the influence of virtual photon structure on the description of observables such as this in NLO QCD cannot yet be drawn. Alternative predictions have been made (Chyla et al., 2005) using the NLOJET calculation in its mode for calculating three-jet production ('NLOJET for 3 jets'). Although this is not a full NNLO QCD $\left(\mathcal{O}\left(\alpha_{s}^{3}\right)\right)$ calculation for dijet production, as an NLO QCD calculation for $2 \rightarrow 3$ processes, it contains a number of the extra diagrams. The prediction lies significantly higher than that for 2 jets, particularly at low $x_{\gamma}^{\text {jets }}$, and the description of the data is significantly improved.

\section{The Underlying Event}

The term 'underlying event' is usually used in high transverse momentum processes to refer to all hadronic final state particles not originating from the hard partonic scattering. Whilst in principle this includes the influence of the proton, and possibly the photon, beam remnants, the most interesting component arises from the

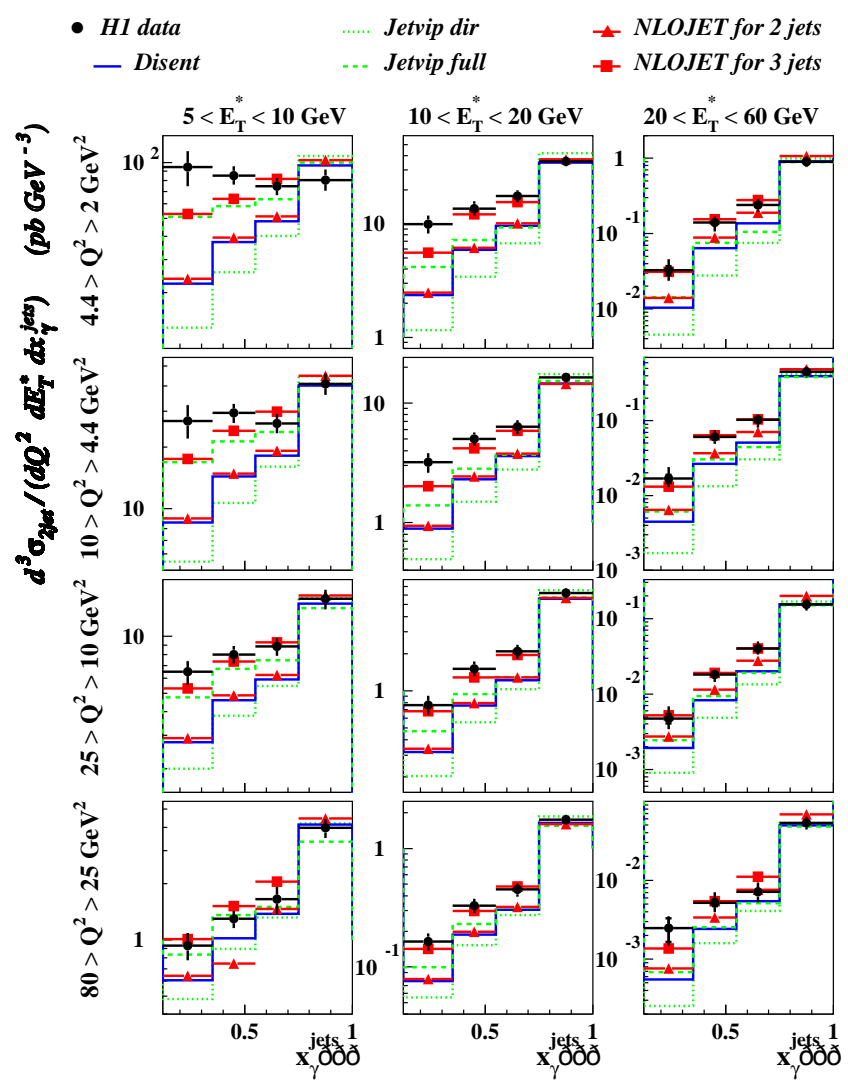

FIG. 27 Measurement of the dijet cross section in the $\gamma^{*} p$ frame in DIS as a function of $x_{\gamma}^{\text {jets }}$ in different regions of $Q^{2}$ and $E_{T}^{*}$. The data (Aktas et al., 2004e) are compared with three NLO QCD calculations in DIS for $2 \rightarrow 2$ processes, 'Disent', 'Jetvip dir' and 'Nlojet for 2 jets', with an NLO QCD calculation for $2 \rightarrow 3$ processes, 'NLOJET for 3 jets', and with an NLO QCD calculation in DIS for $2 \rightarrow 2$ processes, including a component of resolved virtual photon structure, 'JETVIP full'. All of the theory predictions are corrected for hadronisation effects. From (Chyla et al., 2005).

possibility of multiple photon-proton scatterings taking place in the same event. As with photon structure, the underlying event is therefore a concept most naturally applied to resolved photoproduction, but which in principle may still be relevant to DIS when the photon virtuality is not significantly larger than other hard scales in a process. The secondary scatters generate additional hadronic energy flow in the event, the topology and magnitude of which are poorly understood theoretically, but which must be accounted for when measuring jet and other related cross sections. As illustrated in Fig. 28, jet measurements are most sensitive to underlying event issues at relatively low transverse momentum, where they may significantly influence the shapes as well as the normalisations of distributions.

At the centre of the development of our understanding of the underlying event is the possibility of multiple hard interactions (Landshoff and Polkinghorne, 1978), 


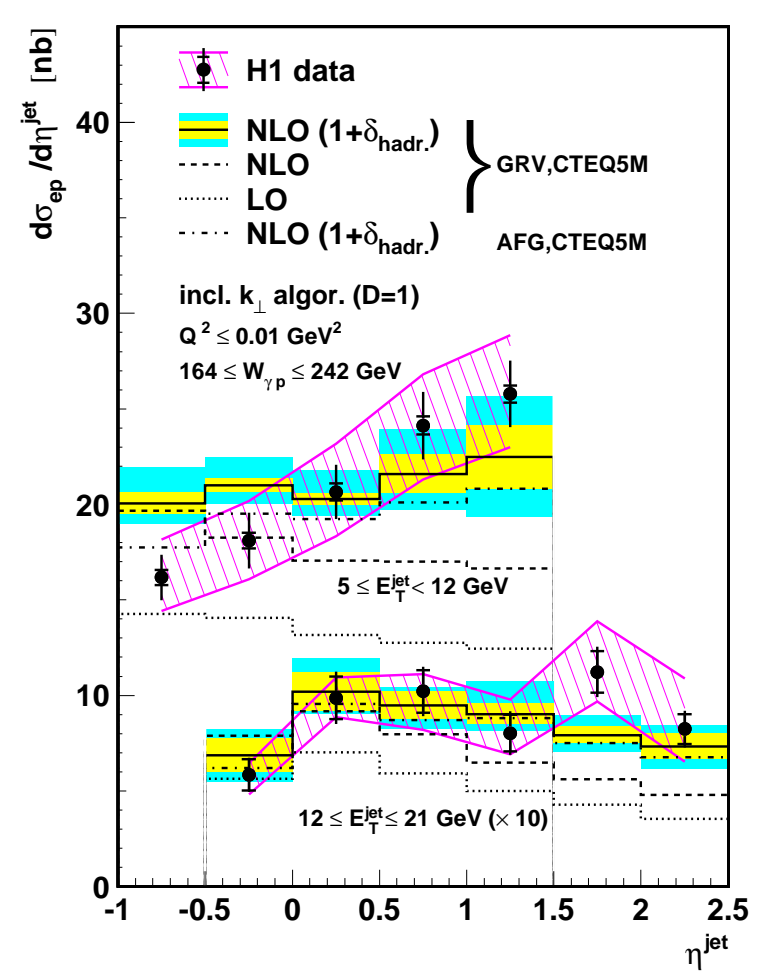

FIG. 28 Measurements of inclusive jet photoproduction at the smallest jet transverse energies reached at HERA (extending to $E_{T}^{\text {jet }}=5 \mathrm{GeV}$ ). The data are compared with NLO QCD calculations before and after correcting for underlying event and hadronisation effects, the combination of which is labelled $\delta_{\text {hadr. }}$. From (Adloff et al., 2003).

in which more than one pair of partons takes part in separate processes that generate large transverse momenta (see Fig. 291). Such processes are usually modelled (Butterworth et al., 1996; Sjostrand et al., 2001) via an integral over different impact parameters between the two hadrons, for each of which secondary partonic scattering is generated using proton PDFs at a suitably reduced momentum fraction, distributed over an appropriate spatial matter distribution. A consequence of this impact parameter-dependent physical picture is that the hardest primary interactions are associated with the most 'head-on' of collisions and are thus predicted also to exhibit high transverse momentum secondary scatterings. This predicted correlation encourages an experimental approach based on events with high jet multiplicities.

Multi-parton interactions have been studied at the Tevatron (Abazov et al., 2003; Abe et al., 1993d, 1997a b; Acosta et al., 2004), in dijet photoproduction events at HERA (Adloff et al., 2000c; Aid et al., 1996d; Breitweg et al., 1998b; Derrick et al., 1995a) and more recently at the LHC (Aad et al., 2011b c, 2012c; Chatrchvan et al., 2011, 2012b, 2013b; Khachatryan et al., 2010). In the case of the HERA data, the inclusion of multi-parton interactions in

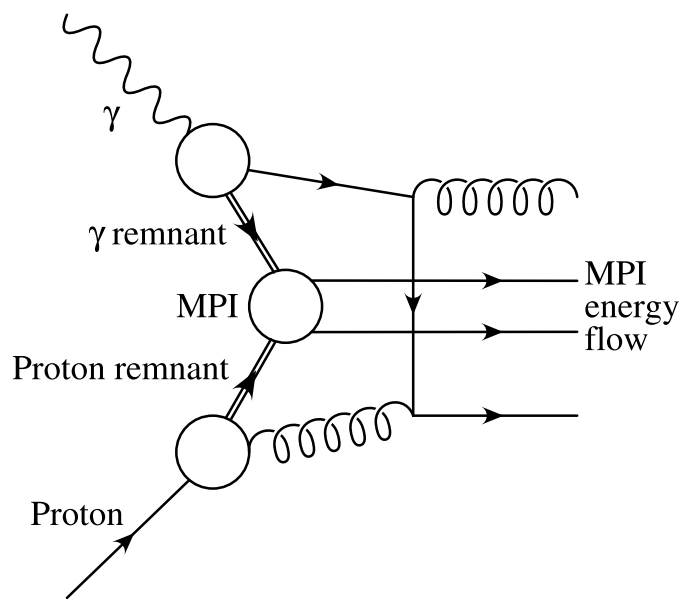

FIG. 29 A schematic representation of an event with multiparton interactions.

Monte Carlo models improves the description of the data at low transverse energy for regions enriched in resolved-photon events, as expected. Supportive of the underlying event models though this is, it does not in itself constitute evidence for the existence of hard secondary scatters. To search for direct evidence of such processes, photoproduction events containing four jets with $E_{T}^{\text {jet }}>6 \mathrm{GeV}$ have been considered. Events produced via multi-parton scattering exhibiting this topology are in principle distinguishable from four-jet events produced via QCD radiation in ordinary $2 \rightarrow 2$ scattering through the lack of correlation between the angular distributions or momenta of the pairs of jets. The measured cross section for four-jet photoproduction is shown as a function of $x_{\gamma}^{\text {obs }}$ (Chekanov et al., 2008f) in Fig. 30, compared with various predictions from Monte Carlo models. As with the case of dijet production, but even more significantly, adding models of multi-parton interactions to the predictions significantly improves the description of the data, with models not including this feature being clearly inadequate. However, investigation of angular correlations in these data did not reveal clear evidence of independent secondary scatters.

In summary, although some HERA data show clear evidence for some form of underlying event, the exact nature of the effect is not yet firmly established. Models which are based on multiple independent hard secondary scatters significantly improve the description of the data, but no direct evidence for this process has yet been demonstrated. In any case, the HERA data will help to constrain future underlying event models and Monte Carlo tunes.

\section{Discussion}

The above considerations clearly indicate that an improved description of HERA hadronic final state data 


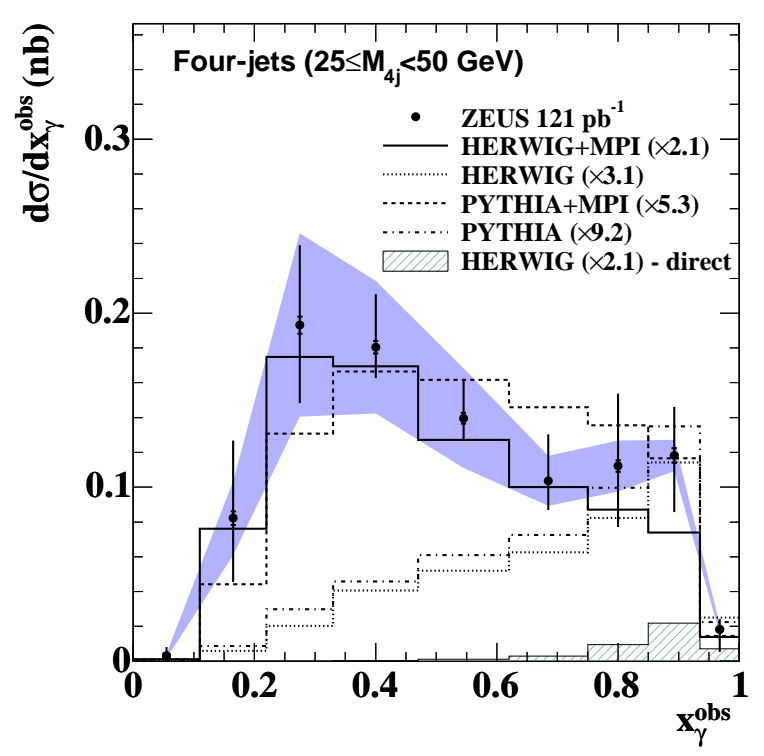

FIG. 30 Measurement of the four-jet photoproduction cross section as a function of $x_{\gamma}^{\text {obs }}$ for low invariant masses of the four-jet system, $25<M_{4 \mathrm{j}}<50 \mathrm{GeV}$. The predictions from the HeRwIG and PyTHIA MC models are shown both with (Butterworth et al., 1996; Siostrand et al., 2001) and without multi-parton interactions, as is the directphoton component of the HERWIG prediction. Each prediction is area-scaled to the measured high-mass $\left(M_{4 \mathrm{j}}>\right.$ $50 \mathrm{GeV}$ ) cross section by the factors given in the legend. From (Chekanov et al., 2008f).

could be obtained with higher-order (either NNLO QCD or NLO QCD with parton showering) calculations. Tools providing such calculations for comparisons with LHC data have generally been successful for similar observables to those considered here (see e.g. Aad et al., 2012a)). All further statements beyond this central conclusion are less definite. There is evidence for novel parton cascade dynamics, similar to those expected for BFKL-dominated evolution, in low $x$ HERA hadronic final state data. However, the extent to which such effects can be recovered through higher order DGLAP-based calculations is not yet clear. In the absence of such calculations, models based on virtual photon structure are often as successful in simulating the effects as those invoking alternative parton evolution schemes. The data also show considerable circumstantial evidence for multi-parton interactions. However, in the absence of a direct observation of such processes, it is not yet possible to rule out the possibility that the apparent need for such effects is a consequence of missing higher-order QCD calculations.

\section{E. Event Shapes}

The hadronic final states of events in DIS and $e^{+} e^{-}$ annihilation can be characterised by a number of vari- ables that describe the shape of the event. These variables are sensitive to perturbative and non-perturbative QCD and given these are properties which are measurable for all events, the data samples are much larger than can be obtained for example by requiring high$E_{T}$ jets in DIS. Understanding the hadronisation process is crucial to all measurements in this article and is often modelled phenomenologically using Monte Carlo event generators and applying the resulting corrections to fixed-order QCD calculations. An alternative approach is to use power corrections, which have an $\mathcal{O}(1 / Q)$ dependence, and are calculated analytically, extending perturbative methods into the non-perturbative regime. Within this framework (Dokshitzer et al., 1998, 1999, 1996; Dokshitzer and Webber, 1995, 1997), event-shape variables depend on the strong coupling, $\alpha_{s}$, and an effective coupling parameter, $\alpha_{0}$, which is expected to be universal for all event shapes.

Event shapes have been measured in $e^{+} e^{-}$annihilation (for a recent review and the citations therein, see (Okorokov, 2012)) and power corrections were found to reproduce many aspects of the hadronisation process. The measurements discussed here from H1 (Aktas et al., 2006g) and ZEUS (Chekanov et al., 2007d) use DIS events reconstructed in the Breit frame with a minimum $Q^{2}$ of 196 and $80 \mathrm{GeV}^{2}$, respectively. These most recent measurements have significantly larger data samples than previous publications (Adloff et al., 1997f, 2000b; Breitweg et al., 1998d; Chekanov et al., 2003a) and therefore allow measurement of differential eventshape distributions rather than just the mean values. The event shapes studied are: the thrust, which measures the longitudinal collimation of the hadronic system relative to an appropriate axis; the broadening, which measures the complementary aspect; the jet mass; and a characteristic of the event known as the $C$-parameter (for the exact definitions, the reader is referred to the H1 and ZEUS publications and their references.) An example of the measurements is shown in Fig. 31, where the mean event shapes are compared with a prediction using NLO QCD to describe the perturbative production of partons and power corrections to describe the hadronisation process. The figure also shows the prediction from NLO QCD alone, indicating the strong need for power corrections, which, when combined with the NLO QCD prediction, describe the data well. The differential distributions of all variables are similarly well described by the theory if the perturbative part is a combination of the NLO QCD prediction matched to a resummed prediction to NLL accuracy.

The good description of the differential distributions and their mean values allow the parameters of the theory, $\alpha_{s}$ and $\alpha_{0}$, to be extracted from the data. The extracted values from fits to the differential distributions are shown in Fig. 32 in the plane of the two extracted variables for all five event shapes from both collaborations. In the case of H1, Fig. 32(a), the values of $\alpha_{s}$ extracted for the five variables agree reasonably well with each other and with 

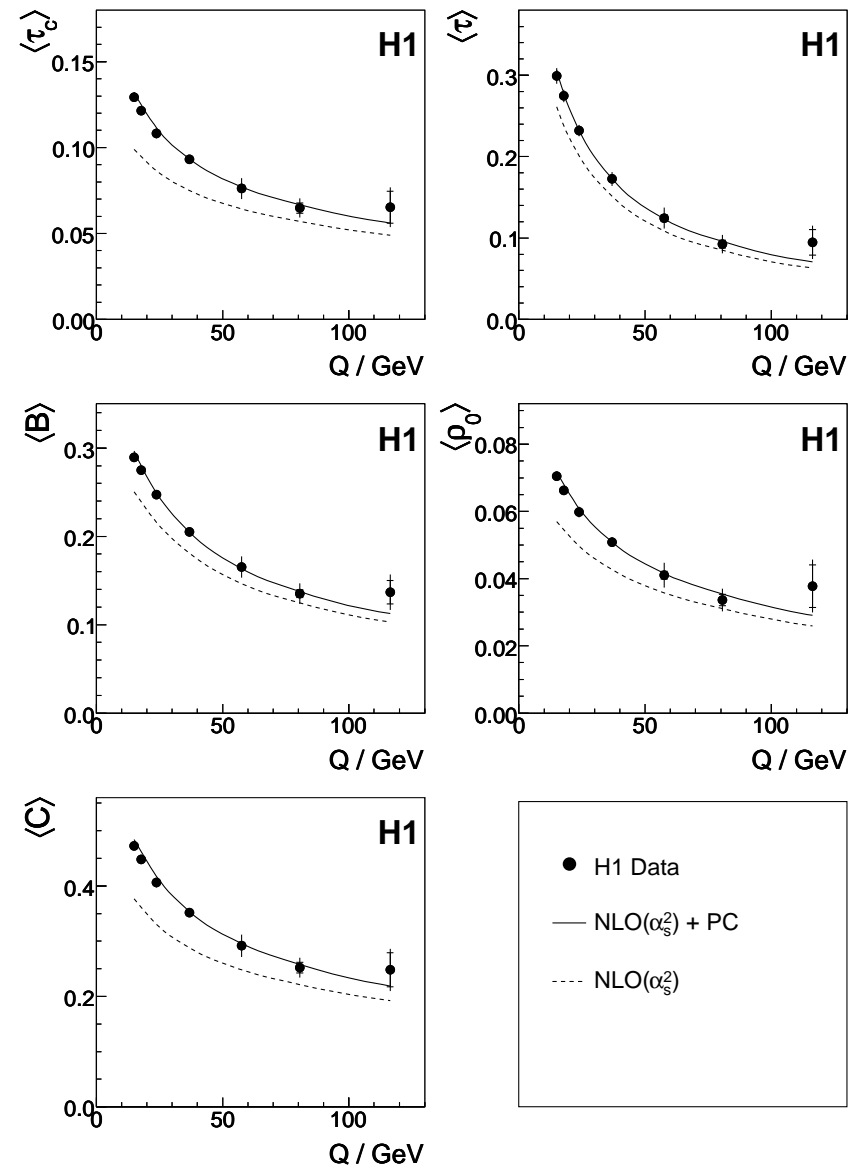

FIG. 31 Distributions of mean event shapes: the thrust variable, $\tau$, which measures the longitudinal momentum components projected onto the boson axis; the thrust variable, $\tau_{C}$, which maximises the sum of the longitudinal momenta in the current hemisphere; the broadening variable, $B$, which measures the scalar sum of transverse momenta with respect to the boson axis; the squared jet mass, $\rho_{0}$, normalised to four times the squared scalar momentum sum in the current hemisphere; and the $C$-parameter. The data, as a function of the scale, $Q$, are compared with the result of a fit based on NLO QCD with power corrections (solid line) as well as the contribution from NLO QCD alone (dashed line.) From (Aktas et al., 2006g).

the world average. H1 also investigated the dependence of $\alpha_{s}$ on the scale, $Q$, and observed the expected variation predicted by the renormalisation group equation. Similarly, the values of $\alpha_{0}$ are all broadly consistent with each other. Averages of $\alpha_{s}$ and $\alpha_{0}$ were then extracted as $\alpha_{s}\left(M_{Z}\right)=0.1198 \pm 0.0013(\text { exp. })_{-0.0043}^{+0.0056}$ (theo.) and $\alpha_{0}=0.476 \pm 0.008$ (exp. $)_{-0.059}^{+0.018}$ (theo.). A similar analysis from ZEUS is shown in Fig. 32(b), where the values of $\alpha_{s}$ extracted also agree with each other and the world average. The values of $\alpha_{0}$ are similar but show a larger spread, with the $C$-parameter showing the largest difference from the results of H1. It should be noted that: the uncertainty due to variation of the renormalisation scale
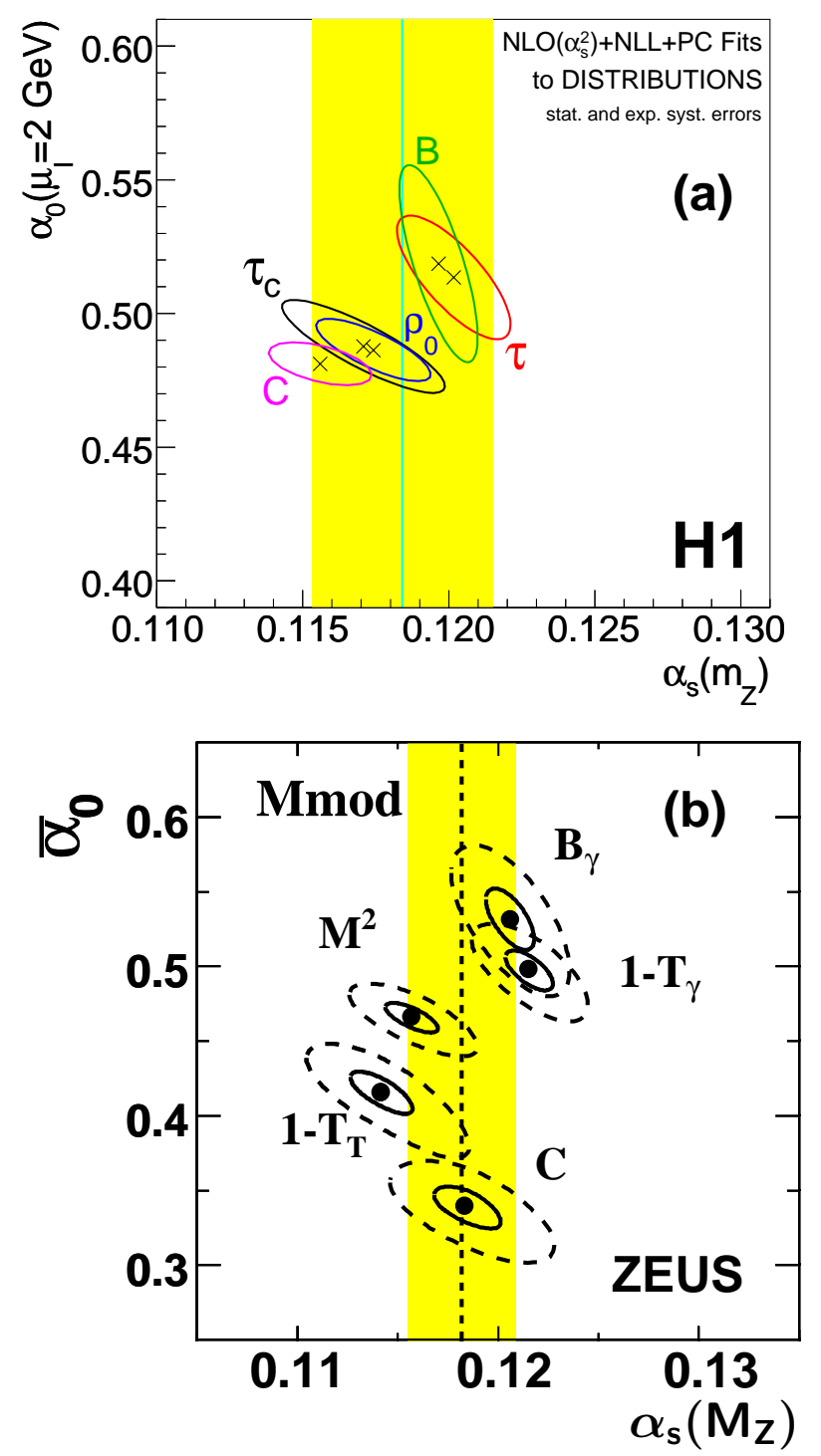

FIG. 32 Extracted values from (a) H1 and (b) ZEUS for $\alpha_{0}$ and $\alpha_{s}$ for fits of the NLO+NLL+PC predictions to all eventshape variables. The solid lines represent the $1 \sigma$ contours and for (b) the dashed line represents the $95 \%$ C.L. contour. The world-average value of $\alpha_{s}$ and its uncertainty are shown as the bands, taken from (Bethke, 2004). (a) From (Aktas et al., 2006g) and (b) from (Chekanov et al., 2007d).

is significantly larger than the experimental uncertainty; the extraction of the parameters is sensitive to the kinematic range chosen for the fits; and the parameters are also sensitive to the details of the matching performed to combine the NLO and NLL QCD predictions. These issues point to a need for higher-order calculations and in future a unified approach to fitting the data, both from DIS and $e^{+} e^{-}$annihilation.

In summary, the general description of the event-shape distributions and mean values at HERA is good, showing 
the applicability of $\mathrm{pQCD}$, however with indications, as in the rest of this chapter, that orders beyond NLO QCD are needed. The combination of power corrections, to describe the hadronisation process, with pQCD is also supported, although this can depend on the precise details of the analysis. Other aspects and models of hadronisation are discussed in the next chapter.

\section{NON-PERTURBATIVE ASPECTS AND HADRONISATION}

In this section, measurements of hadron production and their interpretations in QCD, both perturbative and non-perturbative, are discussed. Charged particle distributions and Bose-Einstein correlations are first discussed and comparisons are made with $e^{+} e^{-}$and other data to test the universality of fragmentation. The parameters of charm fragmentation are then presented and again comparisons are made between different reactions. Inelastic $J / \psi$ results follow, together with a discussion of HERA's contribution to the understanding of charmonium production mechanisms. Finally, searches for more exotic QCD objects, such as deuterons, glueballs, instantons and pentaquarks are briefly reviewed. Inclusive energy flow measurements have also been made at HERA, most recently and precisely in (Adloff et al., 2000d), but are not discussed further here.

\section{A. Charged Particle Distributions}

Numerous measurements of charged particles have been made at HERA, investigating both the hard and soft aspects of QCD. Whilst measurements of jet production probe higher scales and cover a wider kinematic range, the high statistics and precise reconstruction when measuring individual particles allows some more detailed tests. The basic kinematic distributions of all charged particles combined have been measured in both DIS (Aaron et al., 2007; Abt et al., 1994a; Adloff et al., 1997e; Alexa et al., 2013b; Derrick et al., 1995b, 1996a) and photoproduction (Abt et al., 1994b; Adloff et al., 1999a; Breitweg et al., 1998a) and are in general described by Monte Carlo models incorporating leadingorder matrix elements and parton showers followed by hadronisation. Further measurements have been made in which specific particle species are tagged, e.g. $K_{S}^{0}$ mesons, or different distributions or properties are investigated, such as angular correlations. The body of work is too great to cover in this short section and the reader is referred to the large number of relevant papers on the H1 (H1, 1992-) and ZEUS (ZEUS, 1992-) paper lists. In this section a few results are discussed, focusing on comparisons with data from other reactions and the general conclusions which can be drawn as a result, as well as comparisons with NLO QCD predictions using fragmentation functions (the probability that a parton hadronises into a given hadron which carries a fraction $z$ of the parton's momentum).

Charged particle multiplicities have been measured in many experiments, at $p \bar{p}, p p, e p$ and $e^{+} e^{-}$colliders (Beringer et al., 2012), testing models of fragmentation and the universality of the process. In ep DIS scattering, there are several combinations of scales and frames that can be used (Aaron et al., 2007; Adloff et al., 1997c, 1998b; Aid et al., 1996a; Breitweg et al., 1999d; Chekanov et al., 2001a, 2008d; Derrick et al., 1995c). In the results reviewed here (Chekanov et al., 2008d) the final state was reconstructed in the Breit and hadronic centre-of-mass frames in which respectively twice the energy of the current region of the Breit frame, $2 \cdot E_{\mathrm{B}}^{\mathrm{cr}}$, and the $\gamma^{*} p$ centre-of-mass energy, $W$, were used as scales. The charged particle multiplicities in DIS for these different frames and their respective energy scales are shown in Fig. 33 in comparison with previous DIS experiments and results from $e^{+} e^{-}$experiments. In general charged particle spectra at HERA show the same trend as those from other experiments over about two orders of magnitude in the energy scale of the interaction. In particular, the data from the two $e p$ frames with their respective energy scales agree well with the results from $e^{+} e^{-}$experiments, in contrast to when the scale $Q$ is used in the Breit frame (Breitweg et al., 1999d). This results in a lower charged multiplicity at low values, $Q<10 \mathrm{GeV}$, than both the $e^{+} e^{-}$data and the DIS data when $2 \cdot E_{\mathrm{B}}^{\text {cr }}$ is used as the scale. The fixed-target DIS data agree with the HERA and $e^{+} e^{-}$data at low scales, but increasingly deviate above about $15 \mathrm{GeV}$.

A related quantity considered is the number of charged particles per event per unit scaled particle momentum, $x_{p}=2 P_{\text {Breit }} / Q$, where $P_{\text {Breit }}$ is the momentum of a hadron in the Breit frame. This quantity, measured in DIS (Aaron et al., 2007; Abramowicz et al., 2010e; Breitweg et al., 1997c), is shown in Fig. 34 over two orders of magnitude in $Q$ in bins of $x_{p}$, compared with $e^{+} e^{-}$ data. As $Q$ increases, the phase space for soft gluon radiation increases, leading to a rise of the number of soft particles with small $x_{p}$, which along with the decrease with $Q$ at high $x_{p}$ results in clear scaling violations. The comparison between HERA and $e^{+} e^{-}$data in Fig. 34 is good for all $Q$ and $x_{p}$ which, along with the comparison in Fig. 33 supports the concept of the universality of fragmentation. Positively and negatively charged particles have also been considered separately and the charge asymmetry measured (Aaron et al., 2009e) as a function of $Q$ and $x_{p}$. At large $x_{p}$, the observed charge asymmetry is found to increase with $Q$ and correspondingly with Bjorken- $x$, consistent with the expectation that this observable is related to the valence quark content of the proton.

The inclusive data are compared in Fig. 35] with NLO QCD calculations which use fragmentation functions obtained from fits to $e^{+} e^{-}$data. The predictions from the four different groups shown are relatively similar to each 


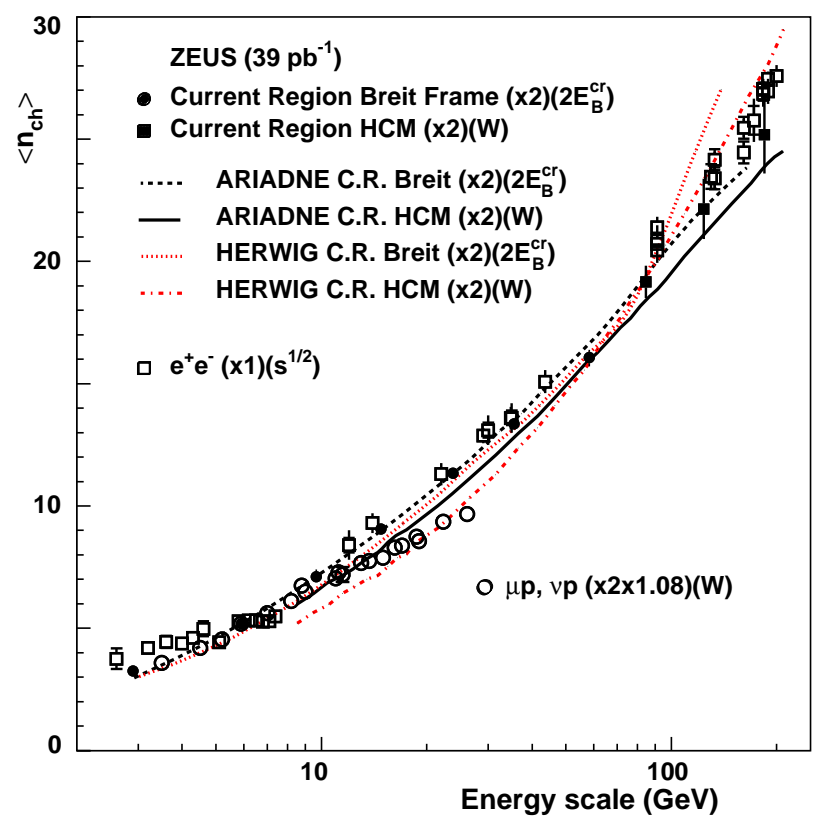

FIG. 33 Mean charged multiplicity, $\left\langle n_{\mathrm{ch}}\right\rangle$, as a function of the energy scale for ZEUS DIS measurements in the Breit and hadronic centre-of-mass frames, compared with results from $e^{+} e^{-}$and fixed-target DIS experiments. From (Chekanov et al., 2008d) and references therein for fixed-target and $e^{+} e^{-}$data.

other but do not describe the data well. The scaling violations are poorly described, with the theory having a shallower rise for low $x_{p}$ than the data. Also, the theory predicts too many particles at low $x_{p}$, whereas too few are predicted at high $x_{p}$. The disagreements between data and theory are, in general, in kinematic regions outside those measured in $e^{+} e^{-}$collisions, such as low $Q^{2}$ or high $x_{p}$. Hence the theory, which is derived from fits to the $e^{+} e^{-}$data, is extrapolated to regions in which it may not be applicable. Future fits should therefore use these HERA data to further constrain the fragmentation functions.

\section{B. Bose-Einstein Correlations}

In 1959, Goldhaber et al. (Goldhaber et al., 1959, 1960) observed that pairs of like-sign pions in $p \bar{p}$ collisions had a tendency to have smaller opening angles than pairs of unlike-sign pions. This is interpreted as being due to the symmetrisation of the wave-functions of pairs of identical bosons and is hence known as Bose-Einstein correlations (BEC). The effect has since been studied in various hadron-hadron collisions, $e^{+} e^{-}$annihilation and lepton-nucleon scattering (Alexander, 2003). The BEC in momentum space are related to the spatial dimensions of the production source, with measurements usually being characterised in terms of the effective source size, $r$.

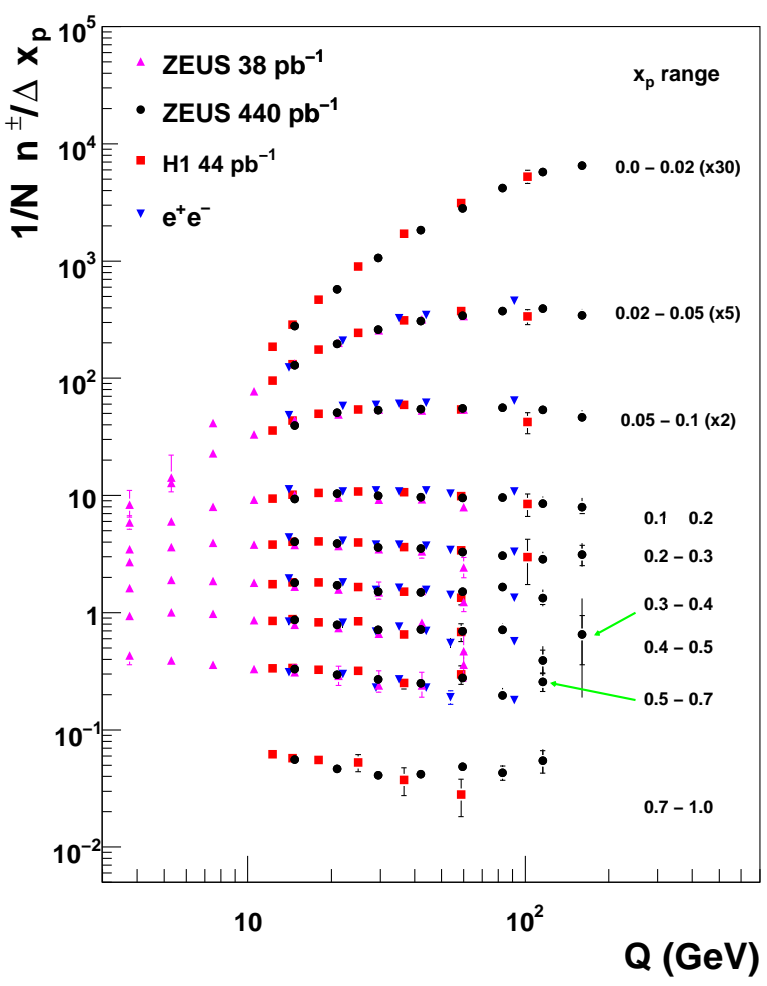

FIG. 34 The number of charged particles per event per unit $x_{p}$ as a function of $Q$ in bins of $x_{p}$. Data are shown for H1 and ZEUS and are compared with those from $e^{+} e^{-}$experiments (Abreu et al., 1993; Braunschweig et al., 1990; Li et al., 1990; Petersen et al., 1988). From (Abramowicz et al., 2010e).
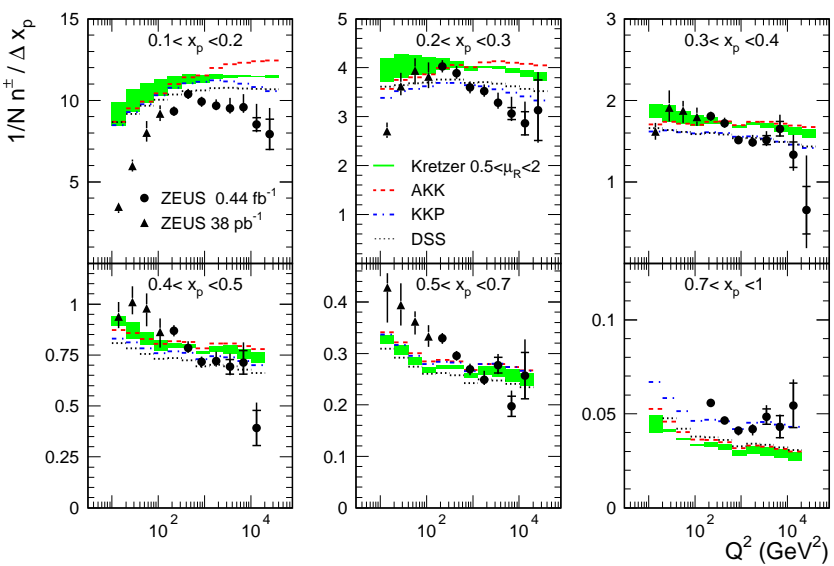

FIG. 35 The number of charged particles per event per unit $x_{p}$ as a function of $Q$ in bins of $x_{p}$. The shaded band represents an NLO QCD calculation by Kretzer (Kretzer, 2000) with its renormalisation uncertainty. Additional calculations are shown: Kniehl, Kramer and Pötter (KKP) (Kniehl et al., 2000); Albino, Kniehl and Kramer (AKK) (Albino et al., 2005, 2008); and De Florian, Sassot and Stratmann (DSS) (de Florian et al., 2007a b) . From (Abramowicz et al., 2010e). 
Whilst measurements of $r$ clearly reflect the size of the interacting particles in heavy ion collisions (Aamodt et al., 2011), the situation from proton-proton collisions is less clear, with dependences on the event multiplicity and transverse momentum of the bosons observed in high energy experiments (Aamodt et al., 2010; Alexopoulos et al., 1993; Khachatryan et al., 2011). Conclusions from BEC studies are to some extent obscured by the fact that the extracted values of $r$ are often strongly dependent on the choice of control sample relative to which the signal is measured. Values obtained using unlike-sign control samples are up to a factor of two larger than those obtained relative to event-mixed control samples.

In $e p$ scattering, the source size might be expected to reflect the size of the proton in low energy-scale processes and to evolve to smaller sizes more similar to those from $e^{+} e^{-}$data as the energy scale increases and the electron scatters from a single parton rather than from the proton as a whole. It is therefore interesting to study whether there is any dependence of the effective source size on the kinematic variables, in particular $Q^{2}$, since this reflects the effective transverse size of the exchanged virtual photon. The HERA case is therefore ideally suited to investigating whether $r$ depends on the primary interaction or whether it is determined solely by the fragmentation stage of the process.

Measurements of BEC at HERA have been performed for various different samples and kinematic regions in DIS, spanning $0.1<Q^{2}<8000 \mathrm{GeV}^{2}$. H1 and ZEUS have both measured the effect using an inclusive sample of hadrons (Adloff et al., 1997a; Chekanov et al., 2004b). H1 additionally studied a diffractive DIS event sample (Adloff et al., 1997a), in order to investigate whether a difference between the production mechanisms of diffractive and non-diffractive processes could be established. Motivated by a reported dependence of $r$ on the mass of the interfering bosons in $e^{+} e^{-}$annihilation $\left(r_{\pi \pi}>r_{K K}>r_{\Lambda \Lambda}\right.$ (Alexander et al., 1999) $)$, ZEUS also measured the effect for samples of charged and neutral kaons (Chekanov et al., 2007a).

Selected effective source size measurements from DIS and $e^{+} e^{-}$collisions are collected in Table I. No dependence of the source size on the kinematic variables, including $Q^{2}$, was found at HERA. There is also no significant variation between the results obtained in diffractive and inclusive DIS. The results for charged and neutral kaons are consistent with one another and also with those from inclusive charged particle production. Furthermore, the results from HERA are consistent with lower-energy DIS experiments (Arneodo et al., 1986; Korotkov et al., 1993). HERA and the other DIS data are also compatible with measurements from $e^{+} e^{-}$ annihilation (Alexander et al., 1999; Althoff et al., 1986; Choi et al., 1995; Juricic et al., 1989). From these observations, it can be concluded that, within the DIS regime, Bose-Einstein interference in $e p$ scattering does not depend significantly on the details of the hard process. The similarity with data from $e^{+} e^{-}$annihilation further indicates that the presence of an incoming hadron is not important if a short distance hard interaction takes place. The effective source size is thus driven primarily by the fragmentation process in DIS.

\begin{tabular}{|c|c|c|}
\hline Process & Experiment & $r(\mathrm{fm})$ \\
\hline \hline \multirow{3}{*}{$e^{+} e^{-}$} & AMY & $0.73 \pm 0.05 \pm 0.20$ \\
\cline { 2 - 3 } & TASSO & $0.82 \pm 0.06 \pm 0.04$ \\
\cline { 2 - 3 } & MARK II & $0.75 \pm 0.03 \pm 0.04$ \\
\cline { 2 - 3 } & LEP & $0.78 \pm 0.01 \pm 0.16$ \\
\hline \hline \multirow{3}{*}{$\begin{array}{c}\text { Previous } \\
\text { DIS }\end{array}$} & EMC & $0.84 \pm 0.03$ \\
\cline { 2 - 3 } HERA & BBCNC & $0.80 \pm 0.04$ \\
\hline \hline \multirow{4}{*}{} & H1 & $0.68 \pm 0.04_{-0.05}^{+0.02}$ \\
\cline { 2 - 3 } & ZEUS & $0.666 \pm 0.009_{-0.036}^{+0.022}$ \\
\cline { 2 - 3 } & ZEUS $\left(K^{ \pm} K^{ \pm}\right)$ & $0.57 \pm 0.09_{-0.08}^{+0.05}$ \\
\cline { 2 - 3 } & ZEUS $\left(K_{S}^{0} K_{S}^{0}\right)$ & $0.63 \pm 0.09_{-0.08}^{+0.11}$ \\
\hline
\end{tabular}

TABLE I Table of source sizes, $r$, extracted from hadron production in $e^{+} e^{-}$annihilation and in lepton-nucleon scattering. The uncertainties given for EMC and BBCNC are statistical only; the systematic uncertainties are expected to be of a similar size. All other results have the statistical and systematic uncertainties shown separately. The control samples used are the unlike-sign samples except for the LEP average and ZEUS kaon measurements. The LEP value is the average of using the unlike-sign and event-mixing samples as the control with the second error reflecting half the difference between the two and the value when using the unlike-sign sample is the higher of the two $r$ values. For the kaon pairs, an unlike-sign sample is not usable due to the strong signal from $\phi \rightarrow K^{+} K^{-}$.

\section{Charm Fragmentation}

Predictions of observable heavy quark cross sections rely on a knowledge of fragmentation parameters. This is particularly true when measuring the production rate of a given e.g. charm meson where both the fraction of charm quarks hadronising to the given meson and the charm fragmentation function are required inputs in a QCD calculation. Due to the large charm cross section at HERA, precise measurements of charm fragmentation parameters have been performed. The parameters can be compared with results from $e^{+} e^{-}$data, thereby testing the universality of the charm fragmentation process. As well as the ground state charm mesons, excited charm mesons (e.g. $D_{1}$ and $D_{2}^{*}$ ) have also been reconstructed and fragmentation parameters extracted. These are not discussed further here and the reader is referred to the relevant publications (Abramowicz et al., 2013d; Chekanov et al., 2009i).

The probability that a charm quark hadronises into a given hadron, the fragmentation fraction, has been de- 
termined at HERA for all ground state charm hadrons, both in DIS (Abramowicz et al., 2010d; Aktas et al., 2005a; Chekanov et al., 2007k) and in photoproduction (Abramowicz et al., 2013b; Chekanov et al., 2005c). The results are shown in Fig. 36 along with a combined result from $e^{+} e^{-}$annihilation (Gladilin, 1999; Lohrmann, 2011). The results for a given hadron are consistent between the three processes and, particularly in the photoproduction regime, the HERA results are of competitive precision to the results from $e^{+} e^{-}$data.

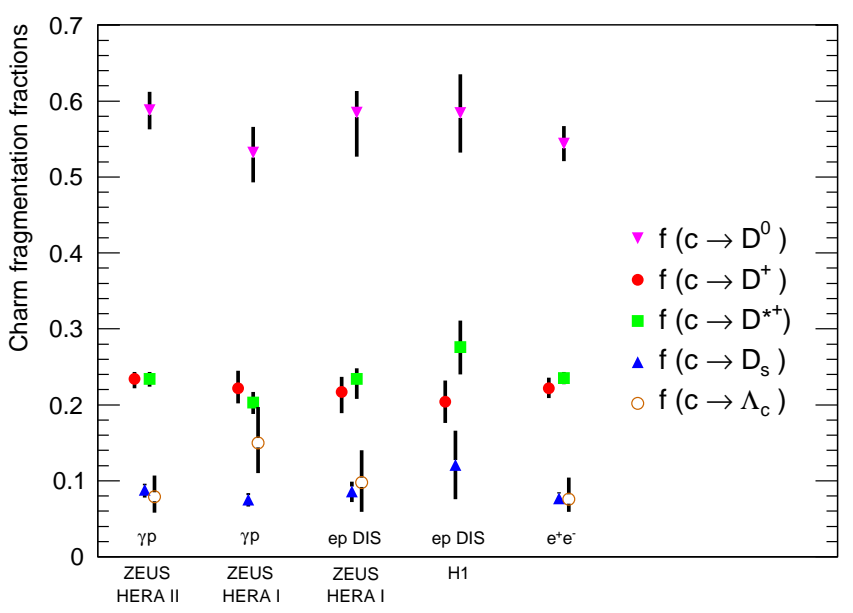

FIG. 36 Measurements of fragmentation fractions for various charm hadrons in DIS and photoproduction at HERA and also in $e^{+} e^{-}$annihilation at LEP. From Abramowicz et al., 2013b)

As the values of the fragmentation fractions are consistent with being independent of the process, all measurements have been combined (Lohrmann, 2011). The results of this analysis represent our best knowledge of these values and should be used in future calculations of charm production.

The so-called fragmentation function of a given hadron is the distribution in the fractional transfer $z$ of a quark's energy to the hadron. This has been measured for the $D^{*}$ meson in DIS by H1 (Aaron et al., 2009f) and in photoproduction by ZEUS (Chekanov et al., 2009g). Both sets of results showed a strong sensitivity to models of fragmentation with parameters in a given model varied and best-fit values extracted. The measurement from ZEUS tagged $D^{*}$ mesons associated to a jet; the relatively high scale of the process, $\sqrt{\hat{s}}$, given by $2 \cdot\left\langle E_{T}^{\text {jet }}\right\rangle=$ $23.6 \mathrm{GeV}$, allowed a measurement of the fragmentation function which was relatively unbiased by the kinematic cuts (Chekanov et al.,2009g). The ZEUS data are shown in Fig. 37 compared to results from $e^{+} e^{-}$collisions. The scales of the process for Belle and CLEO are similar, about $10.5 \mathrm{GeV}$, whereas for ALEPH the scale is 91.2 GeV. It can be seen that the ZEUS data, which are intermediate in scale, lie between the Belle/CLEO and
ALEPH results, which is qualitatively consistent with expectations from scaling violations in QCD in which $\langle z\rangle$ decreases with increasing energy (Cacciari et al., 2006). Similarly, the $z$ distributions from both collaborations were used to extract the parameter $\epsilon$ in the Peterson fragmentation function (Peterson et al., 1983) as implemented in the Pүтнia Monte Carlo model and the outcomes were found to agree with the results when fitting the $e^{+} e^{-}$data. These results are consistent with the hypothesis of fragmentation universality between $e p$ and $e^{+} e^{-}$processes.

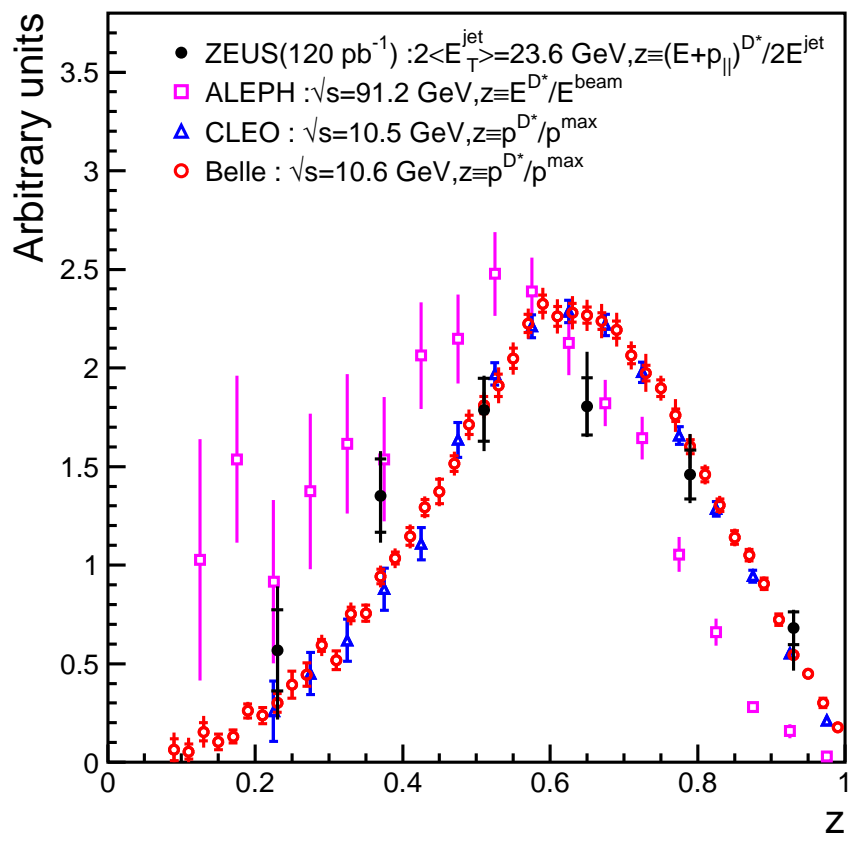

FIG. $37 D^{*}$ fragmentation function $f(z)$ for ZEUS data compared to measurements in $e^{+} e^{-}$collisions. To compare the shapes, the data sets were normalised to $1 /$ (bin width) for $z>0.3$. From (Chekanov et al., 2009g).

Both collaborations fit NLO QCD predictions to the data using the Kartvelishvili et al. model (Kartvelishvili et al., 1978) for fragmentation,

$$
f(z) \propto z^{\alpha}(1-z)
$$

where $\alpha$ is a free parameter. The result from ZEUS of $\alpha=2.67_{-0.31}^{+0.25}$ can be compared with that from H1 for lower scales, given in Fig. 38, A higher value of $\alpha$ implies a high $\langle z\rangle$ and so the observation of $\alpha$ increasing with decreasing scale, $\sqrt{\hat{s}}$, is also consistent with the expectations from scaling violations in QCD. However, it should be noted that the quality of the fit for the lowest scale, in Fig. [38(b), is poor indicating an incomplete description of the full phase space down to the production threshold. Future fits of fragmentation functions should use these HERA data as part of their input and the uncertainties in the determination of the fragmentation parameter 
should ideally be propagated as a systematic uncertainty in NLO QCD calculations of a given charm cross section.
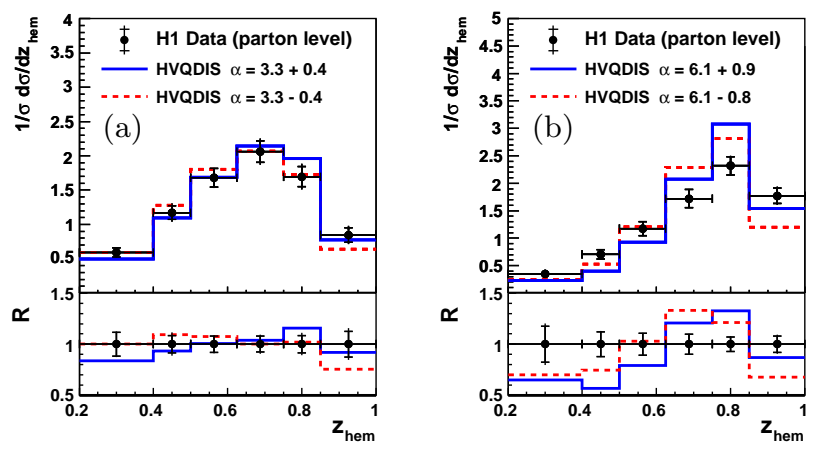

FIG. 38 Normalised $D^{*}$ cross sections as a function of an estimator, $z_{\text {hem }}$, of the fragmentation function, $z$, showing the best fits of an NLO QCD prediction using the Kartvelishvili fragmentation function with the uncertainty range on the $\alpha$ parameter shown. The scales of the processes are (a) $8.4<$ $\sqrt{\hat{s}}<18 \mathrm{GeV}$ and (b) $\sqrt{\hat{s}}<8.4 \mathrm{GeV}$. From Aaron et al. 2009f).

\section{Inelastic $J / \psi$ Production}

Even though the discovery of the $J / \psi$ meson, and hence the charm quark, was made in 1974, its hadroproduction mechanism is still uncertain (for a review, see (Brambilla et al., 2011)). At HERA, $J / \psi$ production is dominated by boson-gluon fusion in which a photon emitted from the incoming electron interacts with a gluon from the proton to produce a $c \bar{c}$ pair which subsequently forms a $J / \psi$ meson. In the coloursinglet (CS) model (Baier and Ruckl, 1981, 1982, 1983; Berger and Jones, 1981; Chang, 1980), the $c \bar{c}$ pair produced has the same quantum numbers as the physical $J / \psi$ bound state, achieved by radiating a hard gluon in the perturbative process. In the colour-octet $(\mathrm{CO})$ model (Bodwin et al., 1995; Caswell and Lepage, 1986; Thacker and Lepage, 1991), the $c \bar{c}$ pair emerges from the hard process with quantum numbers different from those of the $J / \psi$ and evolves into the physical $J / \psi$ state by emitting one or more soft gluons. The probability for $\mathrm{CO}$ processes occurring is governed by long-distance matrix elements that can be obtained from fits to experiment. Predictions within the CS framework have also been made using the $k_{T}$ factorisation approach (Baranov, 1998, 2002; Lipatov and Zotov, 2003) in which the effects of non-zero incoming parton transverse momentum are taken into account. These three approaches have been compared extensively to HERA measurements.

Both $\mathrm{H} 1$ and ZEUS have measured inelastic $\mathrm{J} / \psi$ production in DIS (Aaron et al., 2010d; Adloff et al., 2002d; Chekanov et al., 2005d) and in photoproduction (Aaron et al., 2010d; Abramowicz et al., 2013c; Adloff et al., 2002d; Ahmed et al., 1994b; Aid et al., 1996b; Breitweg et al., 1997a; Chekanov et al., 2003e, $2009 \mathrm{f})$. Inelastic $J / \psi$ production has also been measured extensively at the Tevatron and the resulting data have been used to constrain the CS and CO contributions, usually by first applying a CS calculation and then adding a CO contribution, fitted to best describe the data. By combining the $\mathrm{CS}$ and fitted $\mathrm{CO}$ contributions in this manner, the theory is able to describe the $J / \psi$ cross section in $p \bar{p}$ collisions (Cho and Leibovich, 1996a b). However neither this, nor the $k_{T}$ factorisation approach are able to describe the polarisation measurements for $\mathrm{J} / \psi$ and $\psi(2 S)$ mesons (see (Abulencia et al., 2007) and references therein). The CO contribution fitted to the Tevatron data is expected to be applicable to ep collisions and is used to predict $\mathrm{H} 1$ and ZEUS data. A measurement of $J / \psi$ photoproduction is shown in Fig. 39 in comparison to LO and NLO QCD predictions (Artoisenet et al., 2009; Butenschoen and Kniehl, 2010, 2011a,b; Kramer, 1996) from the CS model and the CS and CO models combined. The predictions from the CS model lie below the data although the shapes of the $P_{T}^{2}$ and inelasticity, $z$ (not shown), distributions are well reproduced. As the uncertainties on the theoretical predictions are large, the next order in QCD may be required to describe the data. Inclusion of a $\mathrm{CO}$ contribution gives a higher cross section and describes the data well, although again with large uncertainties. Predictions based on $k_{T}$ factorisation in the CS model (using the CASCADE Monte Carlo programme (Jung, 2002; Jung and Salam, 2001), not shown) also gives a good description of the data.

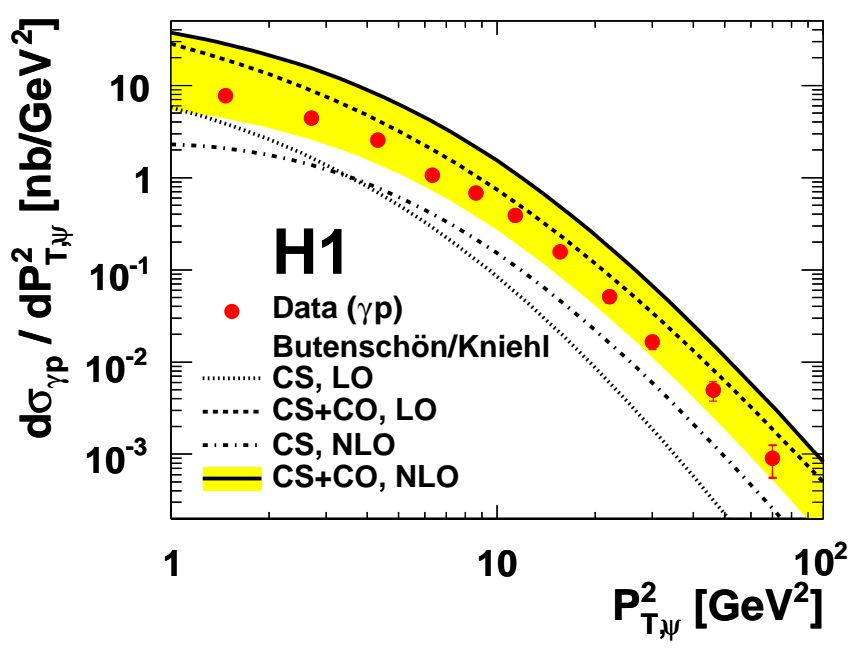

FIG. 39 Photoproduction cross section differential in $P_{T}^{2}$ of the $J / \psi$ meson for $\mathrm{H} 1$ data, compared with $\mathrm{LO}$ and NLO QCD predictions from the CS model and the CS and $\mathrm{CO}$ models combined. The uncertainty on the theory (yellow band) arises from the difference between results using an LO or a higher-order colour octet contribution (Butenschoen and Kniehl, 2010). From (Aaron et al., 2010d).

Measurements of the polarisation of the $J / \psi$ mesons 
provided strong distinguishing power for the Tevatron results and comparable analyses were performed at HERA. An example is shown in Fig. 40, where the helicity parameter is plotted versus the inelasticity for the full ZEUS data sample. The theoretical and experimental uncertainties are large. None of the predictions gives a good description over the full phase space and unfortunately discrimination between different models is not possible from the HERA data alone.

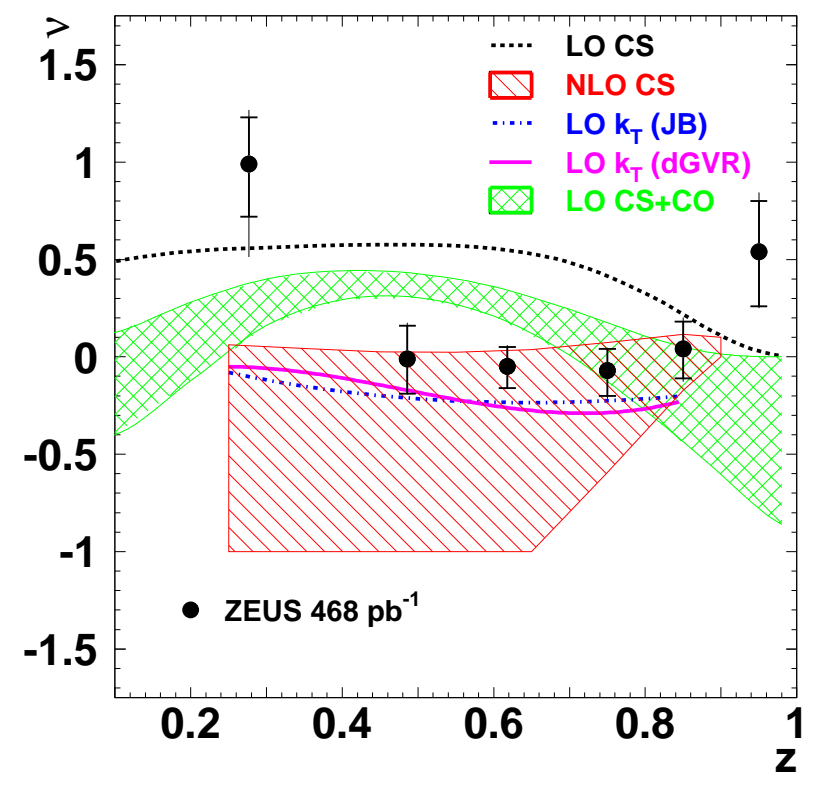

FIG. 40 Helicty parameter, $\nu$, for the decay azimuthal angle in the frame where the polarisation axis in the $J / \psi$ meson rest frame is defined by the flight direction of the $J / \psi$ meson in the $\gamma p$ rest frame. The value can vary between -1 and +1 and is shown here for different inelasticity values. From (Chekanov et al., 2009f).

Unfortunately, the production mechanism for $J / \psi$ mesons remains largely unresolved. Although HERA has produced precise measurements of differential cross sections in DIS and photoproduction and measurements of the polarisation, progress is limited by the large theoretical uncertainties. Further measurements from the Tevatron or LHC (Aad et al., 2011a; Aaii et al., 2011; Abelev et al., 2012a,b; Chatrchvan et al., 2012a) extending to higher $P_{T}$ along with global analyses (see e.g. (Butenschoen and Kniehl, 2012)) including the HERA data presented here may lead to a deeper insight.

\section{E. Exotic and Unusual QCD}

Many of the searches for new physics at HERA have focused on the hadronic final state. The situation is perhaps well summarised by generic searches, which identify deviations between data and predictions for arbitrary combinations of high transverse momentum objects (leptons, photons, jets and missing energy) (Aaron et al., 2009a). Both here and in dedicated studies (Adloff et al., 1998c), H1 data consistently exhibited an excess of events with a single isolated lepton, a high transverse momentum jet and large missing energy, corresponding to the topology expected from a $W$ boson produced at high $p_{T}$, recoiling against a quark or gluon. However, no comparable signal was observed by ZEUS and excitement about this channel diminished when the full H1 data became available. The final situation from HERA is summarised in a combined H1 and ZEUS result (Aaron et al., 2010c). Ultimately HERA data across a wide range of signatures and channels are remarkably consistent with the Standard Model.

Within the Standard Model, a variety of previously unobserved quark and gluon bound states and other novel strong interaction phenomena are predicted. Due to its rich, complex and non-Abelian nature, the status of these states and phenomena within QCD is not always obvious. As discussed in the following sections, in many such cases observing new effects for the first time would constitute a major discovery in its own right.

\section{Pentaquark States}

The possibility to have particles consisting of five quarks and antiquarks, i.e. pentaquarks, was proposed as early as the 1970s Jaffe, 1977; Lipkin, 1987; Strottman, 1979) and is predicted within QCD (Diakonov et al., 1997). In the early part of the 2000s, many collaborations searched for pentaquark states, with several seemingly clear signals, but also contradictory results. The world-wide status is summarised in dedicated reviews of the subject, see for example (Danilov and Mizuk, 2008; Dzierba et al., 2005; Hicks, 2005a, b, 2012). Here we concentrate on the $\mathrm{H} 1$ and ZEUS contributions.

Most pentaquark searches have focussed on the lightest irreducible quark combination, termed $\Theta^{+}$, which has a quark content $u u d d \bar{s}$ and a mass expected to be around $1530 \mathrm{MeV}$. The ZEUS Collaboration searched (Chekanov et al., 2004c) for the $\Theta^{+}$in the $K_{S}^{0} p$ decay channel and reported a signal of around $4-4.5 \sigma$ significance with a mass of $1521 \pm 1.5 \mathrm{MeV}$ in DIS events. Superficially this was consistent with other 'observations' of the $\Theta^{+}$pentaquark. However the comparatively low mass (Hicks, 2005b) pointed to problems. H1 subsequently performed the same analysis Aktas et al., 2006i) and saw no signal, which, along with other null results (Danilov and Mizuk, 2008; Dzierba et al., 2005; Hicks, 2005a, b, 2012) and the fact that ZEUS only saw this at high $Q^{2}$ (above $20 \mathrm{GeV}^{2}$, but not above $1 \mathrm{GeV}^{2}$ ) indicates that the ZEUS result had some flaw or must have been a statistical fluctuation. ZEUS have yet to confirm or refute the result even though a data sample several times larger is available.

Both collaborations searched in the $\Xi \pi$ channel (Aktas et al., 2007e; Chekanov et al., 2005f) for the 
pentaquark resonance reported by the NA49 Collaboration (Alt et al., 2004). The search was performed for pentaquarks decaying to the doubly charged $\Xi^{-} \pi^{-}$and the neutral $\Xi^{-} \pi^{+}$final states. The HERA collaborations observed a clean resonance for the known baryon $\Xi(1530)^{0}$ decaying to $\Xi^{-} \pi^{+}$, but observed no other higher-mass resonances and so set production limits in the mass range, $1600-2350 \mathrm{MeV}$. These null results were in keeping with the many others for this channel (again see pentaquark review articles (Danilov and Mizuk, 2008; Dzierba et al., 2005; Hicks, 2005a, b, 2012) and references therein).

Assuming the existence of a light pentaquark such as the $\Theta^{+}$, then a heavier analogue with a charm quark might also be expected to exist. The H1 Collaboration searched for a $D^{*} p$ resonance and found a clear signal (Aktas et al., 2004a) at about $3.1 \mathrm{GeV}$, consistent

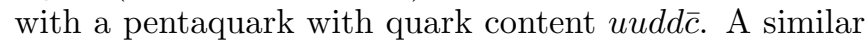
search was performed by ZEUS with a larger statistical sample (Chekanov et al., 2004h), but no signal was observed. Given the cleanliness of the H1 signal and its significance of at least $5-6 \sigma$, the situation was puzzling. Further null results were published by other high energy physics experiments (Danilov and Mizuk, 2008) and a higher-statistics analysis presented by H1 (Krueger, 2009). Although unpublished, the newer H1 measurement shows no resonance structure around $3.1 \mathrm{GeV}$, using a data sample four times larger than previously, suggesting that the original results arose from a statistical fluctuation.

In summary, after initial excitement and 'discoveries' of new particles, the results from HERA have gone the way of the overall status for pentaquark searches in the many experiments world-wide (Amsler et al., 2008; Hicks, 2012), with no convincing evidence remaining.

\section{Instanton Searches}

In both electroweak and QCD interactions, the ground state has a rich topological structure, associated with non-perturbative fluctuations of the gauge fields, called instantons (Belavin et al., 1975; 't Hooft, 1976a, b). In electroweak interactions, instantons are not expected to be observable at current colliders. In QCD, the effects of instantons are expected to be manifest at lower energies (Balitsky and Braun, 1993; Moch et al., 1997; Ringwald and Schrempp, 1994, 1999), although they have yet to be seen. The rate of instanton production in DIS at HERA was expected to be sizeable (Gibbs et al., 1995; Moch et al., 1997; Ringwald and Schrempp, 1994, 1998, 1999, 2000, 2001) and so presented the HERA experiments with an opportunity to discover the effect. The major challenge for $\mathrm{H} 1$ and ZEUS (Adloff et al., 2002g; Chekanov et al., 2004i) was that the instanton cross section was nonetheless small in comparison with the inclusive, which was hard to suppress sufficiently.

The expected characteristics of instanton events are large transverse energy, high-multiplicity and the pro-
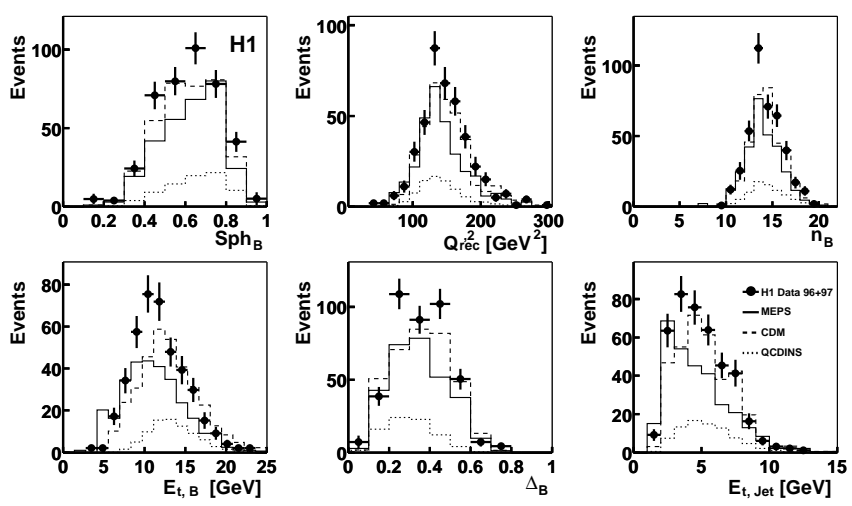

FIG. 41 Raw distributions in sphericity, $Q^{2}$, charged particle multiplicity, total transverse energy, isotropy and transverse jet energy, compared with predictions for DIS events and for instanton-induced events (QCDINS), after applying a cut on a multivariate discriminant designed to enhance instanton-like signatures. From (Adloff et al., 2002g).

duction of different quark flavours democratically and isotropically in their centre-of-mass frame (a 'fireball' configuration). A search based on these criteria is presented in Fig. 41, which compares the predicted normalisations and the shapes for particular variables between data, the QCDINS instanton model (Ringwald and Schrempp, 2000) and inclusive DIS models after applying a cut based on a multivariate discriminant. The application of cuts such as this suppresses the inclusive DIS background by factors of typically 1000 . Although events exhibiting instanton-like characteristics are observed, their isolation is hampered by the uncertainty on the remaining background, mainly due to the sometimes poor modelling of the hadronic final state by the inclusive DIS Monte Carlo simulations. It has therefore not been possible to isolate regions in which a clear signature for instanton production is expected. However, the analysis allows upper limits to be placed on instanton production cross sections which are within a factor of $3-5$ of the predicted values, depending on the kinematic region. Tuning and improving the simulation of the hadronic final state using the measurements contained in this article may allow a signal for instanton production to be extracted in other experiments.

\section{Search for Glueball States}

The existence of glueballs (bound states of two or more gluons) is predicted by QCD. The lightest state is expected to have quantum numbers $J^{P C}=0^{++}$and a mass in the range $1550-1750 \mathrm{MeV}$ (Yao et al., 2006). The state $f_{0}(1710)$ is frequently considered to be a glueball or tetraquark candidate (Albaladejo and Oller, 2008; Klempt and Zaitsev, 2007), since it does not fit into existing multiplet structures, but its constituent parton content is not established. As the $K_{S}^{0} K_{S}^{0}$ system is ex- 
pected to couple to glueballs, resonances in its invariantmass spectrum have been searched for.

In an initial study from ZEUS (Chekanov et al., 2004f) of the $K_{S}^{0} K_{S}^{0}$ system in DIS, indications of two states, $f_{2}^{\prime}(1525)$ and $f_{0}(1710)$, were seen with statistical significances of about three standard deviations. A subsequent measurement (Chekanov et al., 2008e) used all events and all data $\left(0.5 \mathrm{fb}^{-1}\right)$, dominated by photoproduction; given the gluon-rich environment, it is expected that photoproduction should be more sensitive than DIS to the production of glueballs. By reconstructing a secondary vertex and removing contamination from photon conversions and $\Lambda$ baryons, a clean sample of $K_{S}^{0}$ candidates were reconstructed via their decay to $\pi^{+} \pi^{-}$(Chekanov et al., 2008e). Using over $10^{6} K_{S}^{0}$ mesons, pairs were combined to give the invariant-mass spectrum shown in Fig. 42. Three peaks around 1300, 1500 and $1700 \mathrm{MeV}$ are observed with no heavier state seen.

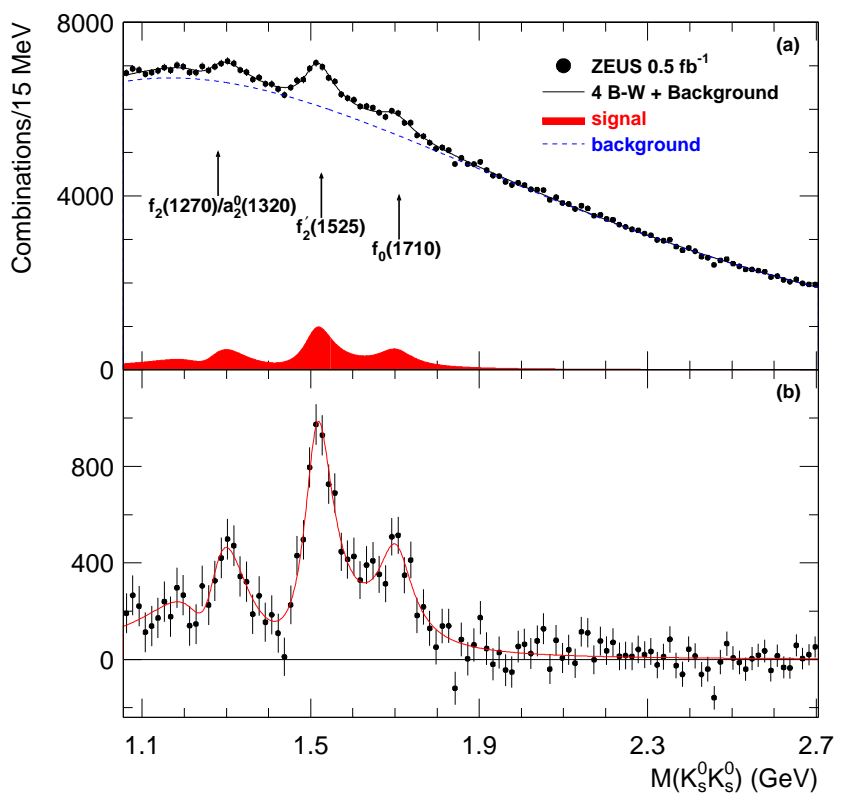

FIG. 42 (a) The measured $K_{S}^{0} K_{S}^{0}$ invariant-mass spectrum compared with a fit including signal and background contributions. (b) The background-subtracted $K_{S}^{0} K_{S}^{0}$ invariantmass spectrum with the fit for the signals also shown. From (Chekanov et al., 2008e).

The states were fitted accounting for the interference pattern predicted by $\mathrm{SU}(3)$ symmetry arguments (Faiman et al., 1975). The first peak at about $1300 \mathrm{MeV}$ is consistent with the combination $f_{2}(1270) / a_{2}^{0}(1320)$. The second and third peaks are consistent with the states $f_{2}^{\prime}(1525)$ and $f_{0}(1710)$; their masses and widths are consistent with values from the PDG (Yao et al., 2006) and are of similar precision. The fit yields $4058 \pm 820$ events for the $f_{0}(1710)$ resonance, constituting a statistical significance of five standard deviations. The $f_{0}(1710)$ has a mass consistent with a
$J^{P C}=0^{++}$glueball candidate, although if it is the same state as is seen in $\gamma \gamma \rightarrow K_{S}^{0} K_{S}^{0}$ (Acciarri et al., 2001; Althoff et al., 1983), it is unlikely to be a pure glueball state.

\section{Deuteron and Anti-Deuteron Production}

The production of light stable nuclei, such as deuterons $(d)$, in high-energy collisions is poorly understood. Anti-deuterons $(\bar{d})$ were first observed in 1965 (Dorfan et al., 1965; Massam et al., 1965) and subsequently in $e^{+} e^{-}$(Akers et al., 1995; Albrecht et al., 1985, 1990; Asner et al., 2007; Schael et al., 2006), proton-nucleus ( $p A)$ (Antipov et al., 1971; Binon et al., 1969; Cronin et al., 1975), protonproton ( $p p)$ (Abramov et al., 1987; Alper et al., 1973; Henning et al., 1978) and nucleus-nucleus (Adler et al., 2001, 2005; Ahle et al., 1998; Anticic et al., 2012; Aoki et al., 1992; Appelquist et al., 1996; Arsene et al., 2011; Bearden et al., 2002, 1999) collisions. The coalescence model (Butler and Pearson, 1963) was developed to describe the production of deuterons and anti-deuterons in heavy-ion collisions. This approach has also been used to describe $d$ or $\bar{d}$ production in $p p$ and $e^{+} e^{-}$interactions. The invariant differential cross section for deuteron production can be parametrised as

$$
\frac{E_{d}}{\sigma_{\text {tot }}} \frac{\mathrm{d}^{3} \sigma_{d}}{\mathrm{~d} p_{d}^{3}}=B_{2}\left(\frac{E_{p}}{\sigma_{\text {tot }}} \frac{\mathrm{d}^{3} \sigma_{p}}{\mathrm{~d} p_{p}^{3}}\right)^{2}
$$

where $E_{d(p)}$ and $\sigma_{d(p)}$ are the energy and the production cross section of the $d(p), p_{d}\left(p_{p}\right)$ is the momentum of the $d(p)$ and $\sigma_{\text {tot }}$ is the total cross section. The coalescence parameter, $B_{2}$, is inversely proportional to the volume of the fragmentation region emitting the particle. If $B_{2}$ is the same for particles and anti-particles, then the production ratio for $\bar{d} / d$ is expected to be equal to the square of that for $\bar{p} / p$.

The production of anti-deuterons was first measured at HERA by $\mathrm{H} 1$ in photoproduction (Aktas et al., 2004d); ZEUS then measured both deuteron and anti-deuteron production in DIS (Chekanov et al., 2007i). Both collaborations exploited the rate of ionisation energy loss, $\mathrm{d} E / \mathrm{d} x$, measured in the inner tracking chambers and the distance of closest approach of the track to the event vertex. Clear signals of $d$ and $\bar{d}$ were seen along with small signals for tritons; no heavier nuclei or anti-triton candidates were observed. After measurement of the invariant cross sections, the ratios of the different production rates were measured in order to compare with other colliders and with the coalescence model.

The ratio of production rates is shown in Fig. 43. The production rate of deuterons is three orders of magnitude smaller than the rate for protons. The ratios $d / p$ and $\bar{d} / \bar{p}$ and also the results in DIS and photoproduction in Fig. 43(a) are consistent with one another and 

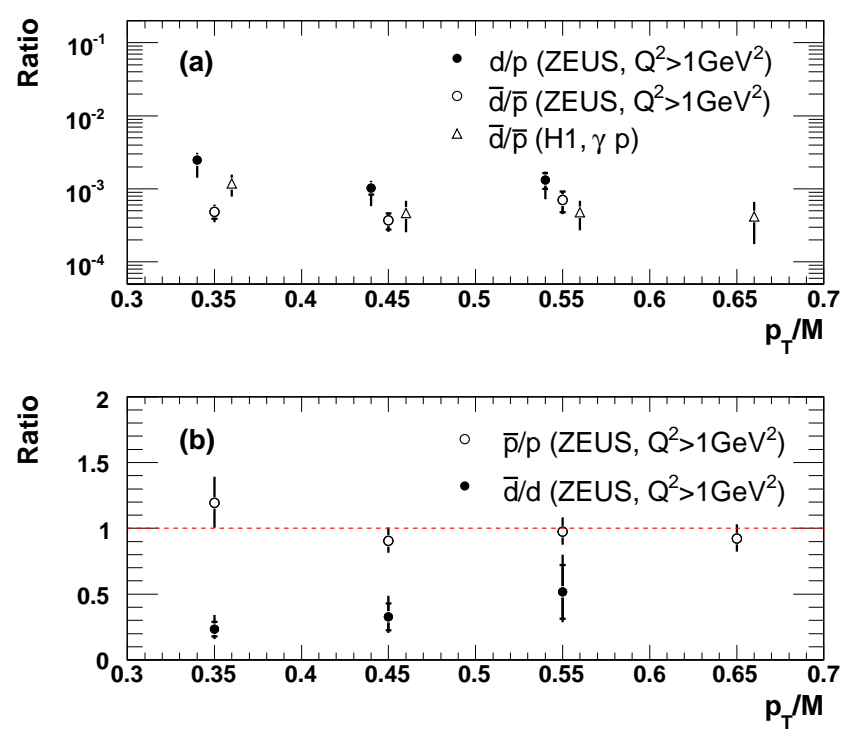

FIG. 43 (a) $d / p$ and $\bar{d} / \bar{p}$ production rates in DIS (ZEUS) and in photoproduction (H1) as a function of $p_{T} / M$. (b) $\bar{d} / d$ and $\bar{p} / p$ production rates in DIS as a function of $p_{T} / M$. From (Chekanov et al., 2007i).

with independence of the ratio, particle transverse momentum to mass, $p_{T} / M$. These ratios are also in agreement with those from $\Upsilon$ decays and $p p$ and heavy-ion data. Similarly when analysed within the context of the coalescence model, the value of $B_{2}$ extracted is consistent between $d$ and $\bar{d}$ and DIS and photoproduction. The results from HERA for $B_{2}$ agree with those from $p p$ data, but lie above the results from $e^{+} e^{-}$collisions at the $Z$ pole and from heavy-ion data. However, heavyion data at lower centre-of-mass energy tend towards the values from HERA and $p p$ collisions. In Fig. 43(b), the ratios $\bar{d} / d$ and $\bar{p} / p$ are shown versus $p_{T} / M$. The $\bar{p} / p$ ratio is consistent with unity as expected from hadronisation of quark and gluon jets. The ratio $\bar{d} / d$ is about $0.3 \pm 0.1$ and is inconsistent with the coalescence model, which predicts that this ratio should be equal to that for $(\bar{p} / p)^{2} \approx 1$, a relationship which is consistent with data from $p p$ (Abramov et al., 1987; Alper et al., 1973; Henning et al., 1978), pA (Cronin et al., 1975) and heavy-ion collisions (Adler et al., 2005). To summarise, the coalescence model can describe a number of features of $d$ and $\bar{d}$ production in high-energy collisions, but given the results here can not provide a complete picture.

\section{EXCLUSIVE AND SEMI-INCLUSIVE PROCESSES}

\section{A. Introduction}

Diffractive processes in proton-(anti)proton scattering, and to a lesser extent, other projectiles scattering diffractively on proton targets, have been the sub- ject of sustained and intense study since well before HERA. Early experimental results and their theoretical description are extensively covered in a number of review articles (Alberi and Goggi, 1981; Goulianos, 1983; Kaidalov, 1979; Zotov and Tsarev, 1988). The first results have also now started to appear from the LHC Aad et al., 2012b; Aaii et al., 2013; Abelev et al., 2013; Chatrchyan et al., 2013a). Although it was not widely expected prior to data taking, the study of quasielastic and diffractive processes has been one of the most successful areas of study at HERA, and certainly one of the most prolific in terms of publications. Complementary reviews can be found in (Ivanov et al., 2006; Wolf, 2010).

The kinematics of HERA, with a strong forward boost of the hadronic centre of mass relative to the lab frame for non-extreme $y$ values, were particularly favourable for the study of diffractive excitations of the real and virtual photon. Two separate cases are usually distinguished. In the (quasi-)elastic case, $\gamma^{(*)} p \rightarrow V p$, the photon coupling to the beam lepton either remains intact (Deeply Virtual Compton Scattering, DVCS) or converts to a vector meson $V$. In the diffractive dissociation case, $\gamma^{(*)} p \rightarrow X p$ the system $X$ produced at the proton vertex is a multiparticle state covering a continuum of invariant masses, $M_{X}$.

Most of the interest has centred around the case where the proton remains intact and is scattered through a small angle (implying a small absolute value of the squared four-momentum transfer at the proton vertex, $|t|$ (Figs. 45(a) and 52(a)). Measurements of processes in which the proton dissociates to large mass systems $Y$, i.e. $\gamma^{(*)} p \rightarrow X Y$ and $\gamma^{(*)} p \rightarrow V Y$ were more limited, but have been considered for example in the context of the decomposition of the total photoproduction cross section into diffractive and non-diffractive channels (Aid et al., 1995). The only detailed measurement of the $M_{X}$ dependence in proton dissociation processes Adloff et al., 1997b) revealed a clear difference compared with the elastic-proton case, which is presumably a consequence of the sub-leading exchanges discussed in Section V.D. but has yet to be interpreted in detail.

Prior to HERA, substantial data were already available on exclusive vector meson production in both DIS and photoproduction (Bauer et al., 1978; Binkley et al., 1982; Denby et al., 1984; Shambroom et al., 1982), with fixed target data continuing to emerge during and beyond the HERA era (Adams et al., 1997; Adolph et al., 2012; Airapetian et al., 2000, 2009; Alexakhin et al., 2007; Arneodo et al., 1994). DVCS has also recently been studied in detail in fixed target experiments at HERA (Airapetian et al., 2007, 2012a, b) and Jefferson Laboratory (Camacho et al., 2006; Gavalian et al., 2009; Girod et al., 2008). In contrast, the study of the diffractive dissociation of real and virtual photons is almost entirely the preserve of HERA, with only very limited fixed target data (Chapin et al., 1985).

Before the first precise measurements emerged from 
HERA, a QCD-based treatment of the exclusive production of vector mesons was already fairly well advanced (Brodsky et al., 1994; Frankfurt et al., 1996; Ryskin, 1993). Cross sections were expected to be $Q^{2}$-suppressed relative to inclusive DIS and to be related to the parton densities of the proton, the lowest order partonic exchange being a pair of gluons with compensating colour charges (Low, 1975; Nussinov, 1975). There was less consensus on the appropriate QCD treatment of single diffractive photon dissociation. On the one hand, 'hard' diffractive processes, similar to exclusive vector meson production were predicted, in which the colour singlet exchange couples fully into the hard interaction with the photon. Such an exchange may again be perturbatively calculable starting from a knowledge of the proton structure. In contrast, in 'soft' diffraction, the colour singlet exchange is a composite virtual object, similar to the pomeron of peripheral hadronic scattering, from which a single parton participates in the hard scattering, the remainder forming a low transverse momentum remnant system, cleanly separated in rapidity from the oppositelytravelling intact proton. This type of configuration (Donnachie and Landshoff, 1987; Ingelman and Schlein, 1985) produces a leading twist contribution with similar $Q^{2}$ dependence to the total cross section, limited only by kinematic constraints at large $Q^{2}$. In the DIS regime, it can be related to a concept of diffractive parton densities. A major issue in inclusive diffraction at HERA has been determining where each of these 'soft' and 'hard' processes are dominant and understanding the transition between the two.

The detailed dependence on centre-of-mass energy of elastic and, via the optical theorem, total hadronhadron cross sections has historically been remarkably well described in a large kinematic domain by Regge phenomenology (Regge, 1959, 1960). In this framework, interactions take place via the $t$-channel exchange of reggeons related to mesons (Chew et al., 1961) and of the leading vacuum singularity, the pomeron (IP) (Chew and Frautschi, 1961). The pomeron is the mediator of diffractive scattering. Although the pomeron is essentially a phenomenological object, associated mainly with soft processes, it can also be generated perturbatively. For example, connecting the two exchanged gluons in the lowest order partonic process by adding further gluon 'rungs' in a leading-logarithmic $1 / x$ approach leads to the so-called 'BFKL pomeron'.

At asymptotically large energies, pomeron exchange dominates the elastic channel, such that both elastic and total cross sections display a slow increase with centre-of-mass energy. Interactions in which one or both of the hadrons dissociates to higher mass states (Feinberg and Pomerančuk, 1956; Good and Walker, 1960) also occur naturally in this approach. Such processes are characterised by the presence of large regions of rapidity space in which no hadrons are produced and are dominated by diffractive exchange at large centre-of-mass energy $\sqrt{s}$ and small dissociation masses $M_{X}$. The inclusive dissociation mass distribution may be treated via Mueller's generalisation of the optical theorem (Mueller, 1970), such that an appropriate Regge description involves diagrams that contain three-reggeon couplings.

Pomeron language has been adopted to a large extent at HERA, as a matter of convenience. This should not be taken to imply the existence of a universal $t$-channel pomeron exchange, since the detailed properties of the exchange and its Regge trajectory clearly vary between processes and with kinematic variables at HERA, as discussed in detail below.

First evidence for diffractive dissociation processes involving sufficiently large scales for perturbative QCD to be applied came from diffractive dijet production in $p \bar{p}$ collisions at the SPS as observed by UA8 (Brandt et al., 1992). Later data from the Tevatron followed - see e.g. (Affolder et al., 2000). These observations lent experimental support to the idea that the pomeron might be considered as an object with its own structure, which might be probed in DIS (Donnachie and Landshoff, 1987; Ingelman and Schlein, 1985). First ideas on the structure of the pomeron were in place as early as the mid-1980s (Berger et al., 1987; Gribov et al., 1983).

Alternatively to Regge theory, an $s$-channel picture (Dederichs and Faessler, 1989; Miettinen and Pumplin, 1978) developed around the original Good \& Walker approach (Good and Walker, 1960) in which different projectile Foch states are absorbed differently on the target. These ideas have enjoyed a recent revival as part of the quest to describe diffraction at the LHC (Ryskin et al., 2011). The application of this $s$-channel approach to HERA data involves the elastic and quasi-elastic scattering of colour dipoles, corresponding to $q \bar{q}$ (or higher multiplicity) fluctuations of real and virtual photons on the proton (Mueller, 1990; Nikolaev and Zakharov, 1991). An attractive feature of this approach is that the possible presence of 'saturation' phenomena, whereby the low $x$ growth of parton densities is tamed as ultimately required to satisfy unitarity, are easily incorporated at the parametric level (Golec-Biernat and Wusthoff,,1998). Whilst such models were originally devised to describe the total $\gamma^{*} p$ cross section (see Fig. 44), ${ }^{2}$ vector meson production and other exclusive processes are easily incorporated with no further free parameters except those that describe the $t$ dependence (see e.g. (Kowalski et al., 2006; Kowalski and Teaney, 2003) for a modern treatment). Diffractive dissociation can also be included (Nikolaev and Zakharov, 1992), though has proved more difficult to describe, due to the need to incorporate higher terms, corresponding for example to a dipole formed from a $q \bar{q}$ pair and a gluon (Bartels et al.

\footnotetext{
2 The results from the inclusive cross section have been inconclusive to date, with strong evidence for saturation only at low scales $\ll 1 \mathrm{GeV}^{2}$, which preclude a partonic interpretation (Forshaw et al., 1999).
} 
1999a; Golec-Biernat and Wusthoff, 1999). The dipole approach to diffraction at HERA is discussed further in Sections V.B.5 and V.C.8

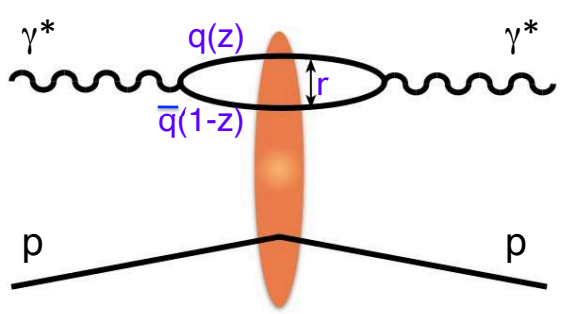

FIG. 44 Schematic illustration of the dipole model concept, in which a virtual photon fluctuates to a $q \bar{q}$ pair with relative momentum fractions $z$ and $1-z$, forming a colour dipole of transverse size $r \sim 1 / \sqrt{ } Q^{2}$. The dipole then scatters elastically from the proton according to a dipole cross section. The example shown corresponds to the amplitude for the total inclusive $\gamma^{*} p$ cross section. However, only small modifications are required to adapt this to the cases of exclusive or inclusive diffraction.

\section{B. Exclusive Production of $1^{--}$States}

\section{Kinematics and Experimental Selection}

Quasi-elastic vector meson production and DVCS (Fig. 45(a)) are the simplest diffractive processes that can be studied at HERA. For a fixed final state vector meson or photon, they are usually described in terms of the kinematic variables, $Q^{2}, W$ and $t$. Distributions in all three of these variables have been measured in analyses covering the vector meson species, $\rho, \omega, \phi, \rho^{\prime}, J / \psi$, $\psi^{\prime}$ and $\Upsilon$ as well as in DVCS. No evidence has been found for the exclusive production at the photon vertex of particles with non-1-- quantum numbers such as the $\pi^{0}$ Adloff et al., 2002f), as would be expected for the exchange of the postulated negative $C$-parity partner of the pomeron, the odderon (Lukaszuk and Nicolescu, 1973).

The most precise vector meson data are obtained by reconstructing two-prong decays via charged decay products (notably $\rho^{0} \rightarrow \pi^{+} \pi^{-}, \phi \rightarrow K^{+} K^{-}$and $J / \psi \rightarrow e^{+} e^{-}$ or $\mu^{+} \mu^{-}$) and requiring no further activity beyond the noise levels in the detector, except that associated with the scattered beam electron. DVCS selections require a similar lack of activity in the detector beyond the final state electron and photon, the main experimental complication being separating the signal from the competing purely electromagnetic Bethe-Heitler process, which generates identical final states. In contrast to inclusive diffractive studies, the simplicity of these final states and the high precision of the tracking detectors have allowed precision $t$ measurements without the need to tag the intact outgoing proton. This reconstruction method uses $\left|t-t_{\min }\right|=p_{T}^{2}(V)$, where the vector meson transverse momentum $p_{T}(V)$ is obtained from its decay products and $\left|t_{\text {min }}\right|$, the minimum kinematically accessible value of $|t|$, is usually negligible.

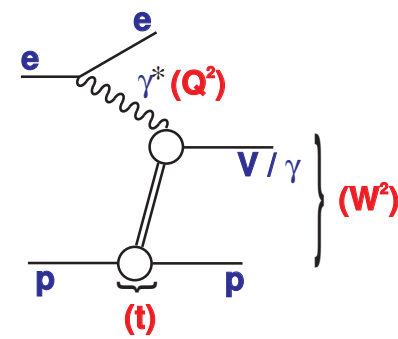

(a)

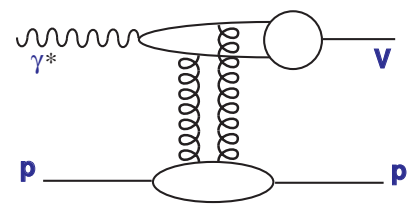

(b)
FIG. 45 (a) Generic representation of exclusive vector meson production via the quasi-elastic scattering of a real or virtual photon. The commonly used kinematic variables discussed in the text are indicated. (b) Lowest order perturbative diagram for hard vector meson production, involving the exchange of a pair of gluons between the proton and the real or virtual photon.

\section{General Characteristics of Vector Meson Production}

Vector meson production has emerged as a sensitive probe of the transition from the soft diffractive dynamics which are familiar from hadronic scattering experiments to a harder regime which may be calculated perturbatively. The former regime is encountered wherever no hard scale is present, the classic example being $\rho^{0}$ photoproduction (Aid et al., 1996c; Breitweg et al., 1998c, 2000a). Under such circumstances, the energy dependence of the photon-proton process is in good agreement with the form predicted by Regge asymptotics:

$$
\sigma^{\gamma p \rightarrow V p} \propto\left(W^{2}\right)^{2 \alpha_{\mathbb{P}}(t)-2}
$$

where the pomeron trajectory, $\alpha_{\mathrm{IP}}(t)=\alpha_{\mathbb{P}}(0)+\alpha_{\mathrm{PP}}^{\prime} t$, is assumed to be linear and its intercept $\alpha_{\mathrm{ID}}(0) \simeq 1.085$ (Cudell et al., 1997; Donnachie and Landshoff, 1992). This has been found to work well for $\rho^{0}$ photoproduction data, though interestingly, the slope of the pomeron trajectory has been found (Breitweg et al., 2000a) to be significantly smaller than the value of $\alpha_{\mathrm{m}}^{\prime} \sim 0.25$ (Abe et al., 1994; Donnachie and Landshoff, 1984) obtained from soft $p p$ and $p \bar{p}$ scattering. A possible explanation for this may be found in process-dependent absorptive corrections, which are absent in DIS, present to some extent in photoproduction and to a larger extent in fully hadronic scattering. Detailed models of these effects can be found for example in (Gotsman et al., 2007; Kaidalov et al., 2003, 2010). Further characteristics of this soft regime are (Aid et al., 1996c; Breitweg et al., 1998c) a skewed lineshape for the $\rho$ meson due to its interference with non-resonant $\pi^{+} \pi^{-}$production and a large value, $B \sim 10 \mathrm{GeV}^{-2}$, of the slope parameter de- 
scribing the $t$ dependence according to

$$
\frac{\mathrm{d} \sigma^{\gamma p \rightarrow V p}}{\mathrm{~d} t}=\left(\frac{\mathrm{d} \sigma^{\gamma p \rightarrow V p}}{\mathrm{~d} t}\right)_{t=0} e^{B t} .
$$

Interpreted in a simple optical model as a measurement of the mean impact parameter between the incoming photon and proton, these $B$ values suggest that the interaction takes place over a transverse distance of typically $1-1.5 \mathrm{fm}$.

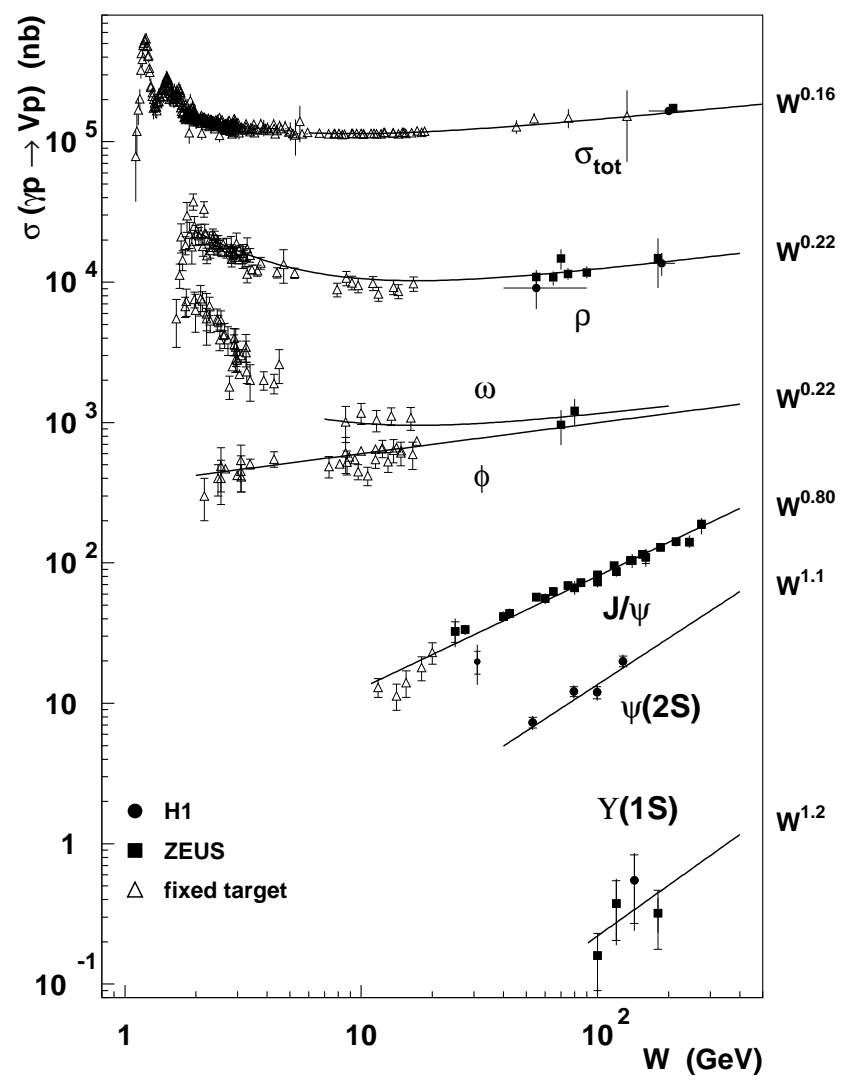

FIG. 46 Compilation of photoproduction cross section measurements as a function of the $\gamma p$ centre-of-mass energy, $W$. The total cross section and various vector meson production cross sections are included, with the approximate power law dependences $\sigma \propto W^{\delta}$ indicated for each process.

Wherever hard scales are present, usually provided either by heavy quarks in the vector meson or by large $Q^{2}$, but sometimes also by large $|t|$, the qualitative picture changes. The energy dependence becomes progressively steeper, such that the $W$ dependence, parametrised similarly to Eq. 9, yields an increased effective value of $\alpha_{\mathbb{P}}(0)$. This effect is illustrated in Fig. 46, where a compilation of photoproduction data on the total cross section and different vector meson production cross sections is shown as a function of $W$. The steepening of the dependence on $W$ for the heaviest vector mesons can be interpreted in terms of the scale dependence of the proton gluon density at low $x$, as discussed further below.

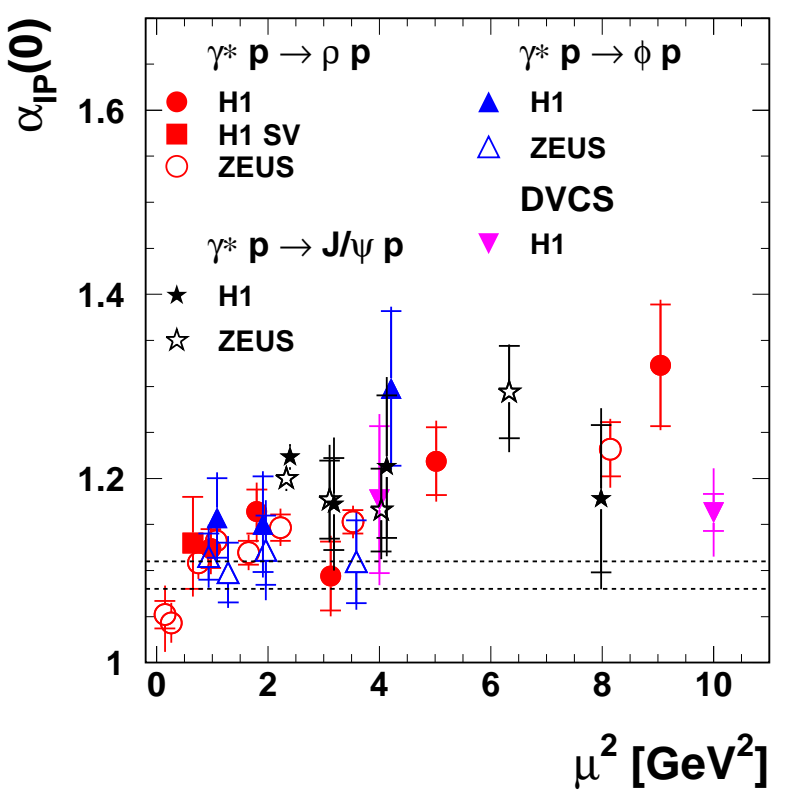

FIG. 47 Development of the effective pomeron intercept (see Eq. 9 and surrounding text) with a characteristic scale, $\mu^{2}=\left(Q^{2}+M_{V}^{2}\right) / 4$, derived from fits to the $W$ dependences of various vector meson production data, as well as a DVCS measurement (shown at $\mu^{2}=Q^{2}$ ). The dashed lines indicate the range of values which are typical in soft diffraction. From (Aaron et al., 2010b).

The transition from soft hadronic to perturbative behaviour is neatly mapped out in a single process in $\rho^{0}$ electroproduction data. As $Q^{2}$ increases, the $t$ slope parameter $B$ decreases, the $W$ dependence becomes steeper and the lineshape skewing disappears, all in a manner which has been measured with good precision (Aaron et al., 2010b; Adloff et al., 2000a; Breitweg et al., 1999a; Chekanov et al., 2007e). An example, also including comparisons with other exclusive vector meson production processes, is shown in Fig. 47. Here, the effective pomeron intercept (Eq. 9) is shown as a function of a scale, which is chosen to be $\left(Q^{2}+M_{V}^{2}\right) / 4$ (see Section V.B.5). As the scale increases, the effective pomeron intercept shifts from values typical of soft hadronic scattering to values which are compatible with results for the equivalent quantity $\alpha_{\mathrm{IP}}(0)=1+\lambda$ in fits of inclusive low- $x$ HERA data to the form $F_{2}\left(x, Q^{2}\right) \propto x^{-\lambda\left(Q^{2}\right)}$ (Adloff et al., 2001c; Chekanov et al., 2008b).

The exponential $t$ slopes of vector meson production processes are also found to vary systematically with scale and are approximately invariant in $Q^{2}+M_{V}^{2}$. A compendium of results is shown in Fig. 48. Although the uncertainties are often large and there is some scatter, the data suggest a convergence towards an asymptotic value of $B \sim 5 \mathrm{GeV}^{-2}$. In optical models, this can be interpreted as the point at which the physics is entirely short-distance in nature, the size of the probe becomes negligible and the slope parameter measures the 
size of the proton. Quantitatively, this indicates an effective proton size of around $0.6 \mathrm{fm}$, which is interestingly smaller than the value of $\sim 0.8 \mathrm{fm}$ which is well measured using electromagnetic probes. Interpreting vector meson production in terms of gluon exchange, this suggests that the gluon radius of the proton may be smaller than its quark radius.

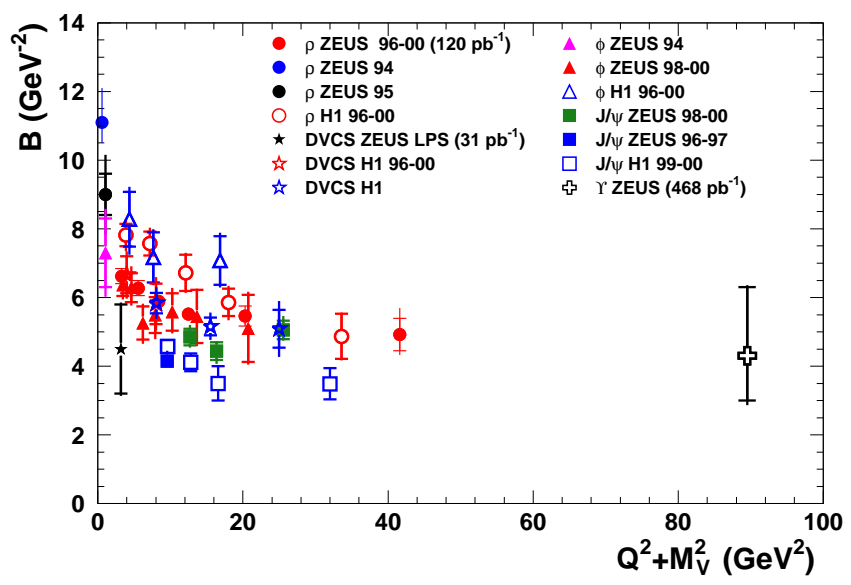

FIG. 48 Exponential $t$ slopes for vector meson electroproduction and photoproduction as a function of the characteristic scale $Q^{2}+M_{V}^{2}$. From (Abramowicz et al., 2012d).

\section{Vector Meson Production in QCD}

The observed relationship between the $W$ dependences of inclusive DIS and vector meson production in the presence of a sufficiently large scale encourages a perturbative approach. This has evolved considerably in the HERA era, to the point where most observables can be successfully predicted. Although the basic quark charge counting SU(4) prediction for the ratio of cross sections $\rho: \omega: \phi: J / \psi=9: 1: 2: 8$ holds approximately when viewed as a function of the scale $\left(Q^{2}+M_{V}^{2}\right) / 4$ (Aaron et al. , 2010b), vector meson wavefunction effects remain a significant source of uncertainty.

A QCD collinear factorisation theorem (Collins et al., 1997), valid for the leading power of $Q^{2}$ where $|t| \ll$ $\Lambda_{\mathrm{QCD}}^{2}$ relates cross sections for heavy vector meson production from longitudinally polarised photons to the generalised parton densities (GPDs) of the proton (Diehl, 2003) (see Section V.B.4). Neglecting skewing effects, in which the two exchanged partons carry different fractions of the proton longitudinal momentum, the GPDs reduce to the square of the gluon density of the proton. The process is then driven to first approximation by the exchange of a pair of gluons from the proton structure, as illustrated in Fig. 45(b). Later approaches (Martin et al., 1997) incorporated transversely polarised photon cross sections at sufficiently large $Q^{2}$ and light vector mesons in a similar framework, with some degree of success.
Since it is relatively uncomplicated theoretically, has a reasonably large scale, probes small $x$ values and is experimentally clean, $J / \psi$ photoproduction is an ideal testing ground for these ideas and, with sufficiently strong theoretical understanding, a potentially competitive means of extracting the gluon density of the proton. The many measurements at HERA (Aktas et al., 2006c; Alexa et al., 2013a; Chekanov et al., 2002c) have recently been supplemented by the first measurements from ultra-peripheral processes at the LHC, extending the $W$ range to beyond $1 \mathrm{TeV}$ for the forward kinematics of LHCb (Aaij et al., 2013). The $W$ dependence of the cross section for $J / \psi$ photoproduction has been calculated using the proton gluon density by a number of groups (Frankfurt et al., 2001b; Martin et al., 2008, 2000). An example comparison with data is shown in Fig. 49, From comparisons between the predictions with different gluon densities, it is clear that there is high sensitivity. However, the normalisation is not well predicted and there remains considerable model dependence.
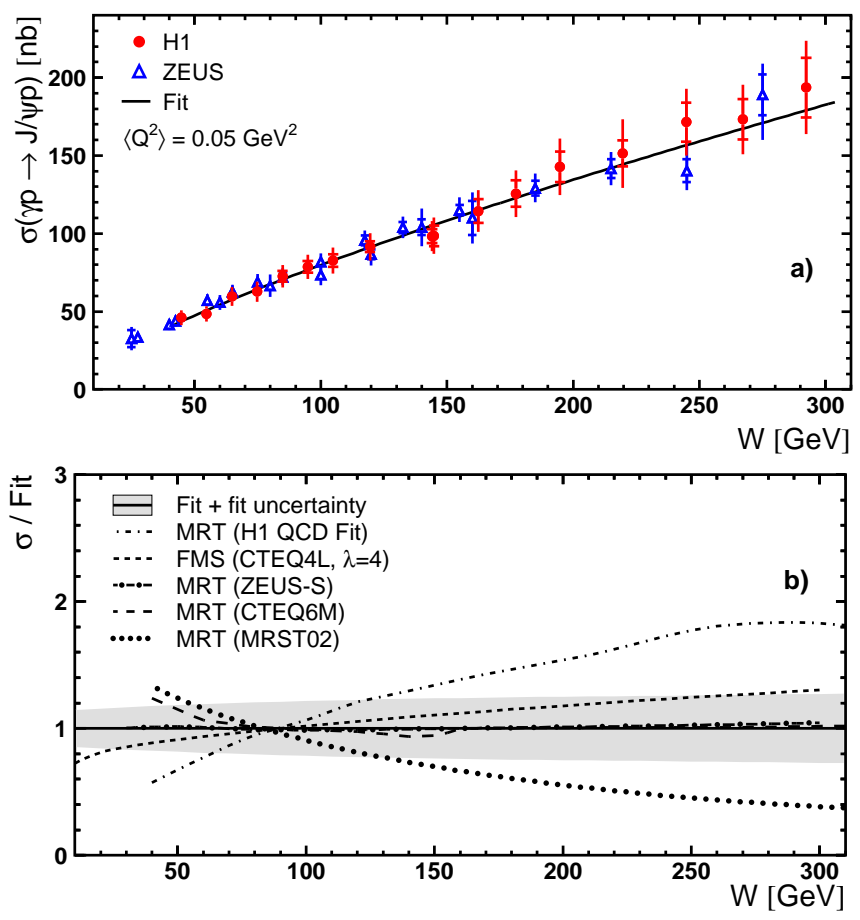

FIG. $49 J / \psi$ photoproduction cross section as a function of $W$, compared with a QCD calculation, MRT (Martin et al., 2000), based on four different parametrisations of the gluon density of the proton and a dipole model, FMS (Frankfurt et al., 2001b). The predictions are normalised to the data. From (Aktas et al., 2006c).

More detailed comparisons with QCD models require an understanding of the helicity structure of vector meson production. The five independent helicity amplitudes, describing transitions from either longitudinally or transversely polarised photons to either longitudinally or transversely polarised vector mesons, can be ex- 
tracted from the production and decay angular distributions via spin density matrix elements as parametrised in (Schilling and Wolf, 1973). Whilst helicity amplitudes for $J / \psi$ production at modest $|t|$ are consistent with $s$-channel helicity conservation (Aktas et al., 2006c), significant 'helicity flip' amplitudes, for which the vector meson helicity differs from that of the photon, are observed for the case of $\rho$ production (Aaron et al., 2010b; Adloff et al., 2002a; Breitweg et al., 2000c; Chekanov et al., 2007e) and for $J / \psi$ production at large $|t|$ (Aktas et al., 2003; Chekanov et al., 2010f). This reflects the often unequal sharing of the longitudinal momentum between the two quarks, corresponding to values of $z$ close to 0 or 1 in Fig. 44, particularly for the transverse polarisation case with $\rho$ mesons, This allows the helicity to flip at sufficiently large $t$, an effect which can be reasonably well predicted in QCD-based models.

Probably the most important feature of angular distribution analyses is that they allow an unfolding of the cross sections $\sigma_{L}$ and $\sigma_{T}$, corresponding to vector meson production from longitudinally and transversely polarised vector mesons, respectively. The $Q^{2}$ dependences of $\sigma_{L}$ and $\sigma_{T}$ are separately predicted by QCD models. The basic leading order dependences at $t=0$ of $\sigma_{L} \propto 1 / Q^{6}$ and $\sigma_{T} \propto 1 / Q^{8}$ (Brodsky et al., 1994) are expected to be strongly violated, in particular due to the $Q^{2}$ dependence of the proton gluon density, but also due to the quark virtuality and the running of $\alpha_{s}$ (Frankfurt et al., 1996). The HERA data for all vector meson species exhibit approximate scaling with power law dependences of approximately $\sigma_{L} \propto\left(Q^{2}+M_{V}^{2}\right)^{2.1}$ and $\sigma_{T} \propto\left(Q^{2}+M_{V}^{2}\right)^{2.9}$, which are reasonably well reproduced by both collinear factorisation and dipole-based models (Kowalski et al., 2006; Martin et al., 1997).

As discussed in Section V.B.2 the variation of the slope parameter $B$ with centre-of-mass energy $W$ is usually parametrised in terms of the slope of the pomeron trajectory $\alpha_{\mathbb{P}}^{\prime}$. Values close to zero are measured with small uncertainties in some hard exclusive processes, notably $J / \psi$ production at large $|t|$ (Aktas et al., 2006c; Chekanov et al., 2002d, 2004d). This is consistent with expectations in the BFKL approach (Brodsky et al., 1999; Nikolaev et al., 1996), where $\alpha_{\mathrm{IP}}^{\prime}$ is related to the average transverse momentum of partons along the gluon ladder. However, it is hard to draw quantitative conclusions in light of the smaller-than-expected $\alpha_{\mathbb{I P}}^{\prime}$ values for soft processes such as $\rho^{0}$ photoproduction (Section V.B.2.

\section{Deeply Virtual Compton Scattering and Generalised Parton Densities}

Due to the underlying exchange of a pair of gluons, which in general differ in both longitudinal and transverse momentum, hard exclusive DIS processes have emerged in recent years as candidates with which to understand correlations between partons in the proton and thus the transverse spatial, momentum and spin distributions of partons. This information is encoded in GPDs (Diehl, 2003), which were introduced in Section V.B.3. HERA data offer unique sensitivity to GPDs at low $x$. This topic is most commonly associated with the DVCS process, though it is also relevant to vector meson production.

The dominant underlying parton level process for DVCS at low $x$ is similar to that shown in Fig. 45(b), though there is also a 'handbag' contribution in which the incoming (virtual) and outgoing (real) photons couple directly to quarks from the GPDs. DVCS has the advantage of avoiding the complication of the vector meson wavefunction, but cross sections are suppressed due to the final state photon coupling. Due to the smaller cross sections compared with their vector meson counterparts, DVCS studies emerged relatively late in the lifetime of HERA. For the HERA kinematic region, integrated over azimuthal degrees of freedom, interference between DVCS and the competing Bethe-Heitler process is small and DVCS cross sections can be extracted by statistically subtracting the Bethe-Heitler component using Monte Carlo models tuned in regions in which the DVCS contribution can be neglected.

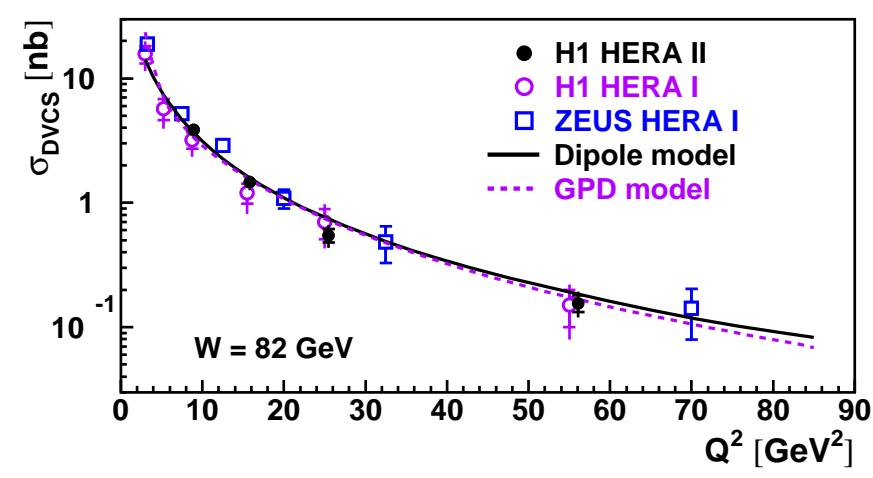

FIG. 50 Dependence of HERA DVCS cross section measurements on $Q^{2}$. The data are compared with a GPD-based model (Kumericki and Mueller, 2010) and also with a dipolebased model (Marquet et al., 2007). See SectionV.B.3 for an explanation of the latter. From (Aaron et al., 2009c).

By the end of HERA operation, $Q^{2}, W$ and $t$ distributions had all been measured with good precision (Aaron et al., 2008a, 2009c; Chekanov et al., 2009a). As shown in Figs. 47 and 48 , the exponential $t$ slope is $B \simeq 5 \mathrm{GeV}^{-2}$ and parametrisations of the cross section in the usual $W^{\delta}$ form yield $\delta \approx 0.5-0.6$. Example $Q^{2}$ dependence data are shown in Fig. 50. The DVCS cross section falls more slowly (roughly as $\left(Q^{2}\right)^{-1.5}$ ) with photon virtuality than is the case for vector meson production. This observation is in line with theoretical predictions (Frankfurt et al., 1998) and is partially explained by the presence of the quark-driven handbag contribution, which is not relevant to vector meson production. Together, these observations indicate that DVCS can be considered as a hard process, even at relatively small $Q^{2}$. 
Asymmetries in the azimuthal degree of freedom between the lepton and the (virtual-to-real) photon scattering planes in DVCS give access to the real part of the amplitude, and are most sensitive to the non-diagonal GPDs. The first $\mathrm{H} 1$ data have appeared on the DVCS beam charge asymmetry (Aaron et al., 2009c). It is clearly non-zero and its azimuthal dependence has been used to confirm that the DVCS amplitude is dominantly imaginary for HERA kinematics. It has not yet been possible to determine beam polarisation asymmetries in the manner successfully pursued in the fixed target configuration by HERMES (Airapetian et al., 2012a, b). Nonetheless, the $\mathrm{H} 1$ and ZEUS data will provide essential low $x$ ingredients in models of GPDs for a long time to come (Kumericki and Mueller, 2010), the principal sensitivity being to the GPD, $H$.

\section{Exclusive Processes in Dipole Models and Low $\boldsymbol{x}$ Saturation}

Viewed in the proton rest frame, vector meson production naturally factorises into three separate processes, occurring on distinct time-scales (c.f. Figs. 44 and 45 (b)). The incoming real or virtual photon first fluctuates into a $q \bar{q}$ pair, which creates a colour dipole of transverse size $r_{\perp} \sim 1 / \sqrt{ } Q^{2}$ (Wusthoff, 1997). The dipole then scatters elastically from the proton, a process which is generically described in terms of a dipole cross section. Finally, the outgoing colour dipole combines back to a photon in the DVCS case, or else hadronises to a final state vector meson in a manner which depends on the non-perturbative vector meson wavefunction. The scale choice of $\left(Q^{2}+M_{V}^{2}\right) / 4$, already used in the previous sections, corresponds to the inverse of the scanning radius of the dipole-proton interaction, for the case where the incoming photon longitudinal momentum is shared equally between the quark and antiquark forming the dipole (Frankfurt et al., 1996; Ivanov et al., 2006). It is appropriate for heavy vector mesons and for light vector mesons produced by longitudinally polarised photons.

At small enough $r_{\perp}$, the dipole cross section can be described perturbatively in the manner discussed in Section V.B.3. At larger $r_{\perp}$, the dipole cross section is not known a priori, but is sensitive to any low $x$ saturation of the proton parton densities. There are various parametrisations of the dipole cross section which include saturation phenomena, some of which are based on particular approximations to QCD with non-linear evolution such as the Colour Glass Condensate (Iancu et al., 2004) and some of which are purely phenomenological (Forshaw et al., 1999, 2004; Golec-Biernat and Wusthoff, 1998, 1999).

In the most sophisticated dipole models of vector meson production, the target matter density, and hence any saturation effects, depend on the distance from the centre of the proton, such that the dipole cross section varies with impact parameter (Kowalski et al., 2006; Kowalski and Teaney, 2003; Rezaeian et al., 2013), which in turn is closely related to $1 / t$ (see also (Marquet et al., 2007) for a complementary approach). When viewed differentially in $t$, the influence of the saturation effects thus becomes stronger as $t$ increases (Abelleira Fernandez et al., 2012; Kowalski et al., 2006). Such models adequately describe almost all aspects of vector meson production and DVCS at HERA. An example comparison with the inclusive $J / \psi$ photoproduction cross section as a function of $W$, also illustrating the predicted size of the saturation effects, is shown in Fig. 51 .

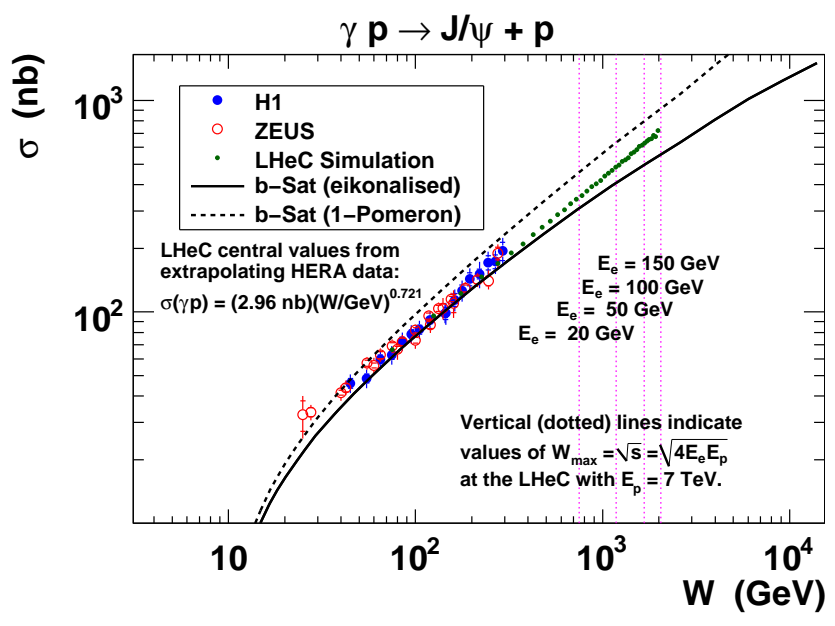

FIG. 51 Exclusive $J / \psi$ photoproduction data compared with versions of the b-Sat dipole model (Kowalski et al., 2006) both with (eikonalised) and without (1-Pomeron) saturation effects included. An extrapolation into the kinematic regime of a possible future ep collider is also included. Figure from (Abelleira Fernandez et al., 2012).

\section{Inclusive Diffractive Dissociation}

\section{Kinematics and Experimental Selection of Diffraction}

The kinematic variables describing the inclusive diffractive DIS (DDIS) process $e p \rightarrow e X p$ are illustrated in Fig. $52(\mathrm{a})$. In addition to $x, Q^{2}$ and $t(<0)$, there is one further non-trivial invariant, corresponding to the mass $M_{X}$ of the diffractive final state. In practice, the variable $M_{X}$ is usually expressed in terms of

$$
\beta=\frac{Q^{2}}{Q^{2}+M_{X}^{2}-t} .
$$

Small values of $\beta$ thus refer to events with diffractive masses much bigger than the photon virtuality, while exclusive processes have values of $\beta$ close to unity. In models based on a factorisable pomeron (Section V.C.3), $\beta$ may be interpreted as the fraction of the pomeron longitudinal momentum which is carried by the struck parton. The longitudinal momentum fraction of the colourless exchange with respect to the incoming proton is denoted $x_{\mathbb{P}}$, such that $\beta x_{\mathbb{P}}=x$. 

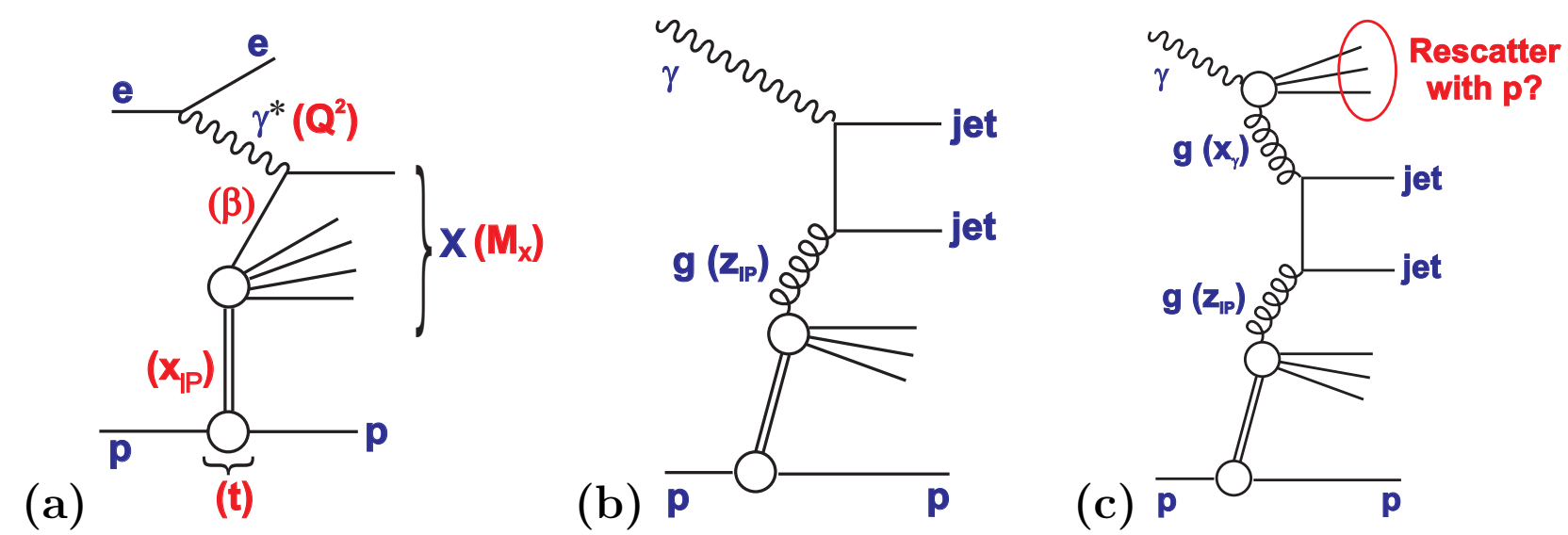

FIG. 52 Sketches of diffractive ep processes. (a) Inclusive DDIS at the level of the quark parton model, illustrating the kinematic variables discussed in the text. (b) Dominant leading order QCD diagram for hard scattering in DDIS or direct photoproduction, in which a parton of momentum fraction $z_{\mathbb{P}}$ from the DPDFs enters the hard scattering. (c) A leading order process in resolved photoproduction involving a parton of momentum fraction $x_{\gamma}$ relative to the photon.

Experimentally, diffractive ep scattering is characterised by the presence of a leading proton in the final state, retaining most of the initial state proton energy, and by a lack of any other hadronic activity in the forward (outgoing proton) direction, such that the system $X$ is cleanly separated and $M_{X}$ may be measured in the central detector components. These signatures have been widely exploited at HERA to select diffractive events by tagging the outgoing proton in the H1 Forward Proton Spectrometer or the ZEUS Leading Proton Spectrometer (proton-tagging method, see Fig. 7) or by requiring the presence of a large gap in the rapidity distribution of hadronic final state particles in the forward region (LRG method). In a third approach ( $M_{X}$ method (Breitweg et al., 1999e; Chekanov et al., 2005g, 2008b)), the inclusive DIS sample is decomposed into diffractive and non-diffractive contributions based on their characteristic dependences on $M_{X}$. Whilst the LRG and $M_{X}$-based techniques yield better statistics than the LPS method, they suffer from systematic uncertainties associated with an admixture of proton dissociation to baryon states with small masses, typically $M_{Y} \lesssim 1.6 \mathrm{GeV}$, which is irreducible due to the limited forward detector acceptance. Detailed comparisons between cross sections obtained by the LRG and proton tagging methods can be found in (Aaron et al., 2011c; Chekanov et al., 2009b). Neither collaboration observes any evidence for deviations from a constant ratio of cross sections measured by the two methods, indicating that the ratio of probabilities for the proton to scatter elastically and to undergo a low mass excitation is independent of the inclusive kinematic quantities.

\section{Inclusive Diffraction Data}

Observations of diffractive dissociation in DIS (Ahmed et al., 1994a; Derrick et al., 1993) and of jet production in diffractive photoproduction Ahmed et al., 1995b; Derrick et al., 1995d) and DIS (Derrick et al., 1994b) were among the earliest HERA publications, based on rapidity gaps which were much larger than could conceivably occur at significant rates in standard fragmentation models (Andersson et al., 1983). As increasingly larger datasets became available, the precision on the inclusive diffraction cross sections improved correspondingly, until the final measurements covered three-fold differential cross sections with a precision of a few percent (Aaron et al., 2012b; Chekanov et al., 2009b) over large phase space regions.

In analyses of data with protons tagged in the LPS and FPS, the $t$ dependence of DDIS has been measured and, as in the case of exclusive vector meson production, found to be compatible with an exponential parametrisation of the form of Eq 10. Example H1 data on the slope parameter $B$ are shown in Fig. 53 (Aaron et al., 2011c). Similar results, with globally slightly larger $B$ values, are obtained by ZEUS (Chekanov et al., 2009b). Beyond the low $M_{X}$ resonance region, $B$ is typically around $6-7 \mathrm{GeV}^{-2}$, independently of $\beta$ and $Q^{2}$, but with some dependence on $x_{\mathbb{P}}$, which is further discussed in Section V.C.4 This is indicative of an approximate factorisation of the proton vertex from the virtual photon vertex in DDIS (Section V.C.3).

The semi-inclusive DDIS cross section is usually presented in the form of a diffractive reduced cross section $\sigma_{r}^{D(3)}$, related to the experimentally measured differential cross section by

$$
\frac{\mathrm{d}^{3} \sigma^{e p \rightarrow e X p}}{\mathrm{~d} x_{\mathbb{P}} \mathrm{d} x \mathrm{~d} Q^{2}}=\frac{2 \pi \alpha^{2}}{x Q^{4}} \cdot Y_{+} \cdot \sigma_{r}^{D(3)}\left(x_{\mathbb{P}}, x, Q^{2}\right)
$$

where $Y_{+}=1+(1-y)^{2}$ and $y$ is the usual Bjorken variable. The reduced cross section depends at moderate scales, $Q^{2}$, on two diffractive structure functions $F_{2}^{D(3)}$ 


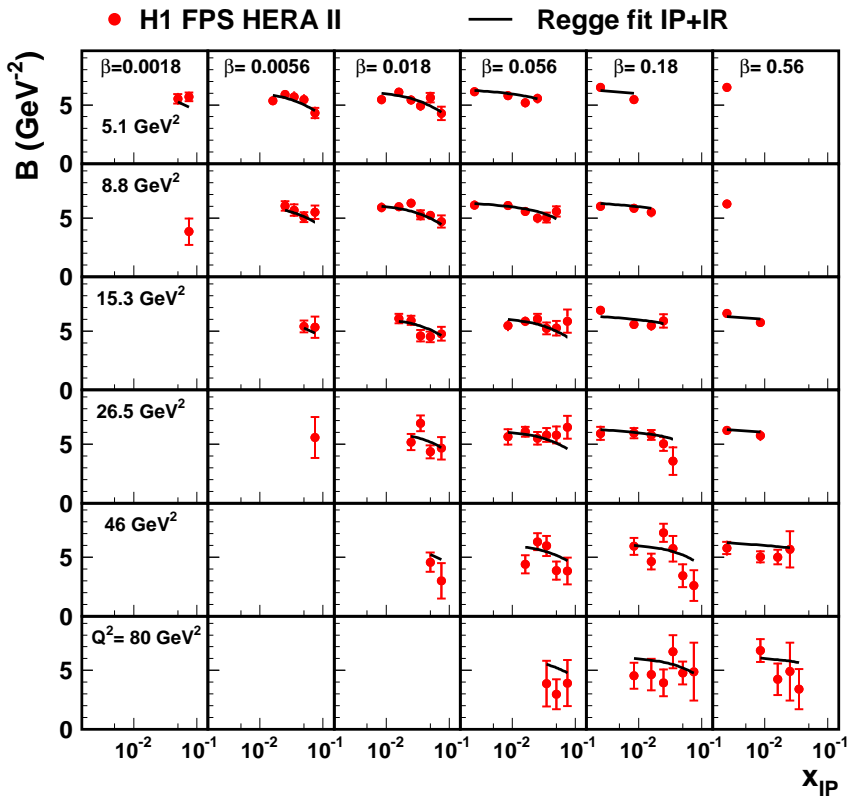

FIG. 53 Example data on the dynamics of the slope parameter, $B$, describing the $t$ dependence of DDIS, as measured using the H1 FPS. From (Aaron et al., 2011c).

and $F_{L}^{D(3)}$ according to

$$
\sigma_{r}^{D(3)}=F_{2}^{D(3)}-\frac{y^{2}}{Y_{+}} F_{L}^{D(3)} .
$$

For $y$ not too close to unity, $\sigma_{r}^{D(3)}=F_{2}^{D(3)}$ holds to very good approximation. Measurements have also been made of the full four dimensional reduced cross section, dependent also on $t$ (Aaron et al., 2011c; Chekanov et al., 2009b). However, since the $t$ dependence factorises to good approximation, this degree of freedom is usually integrated out. A more detailed exposition of the formalism of diffractive DIS can be found for example in (Aktas et al., 2006e).

With the most recent $\sigma_{r}^{D}$ data, close agreement has developed between the H1 and ZEUS measurements. A first combination of data from the two experiments, using the proton tagging method, has recently been published (Aaron et al., 2012a). The cross-calibration of the systematic uncertainties between the two experiments implicit in the averaging procedure leads to an improvement in precision well beyond that expected from statistical considerations alone. Full combinations of data from the two collaborations for the LRG method have yet to be performed, mainly due to some non-trivial discrepancies, usually at the edges of the accessible phase space. An investigation of the residual differences and crude combination may be found in (Newman and Ruspa, 2009). The comparison of final data in the most precise region is illustrated in Fig. 54. Whilst there is good agreement in the bulk of the phase space, residual disagreements are apparent at small $\beta$ and small $x_{\mathbb{P}}$. These discrepancies have thus far prevented a combination of the $\mathrm{H} 1$ and
ZEUS data, though they have only a small influence on the interpretation of the data discussed in the following.

Various models have been used to interpret DDIS data. Since it has become standard, Sections V.C.3 V.C.7 focus mainly on the 'proton vertex factorisation' approach, in which the diffractive exchange ('pomeron') has a resolved structure. The relevance of DDIS to dipole models is discussed separately in Section V.C.8. It is worth noting that a third approach, in which rapidity gaps are randomly produced from final state colour interactions in otherwise standard DIS events, has been at least partially successful. This model is attractive in its simplicity. Indeed an early incarnation (Buchmuller and Hebecker, 1995) in which diffractive final states simply emerge randomly due to colour rotations in one ninth of all low $x$ DIS events works remarkably well for inclusive data. Closely related ideas are encoded in the 'Semi-classical model' (Buchmuller et al., 1999, 1997). The 'Soft Colour Interaction' model (Edin et al., 1996) offers a rather different, but again related, approach, which later developed into the 'Generalised Area Law' (Rathsman, 1999) model. All of these approaches have enjoyed success, particularly in comparisons with $F_{2}^{D}$ and $\sigma_{r}^{D}$ data, but none provides a comprehensive description, breaking down for example when confronted with more exclusive processes such as diffractive dijet production (Adloff et al., 2001a).

\section{Factorisation Studies}

Cleanly interpreting a three (or even four)-fold differential cross section is far from easy. It is simplified considerably if the dependences on the relevant kinematic variables can be factorised. QCD hard scattering collinear factorisation, when applied to diffractive DIS (Collins, 1998), implies that the cross section for the process $e p \rightarrow e X Y$ can be written in terms of convolutions of partonic cross sections $\hat{\sigma}^{e i}\left(x, Q^{2}\right)$ with diffractive parton density functions (DPDFs) $f_{i}^{D}$ as

$$
\begin{aligned}
\mathrm{d} \sigma^{e p \rightarrow e X Y}\left(x, Q^{2}, x_{\mathbb{P}}, t\right)= & \sum_{i} f_{i}^{D}\left(x, Q^{2}, x_{\mathbb{P P}}, t\right) \\
& \otimes \mathrm{d} \hat{\sigma}^{e i}\left(x, Q^{2}\right),
\end{aligned}
$$

with additional implicit dependences on $\mu_{F}, \mu_{R}$ and $\alpha_{S}$ as in Eq 1. The partonic cross sections are the same as those for inclusive DIS. This factorisation formula is valid for sufficiently large $Q^{2}$ and fixed $x_{\mathbb{P}}$ and $t$.

The experimental data over most of the accessible phase space are compatible with the deeper 'proton vertex' factorisation (Ingelman and Schlein, 1985), in which the DPDFs may be factorised into a term containing only variables associated with the virtual photon vertex $(\beta$, $Q^{2}$ ) and a term containing variables associated with the proton vertex $\left(x_{\mathbb{I P}}, t\right)$ :

$$
f_{i}^{D}\left(x, Q^{2}, x_{\mathbb{P}}, t\right)=f_{\mathbb{P} / p}\left(x_{\mathbb{P}}, t\right) \cdot f_{i}\left(\beta=x / x_{\mathbb{P}}, Q^{2}\right) .
$$

This is equivalent to considering the diffractive exchange as a 'pomeron' with a partonic structure given by the par- 

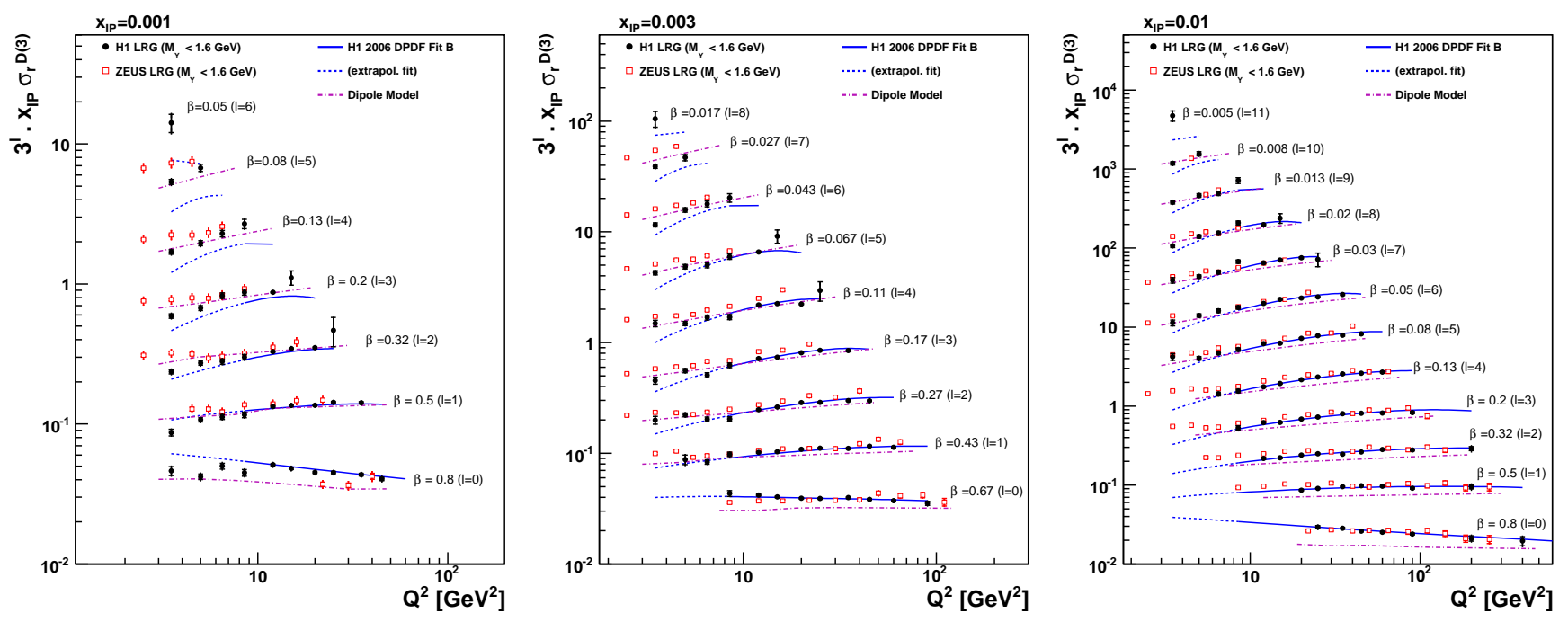

FIG. 54 Comparisons between H1 and ZEUS DDIS data measured using the LRG method at $x_{\mathrm{IP}}=0.001, x_{\mathrm{IP}}=0.003$ and $x_{\mathrm{IP}}=0.01$. From (Aaron et al., 2012b).

ton distributions $f_{i}\left(\beta, Q^{2}\right)$, the variable $\beta$ corresponding to the fraction of the pomeron longitudinal momentum carried by the struck quark. The 'pomeron flux factor' $f_{\mathbb{P} / p}\left(x_{\mathbb{P}}, t\right)$ represents the probability that a pomeron with particular values of $x_{\mathbb{P}}$ and $t$ couples to the proton. Whilst this approach cannot be valid to ultimate precision (e.g. it is clearly violated in the high $\beta$ region where higher twist processes such as exclusive vector meson production play an important role), it is justified theoretically for the leading twist case (Blumlein and Robaschik, 2001).

As was first shown in (Adloff et al., 1997d), a good description of the data at relatively large $x_{\mathbb{P}}$ cannot be obtained by considering pomeron exchange alone. As in the case of total hadronic cross sections (Donnachie and Landshoff, 1992), sub-leading exchanges become important away from the asymptotic $\left(x_{\mathbb{I P}} \rightarrow 0\right.$ in this case) limit. An additional sub-leading exchange $(\mathbb{R})$ is therefore usually considered in addition, contributing at low $x_{\mathbb{P}}$ and $\beta$ and exhibiting a similar factorisation to the pomeron term, such that Eq. (15) is modified to

$$
\begin{aligned}
f_{i}^{D}\left(x, Q^{2}, x_{\mathbb{P}}, t\right) & =f_{\mathbb{P} / p}\left(x_{\mathbb{P}}, t\right) \cdot f_{i}\left(\beta, Q^{2}\right) \\
& +n_{\mathbb{R}} \cdot f_{\mathbb{R} / p}\left(x_{\mathbb{P}}, t\right) \cdot f_{i}^{\mathbb{R}}\left(\beta, Q^{2}\right),
\end{aligned}
$$

where $n_{\mathbb{R}}$ sets the relative normalisation of the subleading term. Further investigations of the sub-leading trajectory contribution can be found in Section V.D.

With the above ansatz, a rather complete description is obtained of all HERA inclusive diffractive data. The energy dependence of DDIS is encoded in the pomeron flux factor, which is parametrised based on Regge phenomenology, as discussed further in Section V.C.4 The DPDFs are treated in a similar way to the case of inclusive DIS, as described in Section V.C.5 Full details of the standard fitting scheme adopted for the most recent data by both collaborations can be found in Aktas et al., 2006a e). An improved heavy flavour treatment is described in (Chekanov et al., 2010a).

\section{Energy Dependences and Soft Phenomenology}

In the fits to DDIS data described in Section V.C.3, the $x_{\mathbb{P}}$ and $t$ dependences are parametrised using a flux factor motivated by Regge theory,

$$
f_{\mathbb{P} / p}\left(x_{\mathbb{P}}, t\right)=A_{\mathbb{P}} \cdot \frac{e^{B_{\mathbb{P}} t}}{x_{\mathbb{P}}^{2 \alpha_{\mathbb{P}}(t)-1}} .
$$

The parameters $B_{\mathbb{P}}$ and $\alpha_{\mathbb{P}}^{\prime}$ and their uncertainties are obtained from fits to FPS or LPS data Aktas et al., 2006a), which also take sub-leading contributions into account. The $x_{\mathbb{I P}}$ dependence of the data principally determines the effective pomeron intercept $\alpha_{\mathbb{P}}(0)$, appropriate to the DDIS data. The most precise determinations are obtained from LRG data, with remarkable consistency between statistically independent data sets:

$$
\begin{aligned}
& \alpha_{\mathbb{I P}}(0)=1.118 \pm 0.008(\text { exp. })_{-0.010}^{+0.029}(\text { model }) \\
& \text { (Aktas et al., 2006e) ; } \\
& \left.\alpha_{\mathbb{I P}}(0)=1.117 \pm 0.006 \text { (exp. }\right)_{-0.007}^{+0.022} \text { (model) } \\
& \text { (Chekanov et al., 2009b); } \\
& \left.\alpha_{\mathbb{P}}(0)=1.113 \pm 0.002 \text { (exp. }\right)_{-0.015}^{+0.029}(\text { model }) \\
& \text { (Aaron et al., 2012b), }
\end{aligned}
$$

the model dependence uncertainties being highly correlated between the different data sets.

These $\alpha_{\mathbb{I P}}(0)$ values are only slightly larger than their counterparts obtained from fits to total (Cudell et al., 1997; Donnachie and Landshoff, 1992) and diffractive 
(Aad et al., 2012b; Goulianos, 1983) cross sections in $p p$ and $p \bar{p}$ scattering and are compatible with results from soft photoproduction at HERA (Adloff et al., 1997b; Breitweg et al., 1997d). They are significantly smaller than the results obtained from the energy dependences of hard exclusive processes at HERA (Section V.B.2) and are much smaller than values predicted based on leading-logarithmic BFKL (Balitsky and Lipatov, 1978; Kuraev et al., 1976, 1977) or other 'hard pomeron' (Donnachie and Landshoff, 1998) approaches. This supports the picture of the dominating process in DDIS being the application of a hard virtual photon probe to an approximately factorisable exchange object, closely related to the pomeron of soft hadronic scattering.

The slope of the effective DDIS pomeron trajectory can be determined from the $x_{\mathbb{P}}$ dependence of the slope parameter $B$, as obtained using the LPS and FPS detectors. In this case, the best values obtained to date are:

$$
\begin{gathered}
\alpha_{\mathbb{P}}^{\prime}=-0.01 \pm 0.06(\text { stat. })_{-0.08}^{+0.04} \text { (syst.) } \pm 0.04 \text { (model) } \\
\alpha_{\mathbb{I P}}^{(\text {Chekanov et al. },, 2009 \mathrm{~b})} ; \\
\text { (Aaron et al. }, 2011 \mathrm{c}) .
\end{gathered}
$$

Whilst the precision on these results is limited, the trajectory slope is clearly smaller than the canonical value from soft $p p$ and $p \bar{p}$ scattering of $\alpha_{\mathrm{m}}^{\prime} \sim 0.25$ (Abe et al., 1994; Donnachie and Landshoff, 1984). As discussed in Section V.B.2, soft vector meson photoproduction data also show lower $\alpha_{\mathbb{P}}^{\prime}$ values than those usually taken from $p p$ and $p \bar{p}$ scattering (Breitweg et al., 2000a), suggesting that this is a heavily process dependent parameter, which is highly sensitive to absorptive corrections.

The validity of the factorisation assumption implicit in the fits to inclusive diffractive data is tested by adding further free parameters, which allow the pomeron intercept and the slope parameter $B$ to vary freely between different $Q^{2}$ or $\beta$ bins. A summary of ZEUS results on possible variations of $\alpha_{\mathrm{IP}}(0)$ with $Q^{2}$ is shown in Fig. 55. Both here and in corresponding $\mathrm{H} 1$ studies (see e.g. (Aaron et al., 2012b)), there is no evidence for any variation of the effective pomeron intercept with $Q^{2}$ or $\beta$ when considering data taken using the LRG or LPS/FPS methods. Similar searches for a $Q^{2}$ or $\beta$ dependence of $\alpha_{\mathrm{m}}^{\prime}$ or $B$ have also yielded null results (Aaron et al., 2011c), albeit with lesser precision than for the $\alpha_{\mathbb{P}}(0)$ case.

It is informative to compare the photon-proton centreof-mass energy ( $W$ or equivalently $1 / x$ at fixed $Q^{2}$ ) dependences of the diffractive and the inclusive cross sections. Basic Regge pole phenomenology predicts that the growth with centre-of-mass energy of the diffractive cross section $\left(\sim\left(W^{2}\right)^{2 \alpha_{\mathbb{P}}(t)-2}\right.$ at fixed $Q^{2}$ and $\beta$, with $\left.\langle t\rangle \sim 1 / B \sim 0.2 \mathrm{GeV}^{2}\right)$ should be faster than that of the total cross section $\left(\sim\left(W^{2}\right)^{\alpha_{\mathbb{P}}(0)-1}\right)$. However, in numerous studies of HERA data, the ratio of diffractive to inclusive cross sections has been found to be relatively flat

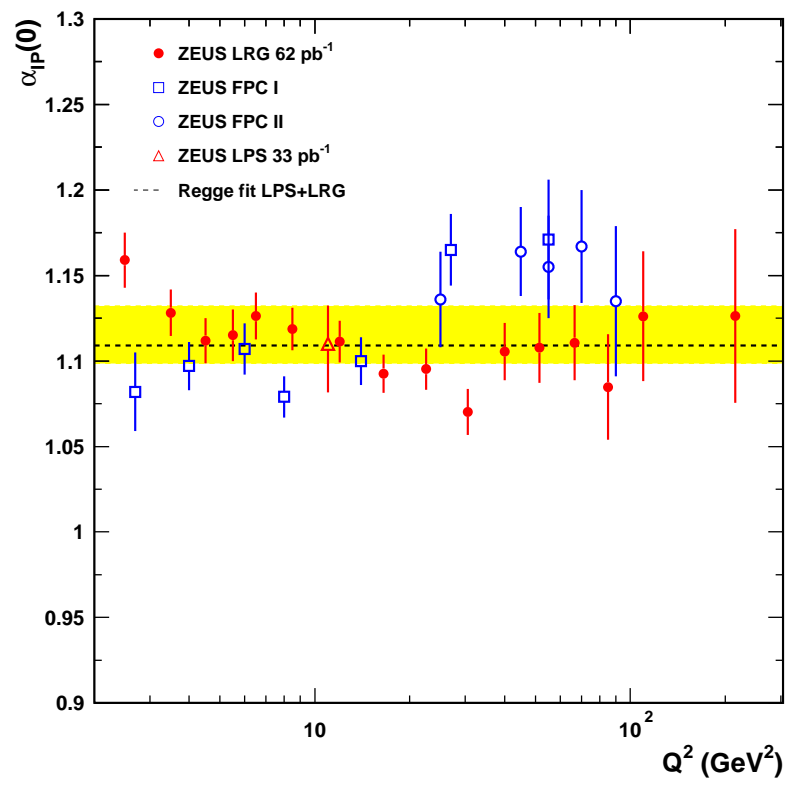

FIG. 55 Summary of ZEUS data on tests of proton vertex factorisation in the form of possible $Q^{2}$ variations of the pomeron intercept in fits to LRG and LPS data, as well as 'FPC' data obtained by the $M_{X}$ decomposition method. From (Chekanov et al., 2009b).

as a function of $W$. The first example (Breitweg et al., 1999e) is shown in Fig. 56. See also (Aaron et al., 2012b; Aktas et al., 2006e) for more recent results. This flatness represents a clear breakdown of the simple Regge approach, showing conclusively that there is no universal pomeron in virtual photon-proton scattering, as already discussed in Section V.B.2. The flatness of the ratio has been interpreted (Golec-Biernat and Wusthoff, 1998, 1999) as evidence for saturation of low $x$ parton densities, which is expected to be visible in diffraction at higher $x$ than in the inclusive case, due to the exchange of two, rather than one, gluon in the simplest interpretation. Any saturation effects thus tend to reduce the diffractive cross section relative to the inclusive in a manner which becomes more important as $x$ falls or $W$ grows. Whilst this is a highly suggestive observation, it has also been explained without invoking saturation, for example in models which explain rapidity gap formation as a random process, due to soft colour rearrangements (Buchmuller et al., 1999; Edin et al., 1996).

\section{QCD Phenomenology and Diffractive Parton Densities}

A cursory glance at Fig. 54 immediately leads to the conclusion that the scaling violations in diffraction remain of positive sign $\left(\partial \sigma_{r}^{D} / \partial Q^{2}>0\right)$ up to large values of $\beta \sim 0.5$, which may be compared with $x \sim 0.1$ for 


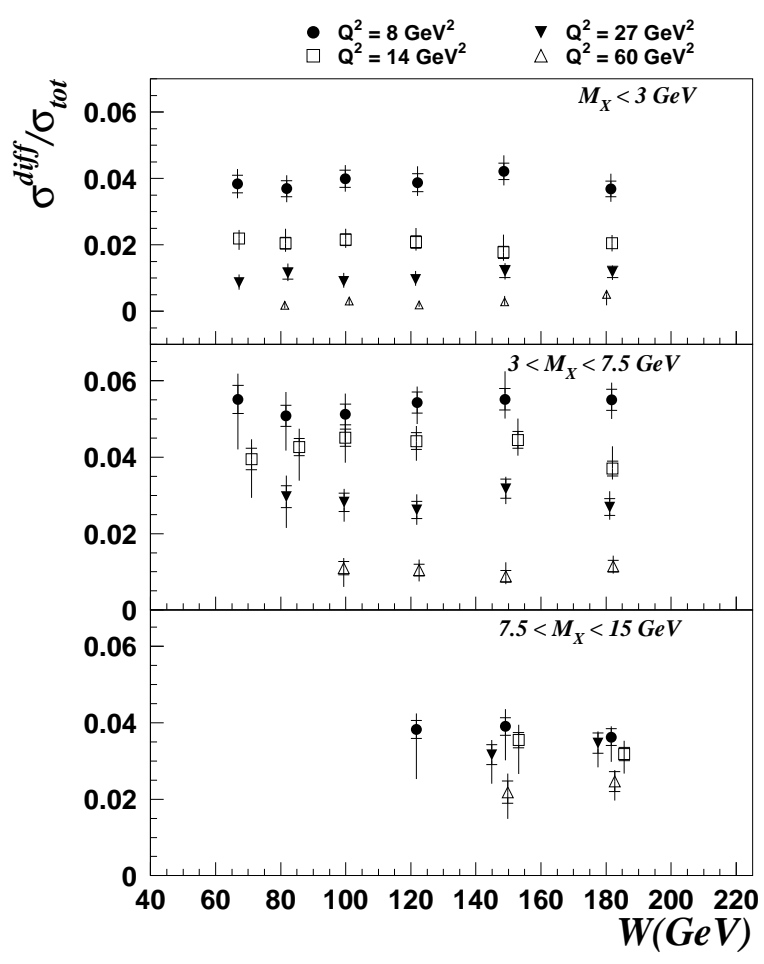

FIG. 56 The ratio of diffractive to inclusive cross sections as a function of $W$, integrated over different $M_{X}$ regions at fixed $Q^{2}$. Obtained using ZEUS 1994 data. From (Breitweg et al., 1999e).

the inclusive cross section. ${ }^{3}$ This is indicative of a large role of gluons in the diffractive exchange Ahmed et al., 1995a). This qualitative conclusion is formalised through the extraction of DPDFs, as described in more detail in this section.

The DPDFs in Eq. 14 represent probability distributions for partons $i$ in the proton under the constraint that the proton is scattered to a particular system $Y$ with a specified four-momentum. They are essentially equivalent to the fracture functions developed in an earlier approach (Trentadue and Veneziano, 1994). They are not known from first principles, but can be determined from fits to the data using the DGLAP evolution equations (Blumlein and Robaschik, 2001). Due to kinematic constraints, it is not possible to access the full range of $\beta$ and $Q^{2}$ using data from only one value of $x_{\mathbb{P}}$ at HERA. The parametrisations of the $x_{\mathrm{IP}}$ dependence of the DPDFs described in Section V.C.4 together with the proton vertex factorisation assumption introduced in Section V.C.3 is therefore adopted, such that data from

\footnotetext{
${ }^{3}$ See (Aktas et al. , 2006e for an alternative comparison between scaling violations in diffractive and inclusive DIS considered at the same $x$ values in each case.
}

multiple $x_{\mathbb{P}}$ values can be used simultaneously in extracting DPDFs which then depend on $\beta$ and $Q^{2}$ only.

DPDFs have been extracted using standard NLO QCD procedures, similar to those employed to extract inclusive proton PDFs, but with smaller numbers of free parameters for the momentum fraction dependence of the parton densities at the starting scale for evolution. This momentum fraction is usually denoted $z$ or $z_{\mathbb{P}}$ (it is equal to $\beta$ when used to describe the quark coupling to the exchange boson). In the fits made to date, only two distinct parton densities are considered: a gluon density and a singlet quark density, which is assumed to be flavour symmetric between up, down and strange quarks and their antiquarks. This latter assumption, particularly on the size of the strange quark density, is rather ad hoc and remains to be tested in detail.

The most recent results obtained when fitting inclusive $\sigma_{r}^{D}$ data alone can be found in (Aktas et al., 2006e; Chekanov et al., 2010a), see Fig. 57. Since $\sigma_{r}^{D}$ measures essentially the charge-squared-weighted sum of quarks, these fits result in tight constraints (to around 5\%) on the singlet quark density. From the scaling violations, the gluon distribution is also rather well constrained at moderate $z$ values (to around $10-15 \%$ ). However, the sensitivity to the gluon density with $z \gtrsim 0.5$ is poor, due to the inevitable dominance of the evolution by the $q \rightarrow q g$ splitting as $z \rightarrow 1$. If diffractive jet production (see Section V.C.6) is used as an additional constraint, the high $z$ gluon precision is improved considerably, at the expense of additional theoretical assumptions on the large $\beta$ dynamics. Results of fits of this type can be found in (Aktas et al., 2007b; Chekanov et al., 2010a), see Fig. 58,

In all fits performed, the DPDFs are dominated by the gluon density, which extends to large values of $z$ and accounts for typically $60 \%$ (ZEUS (Chekanov et al., 2010a)) to 70\% (H1 (Aktas et al., 2006e)) of the total longitudinal momentum of the diffractive exchange.

It is interesting to note that the DPDFs, particularly the quark distribution, resemble the parton densities of the photon (Nisius, 2000). The hadronic structure of the photon is generated from an initial $\gamma \rightarrow q \bar{q}$ splitting, with lower $x$ structure emerging from further splittings and evolution. This superficial similarity is as might be expected if diffraction emerges from a single gluon exchange, dressed in a manner which neutralises the colour. The structure then develops from $g \rightarrow q \bar{q}$ similarly to the photon case, but with an admixture of $g \rightarrow g g$ initial splittings.

More sophisticated approaches to DPDFs exist, notably in which a direct, hard, pomeron contribution is also included (Martin et al., 2007) or which include a higher twist contribution at large $\beta$ (see Section V.C.8) (Golec-Biernat and Luszczak, 2007; Rovon et al., 2001). Whilst these contributions arise naturally in QCD and must be present at some level, the evidence both from these fits and from hadronic final state comparisons (Section V.C.6) is that they are numerically small, compared with the standard resolved structure contribution. The 


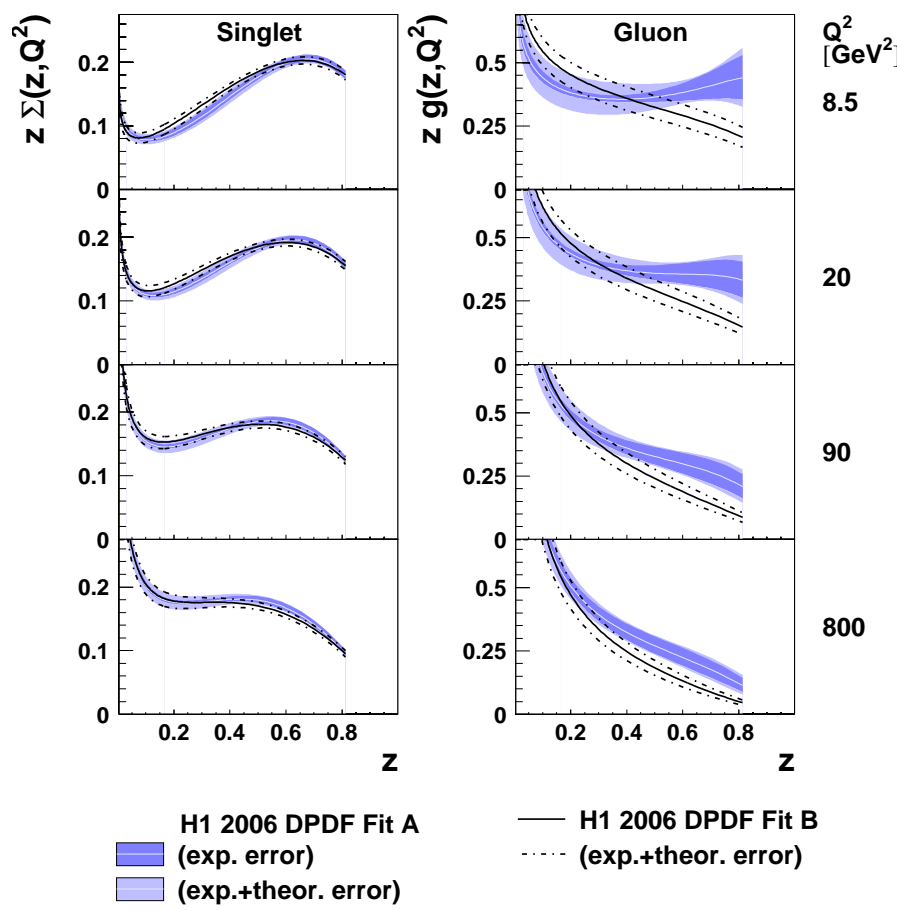

FIG. 57 DPDFs extracted from an H1 fit to inclusive LRG data only. The two different sets of DPDFs shown (Fit A and Fit B) have very similar fit qualities, illustrating the lack of sensitivity to the gluon density at large momentum fractions. From (Aktas et al., 2006e).
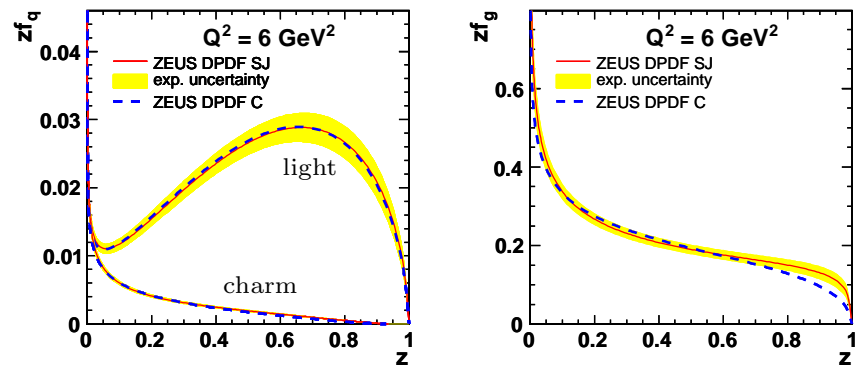

FIG. 58 Diffractive quark (left) and gluon (right) distributions extracted from a ZEUS fit to inclusive LRG and LPS data, with diffractive dijet data also included. From (Chekanov et al., 2010a).

DPDF approach does, however, appear to undergo an infrared breakdown at larger $Q^{2}$ scales than is the case in inclusive QCD fits (Aktas et al., 2006e; Chekanov et al., 2010a). Whilst this may provide further evidence for saturation effects in DDIS (Frankfurt et al., 2001a), it may also be a consequence of enhanced higher twist contributions or a less quickly convergent QCD order expansion in the diffractive than the inclusive case.

\section{Applications of Diffractive Parton Densities}

The DPDFs described in Section V.C.5 and their predecessors have been used to predict a wide variety of observables in DIS and photoproduction at HERA, as well as at the Tevatron and the LHC. At one level, this is done through implementations (usually of the H1 Fit B DPDFs (Aktas et al., 2006e)) in Monte Carlo generators (Cox and Forshaw, 2002; Jung, 1995; Navin, 2010). In particular, the RAPGAP model has been used extensively as an experimental tool for modelling both inclusive and hadronic final state diffractive data at HERA. The DPDFs have also been interfaced to various NLO QCD calculations in order to compare their predictions with measurements.

There is a wealth of literature on this topic, which is summarised with a few examples below. These tests are very similar in design and scope to those discussed in Section [II] However, the level of precision is poorer in the diffractive case, due to smaller data samples, added experimental complications and kinematic restrictions placed for example on jet transverse momenta. ${ }^{4}$

Early measurements of inclusive final state observables in DDIS were sufficient to rule out a diffractive exchange with a quark-dominated structure, by showing that the basic event topology is consistent with the boson-gluon fusion process, $\gamma^{*} g \rightarrow q \bar{q}$ (Fig. [52(b)), yielding copious high $p_{T}$ particle production and leaving behind a colour-octet, single gluon-like, remnant of the diffractive exchange. The full list of inclusive final state observables measured in DDIS now comprises charged particle spectra, multiplicities and their rapidity correlations (Adloff et al., 1998a.b; Chekanov et al., 2002f), energy flows (Adloff et al., 1998a; Chekanov et al., 2002f) and event shapes (thrust and sphericity) (Adloff et al., 1998d; Breitweg et al., 1998d; Chekanov et al., 2002f). These measurements are universally in agreement with predictions based on DPDFs extracted from $\sigma_{r}^{D}$ data, reinforcing the picture of a diffractive exchange dominated by gluons extending to large momentum fractions.

The most precise tests of DPDFs in diffractive DIS have been obtained from exclusive final state observables formed from relatively high transverse momentum jet production, the rate for which is closely related to the diffractive gluon density. Dijet measurements have been made as a function of many different variables (Aaron et al., 2012e; Aktas et al., 2007b; f; Chekanov et al., 2007c) and have been extensively used in comparisons with DPDF-based NLO predictions (Aktas et al., 2007b; Chekanov et al., 2010a). An example is shown in Fig. 59. This sort of comparison has again been universally successful. In fact, the factorisable ('resolved pomeron') model in diffractive DIS works in a wider range of contexts than might be expected. For example, DPDF-based predictions also describe 3jet diffractive final states fairly well (Adloff et al., 2001a;

\footnotetext{
4 The total available invariant mass $M_{X}$ for final state particle production in diffraction is typically a factor of 10 smaller than the analogous variable, $W$, in the inclusive case.
} 
Chekanov et al., 2001c), despite being at the LO, rather than the NLO, level in the 3-jet case. A further intriguing example is the case where one of the reconstructed jets is close in rapidity to the edge of the rapidity gap (Aaron et al., 2012e), a topology which can be measured by exploiting the proton tagging method. This latter case rules out a dominant contribution from hard (pomeron remnant-free) diffractive production of the type discussed in (Hebecker and Teubner, 2001; Martin et al., 2007), which ought not to be describable using the DPDF approach. The strong experimental evidence for proton vertex factorisation and the applicability of DPDFs to diffractive dijets in DIS, together with the relatively high precision with which the momentum fraction $z$ can be reconstructed from the kinematics of the diffractive final state and the jet pair, have led to the argument more recently being reversed, with jet data being used as an input to DPDF extractions (Aktas et al., 2007b; Chekanov et al., 2010a), as discussed in Section V.C.5.
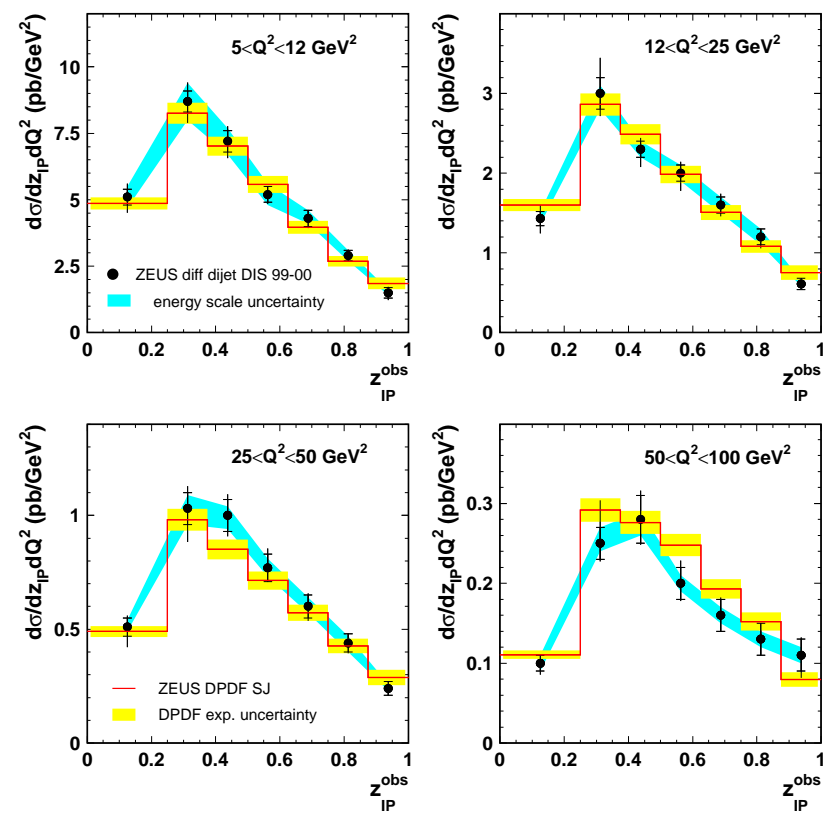

FIG. 59 Comparison of diffractive DIS dijet cross sections, measured double differentially in $z_{\mathbb{P}}$ and $Q^{2}$, with NLO QCD predictions based on DPDFs. In this case, the data shown were used together with inclusive diffraction measurements in the fit, to improve the high $\beta$ sensitivity. From (Chekanov et al., 2010a).

Diffractive open charm production in DIS (Aktas et al., 2007a; Chekanov et al., 2003d) provides a further exclusive final state with high sensitivity to the diffractive gluon density via the lowest order contributing process, $\gamma^{*} g \rightarrow c \bar{c}$. In all measurements made to date, the charm quarks are tagged through the reconstruction of a $D^{*}$ meson using the usual $D^{*}-D^{0}$ mass difference technique (Section [I.D). Due to larger backgrounds and smaller statistics, charm observables have not yielded the precision tests of diffractive factorisation and DPDFs that have been achieved with jets. In principle, due to the weaker kinematic constraints on $M_{X}$, it should be possible to probe smaller $z$ values by using charm as a tag. However, the problems of forward-going track reconstruction experienced by both collaborations has limited progress in this direction. Nonetheless, as can be seen from the example comparisons in Fig. 60, open charm production is well described by DPDF-based models, providing complementary support for this approach and a constraint on the heavy flavour treatment in diffractive QCD fits.

A final class of DPDF tests in DIS may be performed using only inclusive diffractive cross sections. Whilst these tests require measurements at the extremes of the accessible kinematic range, resulting in only limited precision, they are complementary to hadronic final state constraints. An H1 measurement of the diffractive charged current cross section is shown to be consistent with DPDF-based predictions in (Aktas et al., 2006e). This is the only comparison made to date which is sensitive to the light quark flavour decomposition of the diffractive quark density. The rather ad hoc flavourdemocratic assumptions made in the standard QCD fitting procedures (Section V.C.5) are consistent with the data within the large statistical uncertainties.

The diffractive longitudinal structure function, $F_{L}^{D}$ (Newman, 2005) is a further independent observable with sensitivity to both the quark and the gluon densities in novel ways. In particular, it provides a unique test of the low- $x$ gluon. $F_{L}^{D}$ can in principle be measured from variations in the DDIS cross section with the azimuthal angle between the lepton and jet scattering planes, though attempts to observe this effect using the FPS/LPS detectors have yielded results consistent with zero (Chekanov et al., 2009b). Significantly non-zero results have, however, been obtained (Aaron et al., 2012g) using the Rosenbluth technique, comparing diffractive reduced cross section data at fixed $x_{\mathbb{P}}, \beta$ and $Q^{2}$, but different $e p$ centre-of-mass energy, exploiting the data taken at $\sqrt{s}=460 \mathrm{GeV}$ and $\sqrt{s}=525 \mathrm{GeV}$ at the very end of the HERA running. The sensitivity to $F_{L}^{D}$ requires highly challenging measurements at large $y$ (small scattered electron energy). Nonetheless, this structure function has been measured over a fairly large kinematic region. As summarised in Fig. 61, the data are again supportive of the DPDF approach.

\section{Limitations of Diffractive Parton Densities}

Whilst models based on proton vertex factorisation and DPDFs work well to describe all diffractive processes beyond the lowest $M_{X}$ resonance region in DIS, they fail spectacularly when DPDFs extracted from HERA $\sigma_{r}^{D}$ data are applied to diffractive $p \bar{p}$ scattering at the Tevatron. For example, predictions for diffractive dijet production at the Tevatron exceed the data by a factor 

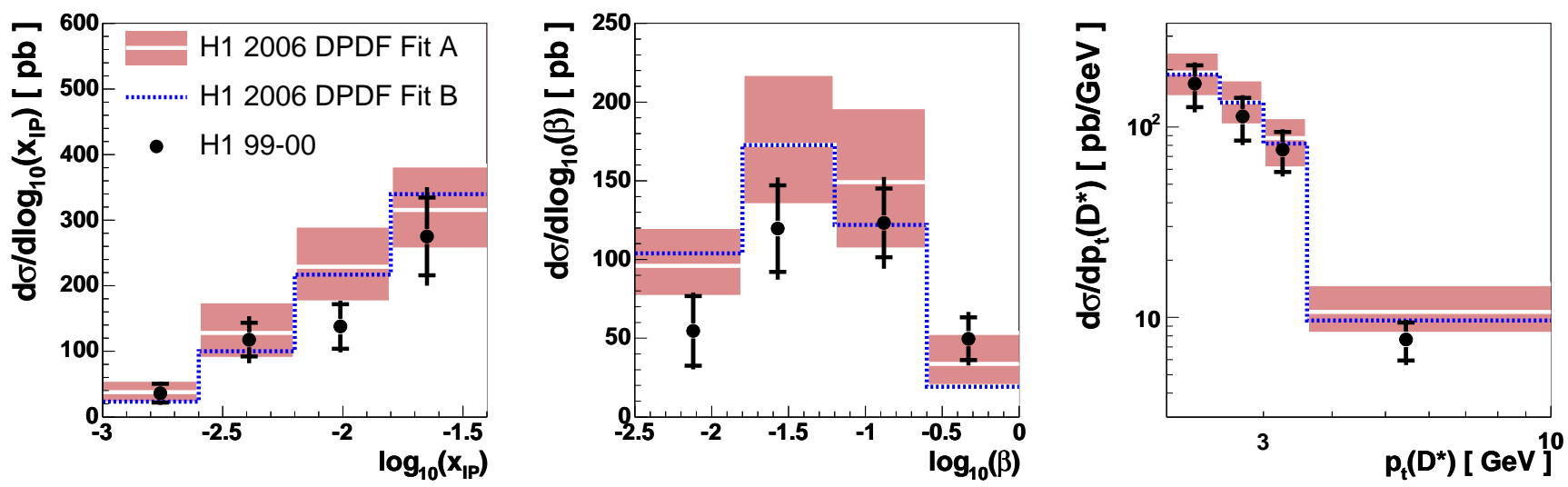

FIG. 60 Comparisons between diffractive open charm $\left(D^{*}\right)$ production measurements and NLO QCD predictions based on DPDFs. From (Aktas et al., 2007a).

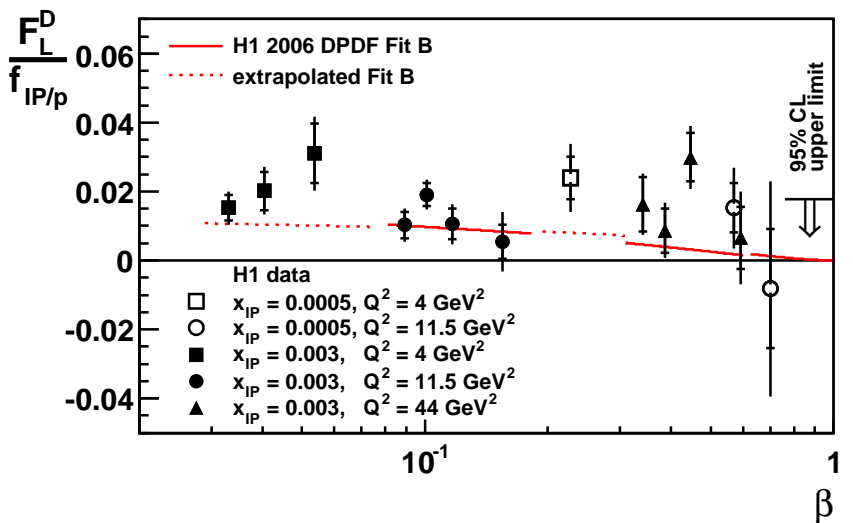

FIG. 61 Summary of H1 measurements of the diffractive longitudinal structure function $F_{L}^{D}$, exploiting data taken with variations in the proton beam energy. To allow comparisons between measurements at different $x_{\mathrm{IP}}$ values, the data are normalised by a diffractive flux factor $f_{\mathbb{P} / p}$ (see Section V.C.4 . The data are compared with an NLO QCD prediction based on DPDFs. From (Aaron et al., 2012g).

of around 10 (Affolder et al., 2000; Klasen and Kramer, 2009). This limitation is predicted as part of the QCD hard scattering factorisation theorem for diffraction (Collins, 1998) and is usually interpreted in terms of multiple scattering, or 'absorptive' effects, which occur in the presence of beam remnants. These effects can be parametrised in terms of a 'rapidity gap survival probability' (Bjorken, 1993; Dokshitzer et al., 1992). Similar effects are emerging at the LHC (Chatrchyan et al., 2013a).

Measurements of diffractive dijet photoproduction have been pursued as a control experiment for gap destruction models. In a lowest order interpretation, direct photon processes ought to be unaffected by such effects, whereas they should be present in resolved pho- ton processes, where the photon interacts through its hadronic structure (Fig. [52(c)). For example, a theoretical model which successfully describes the Tevatron result predicted a suppression of the resolved contribution by a factor of 0.34 (Kaidalov et al., 2003). Multiple measurements of diffractive dijet photoproduction have been made by $\mathrm{H} 1$ and ZEUS, and have been compared with NLO calculations based on DPDFs extracted in DDIS. Whilst the experimental data are just about compatible between the two collaborations, the conclusions from $\mathrm{H} 1$ and ZEUS differ slightly in this area. Notably, neither collaboration sees any evidence for the expected $x_{\gamma}$ dependence of the survival probability. H1 data (Aaron et al., 2010a; Aktas et al., 2007f) suggest a suppression of the data by a factor of around 0.6 relative to NLO QCD predictions, independently of $x_{\gamma}$. ZEUS results, which correspond to larger jet transverse energies (Chekanov et al., 2008c, 2010a) suggest a smaller suppression and are, in fact, consistent with no suppression whatsoever. Selected data from both collaborations are shown in Fig. 62. These apparent problems are at least partially resolved by a recent model in which a more careful treatment of point-like, as distinct from fully resolved, photon structure is introduced (Kaidalov et al., 2010), leading to a much larger, and $E_{T}$ dependent, survival probability for $0.1 \lesssim x_{\gamma}<1$.

Diffractive charm production has also been studied in photoproduction (Aktas et al., 2007a; Chekanov et al., $2007 \mathrm{~b})$. The measurements are kinematically restricted to the large $x_{\gamma}$ direct photon region and are described, within large experimental and theoretical (scale) uncertainties, by DPDF-based predictions without recourse to gap destruction effects.

Rapidity gap survival probabilities and their kinematic dependences remain an area which is not yet fully resolved. This is unfortunate, since in addition to the interest this topic generates in its own right, it is an essential ingredient in predicting hard diffractive cross sections at the LHC. 

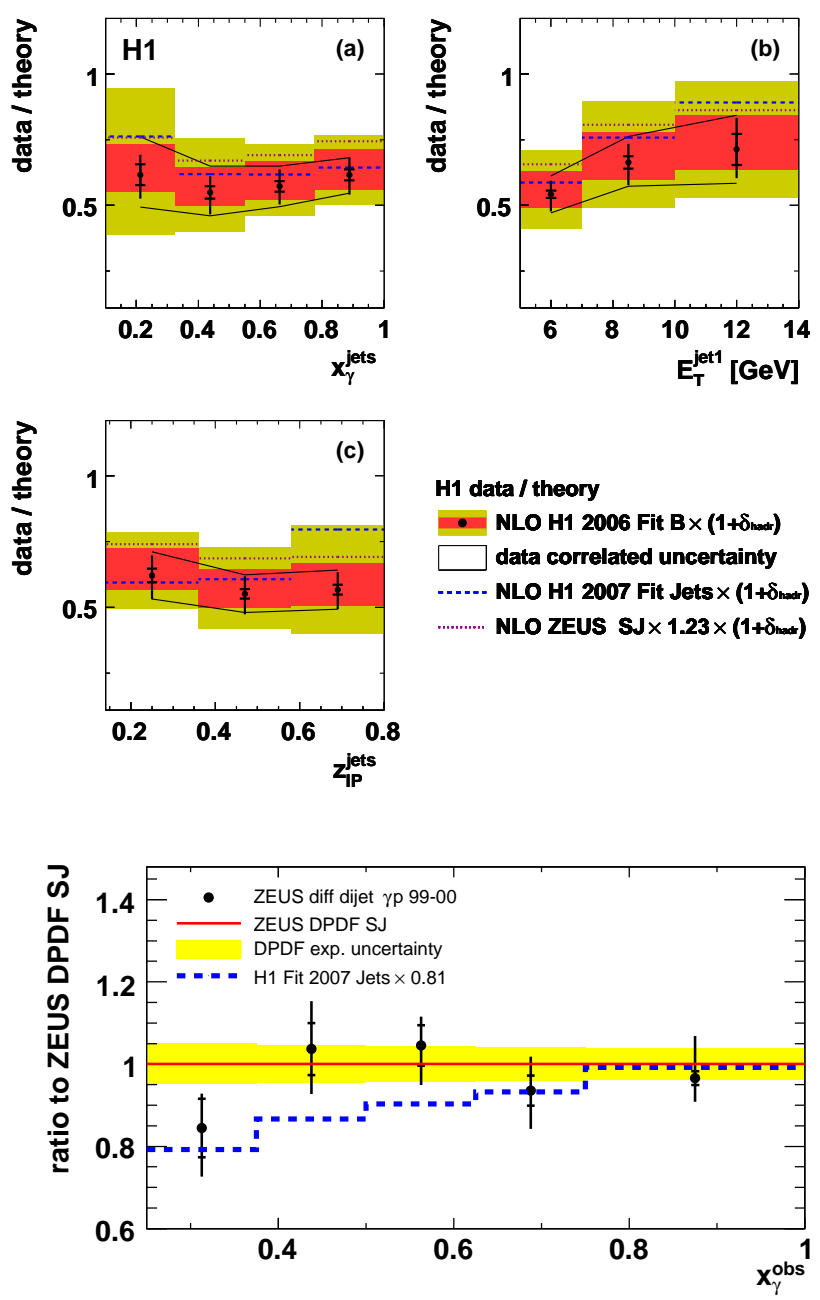

FIG. 62 Diffractive dijet photoproduction data shown as ratios of measured cross sections to DPDF-based NLO predictions (i.e. as measurements of the rapidity gap survival probability). Top: H1 data, from (Aaron et al., 2010a) Bottom: ZEUS data, from (Chekanov et al., 2010a).

\section{Diffractive DIS in Dipole Models}

The application of the dipole picture to inclusive DDIS has proved to be problematic, mainly due to the need for higher multiplicity fluctuations ( $q \bar{q} g$ and perhaps others) in order to describe the large $M_{X}$, small $\beta$ region. This need was first shown in a quantified manner through the 'BEKW' fits to $F_{2}^{D}$ data in (Bartels et al., 1999a). In this parametrisation, the data at low and moderate $\beta$ are described in terms of $q \bar{q}$ and $q \bar{q} g$ dipole fluctuations of transversely polarised photons, whilst the high $\beta$ region contains a $Q^{2}$-suppressed non-leading twist contribution from $q \bar{q}$ fluctuations of longitudinally polarised photons. This approach was further developed for comparisons with inclusive DDIS data for example in the saturation model (Golec-Biernat and Luszczak, 2007; Golec-Biernat and Wusthoff, 1999). An example decom- position of the $\beta$ dependence of DDIS data is shown in Fig. 63. Predictions have also been made for hadronic final state observables, based on a two-gluon exchange model of exclusive $q \bar{q}$ (Bartels et al., 1996b; Lotter, 1997) and $q \bar{q} g$ (Bartels et al., 2002, 1999b) production. However, progress has been limited by significant theoretical uncertainties associated with the low $\beta q \bar{q} g$ contribution and a lack of direct evidence for the high $\beta$ higher twist $q \bar{q}$ term.

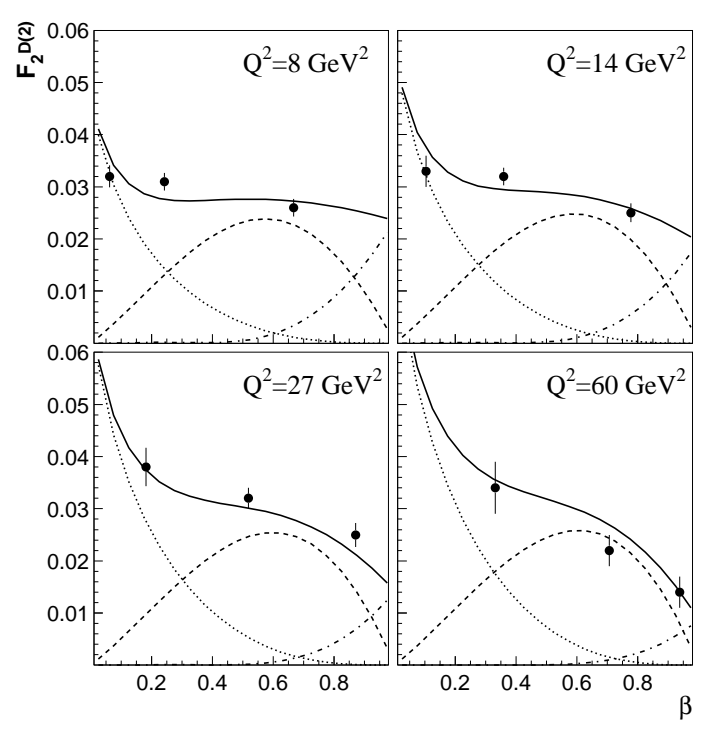

FIG. 63 An example decomposition of the $\beta$ dependence of ZEUS DDIS data (Breitweg et al., 1999e) into different dipole terms, according to the parametrisation in (Golec-Biernat and Wusthoff, 1999). The dashed, dotted and dot-dashed curves correspond to leading twist transverse $q \bar{q}$, leading twist transverse $q \bar{q} g$ and higher twist longitudinal $q \bar{q}$ contributions, respectively.

\section{Leading Protons and Neutrons Beyond the Pomeron Region}

Both H1 and ZEUS had proton spectrometers (see Fig. (7) with acceptance in the range $0.1 \lesssim x_{\mathrm{IP}} \lesssim 0.3$, well beyond the classic diffractive region where pomeron exchange is expected to dominate. In this region, the $e p \rightarrow e X p$ cross section may be understood in terms of the exchange of 'sub-leading' exchanges of neutral meson states (Adloff et al., 1999d; Chekanov et al., 2009c). In the case of ZEUS, the scattered proton sensitivity extended as far as $x_{\mathrm{IP}} \sim 0.7$. The acceptance of the zero degree forward neutron calorimeters in both experiments extended over a very wide range in neutron energies, from low values, where neutron production is describable by standard proton fragmentation, to large values, where charged colour singlet exchange $e p \rightarrow e X n$ becomes dominant. Interpreted in terms of meson tra- 
jectory exchanges, this latter reaction proceeds only via isospin-1 exchanges, for which the relative rates of leading neutron and leading proton production are simply related via a Clebsch-Gordan coefficient of 2. Studying the leading proton and leading neutron data together in a Regge pole model (e.g. (Szczurek et al., 1998)) leads to the tentative conclusion that the sub-leading trajectory with $\alpha_{\mathbb{R}}(0) \sim 0.5$ in Eq. 16, which becomes important for $x_{\mathbb{P}} \simeq 0.05$, may be that associated with the $f_{2}$ meson.

At larger $x_{\mathrm{IP}}$ values, leading proton and neutron production can only be described simultaneously if the exchanged trajectory is dominantly the pion, with $\alpha_{\pi}(0) \simeq$ 0 (Bishari, 1972), though other contributions also appear to be present (Kaidalov et al., 2006; Khoze et al., 2006). The study of leading neutron data at relatively large neutron energies therefore raises the interesting, but not uncontroversial (Frankfurt et al., 1997), opportunity of measuring the partonic structure of the pion using a similar factorisation between pion flux and structure function to that described for the pomeron in Section V.C.3.

For $x_{\mathrm{IP}} \lesssim 0.3$, reconstructed using the neutron energy $E_{n}$ and assuming exclusive production at the proton vertex via $x_{\mathrm{IP}} \equiv 1-E_{n} / E_{p}$, inclusive leading neutron data in DIS (Aaron et al., 2010f; Chekanov et al., 2005h, 2007i) are broadly consistent with the pion exchange hypothesis. An example analysis is shown in Fig. 64. Leading neutron data at $x_{\mathbb{I P}}=0.27$ are shown after dividing by a parametrisation of the pion flux. Up to residual contributions from standard fragmentation processes and possibly other isovector exchanges, the data then correspond to the pion structure function, $F_{2}^{\pi}\left(\beta, Q^{2}\right)$. Existing pion structure function parametrisations are broadly in-line with the data, but clearly overshoot when considered in detail. A simple model based on valence quark counting, such that $F_{2}^{\pi}=2 F_{2} / 3$, is slightly closer to the data. Considering the large uncertainties in the pion flux factor and the lack of previous data sensitive to the measured range, which extends to $\beta<10^{-3}$, the level of agreement is reasonable. An analysis in which HERA leading neutron data are used as an input to a pion parton density extraction is, however, yet to be performed.

In addition to the inclusive neutron production process, measurements have also been made of dijet (Aktas et al., 2005d; Chekanov et al., 2010c) and charm quark (Chekanov et al., 2004g) photoproduction in association with leading neutrons. Considered together with the inclusive leading neutron data, these final state measurements show evidence for considerable absorptive corrections. This complication, together with the uncertainties inherent in factoring out the pion flux, have limited the information finally extracted on the pion structure function.

\section{E. Very Hard Diffraction and the BFKL Pomeron}

For the diffractive processes discussed in the previous sections, $|t|$ is generally smaller than typical hadronic

$$
F_{2}^{\mathrm{LN}(3)}\left(x_{\mathrm{L}}=0.73\right) / \Gamma_{\pi}, \Gamma_{\pi}=0.13
$$
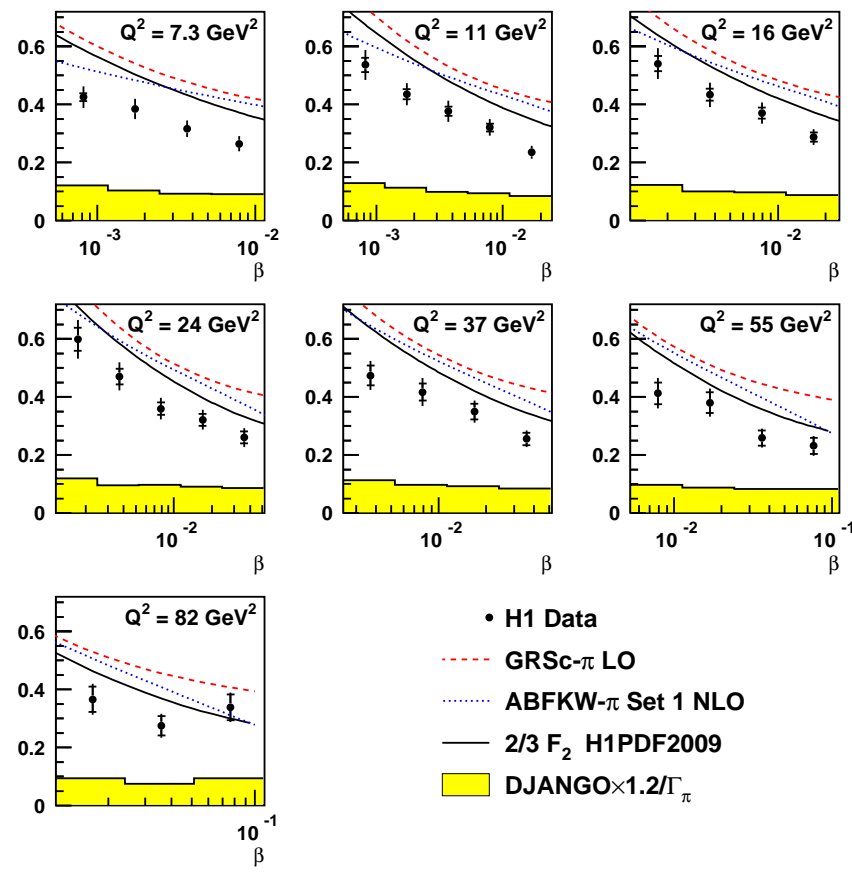

FIG. 64 Leading neutron structure function data (integrated over neutron transverse momenta up to $200 \mathrm{MeV}$ ), divided by a parametrisation (Holtmann et al., 1994) of the pion flux $\Gamma_{\pi}$ at $x_{L} \equiv 1-x_{\mathbb{P}}=0.73$. The estimated contribution from standard fragmentation processes is shown as a yellow band. After accounting for this, the data are compared with two parametrisations of the pion structure function (Aurenche et al., 1989; Gluck et al., 1999) and with a parametrisation of the proton structure function (Aaron et al., 2009b) multiplied by 2/3. From (Aaron et al. 2010f).

mass scales. However, diffractive processes have also been studied at HERA at $|t|$ values which are large enough to provide a hard scale in their own right. Under such circumstances, the $t$ channel pomeron exchange ought to be genuinely hard in the sense that it couples as a whole to individual partons. Cases where the hard subprocess satisfies $\hat{s} \gg-\hat{t} \gg \Lambda_{\mathrm{QCD}}$ correspond to a perturbatively calculable limit of Regge theory. Where there is no particular ordering in transverse momentum within the colour singlet exchange, the dynamics may be driven primarily by BFKL evolution (see Section III.D). Diffractive processes at large $|t|$ therefore represent a promising area in which to search for low $x$ parton dynamics driven by the BFKL pomeron.

One striking signature for perturbative colour singlet exchange is the photoproduction of pairs of jets separated by a large rapidity gap. For such 'gap between jets' configurations, $|t| \approx p_{\mathrm{T} \text {, jet }}^{2}$ is very large, typically $|t|>25 \mathrm{GeV}^{2}$ in HERA studies, and calculations using the leading-logarithmic BFKL approxima- 
tion (Mueller and Tang, 1992) with appropriate modifications (Enberg et al., 2002a; Forshaw and Sutton, 1998) can be applied to the scattering between a parton from the proton and a parton from the resolved structure of the photon. However, the situation is complicated by the possibility of secondary scattering and a rapidity gap survival probability significantly smaller than unity, similar to that discussed for the diffractive dijet photoproduction process in Section V.C.7. The HERA data (Adloff et al., 2002b; Chekanov et al., 2007n; Derrick et al., 1996b) have shown clear evidence for events with little energy flow between jets, which occur more frequently than expected from hadronisation fluctuations in standard Monte Carlo models of jet photoproduction. The order of magnitude of the signal is also in agreement with that expected from the BFKL calculations. Whilst this is highly suggestive, and is in fact perhaps the best evidence for BFKL dynamics at HERA, more quantitative conclusions have been precluded by the uncertainties in the perturbative calculations, the gap survival probability and the residual non-diffractive contributions.

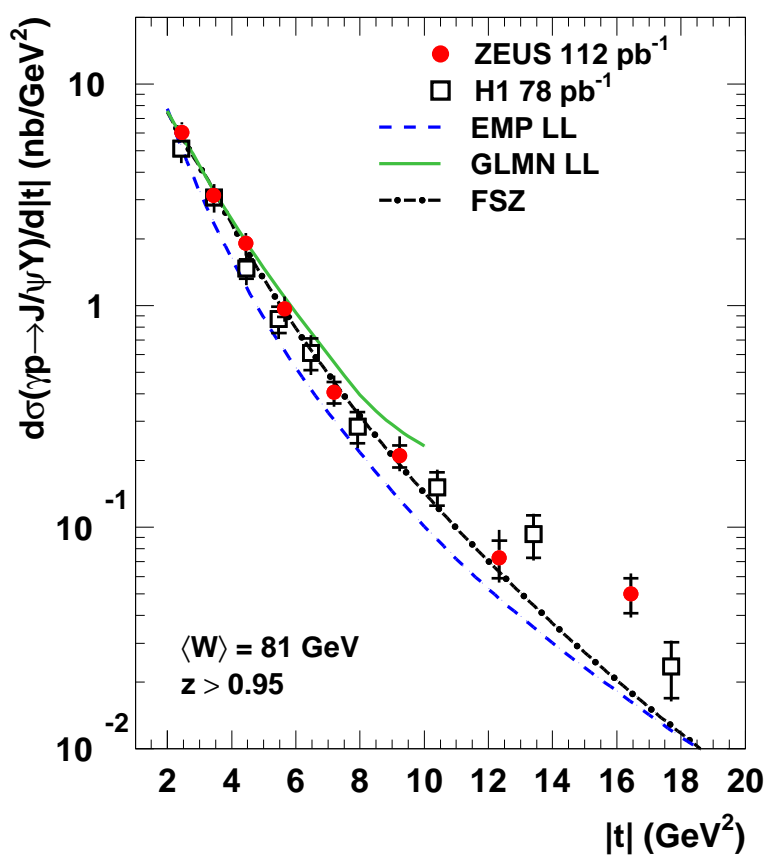

FIG. 65 Dependence on $|t|$ of proton dissociative $J / \psi$ production integrated over proton dissociation masses satisfying $M_{Y}^{2} / W^{2}<0.05$. The data are compared with a prediction ('GLMN LL' (Gotsman et al., 2002)) which is based on DGLAP evolution and is expected to be valid for $|t|<m_{\psi}^{2}$. They are also compared with a BFKL-based prediction ('EMP LL' (Enberg et al., 2002b)) and a more phenomenological approach ('FSZ' (Frankfurt et al., 2008). From (Chekanov et al., 2010f).

Another candidate process in which to observe perturbative colour singlet exchange is quasi-elastic vec- tor meson production at large $|t|$. Although $|t|$ values are smaller here than in the gaps-between-jets case and the proton essentially always dissociates, the exclusive production mechanism at the photon vertex (Fig. 45(b)) implies significantly reduced complications from gap destruction effects, even in photoproduction. The possibility of perturbative colour singlet exchange has long been considered for this process (Ginzburg et al., 1987) and detailed calculations have been performed in the leading-logarithmic BFKL framework (Bartels et al., 1996a; Enberg et al., 2003; Forshaw and Poludniowski, 2003; Forshaw and Ryskin, 1995; Poludniowski et al., 2003). BFKL evolution is expected to be relevant for $|t|>m_{V}^{2}$, where $m_{V}$ is the vector meson mass. For the case of heavy vector meson production, there is an interim region where $|t|<m_{V}^{2}$, yet $|t|$ remains large enough to apply perturbative techniques. Here, the transverse momenta along the gluon ladder remain ordered and DGLAP dynamics are expected to apply (Blok et al., 2010; Gotsman et al., 2002). Studies have been made at HERA for light vector mesons (Aktas et al., 2006b; Chekanov et al., 2003b) and $J / \psi$ (Aktas et al., 2003; Chekanov et al., 2010f) mesons, as well as of exclusively produced photons (Aaron et al., 2009d). Since it offers adequate statistics as well as a relatively clean theoretical interpretation, the $J / \psi$ channel has yielded the most precise tests. An example analysis is shown in Fig. 65. The $t$ dependences generally follow the expected approximate power law behaviour at large $|t|$ and the effective trajectory slope $\alpha_{\mathbb{P}}^{\prime}$ is much smaller than that describing soft diffraction, as expected in a BFKL treatment. However, the overall description by specific BFKL predictions is not yet sufficiently good for strong claims to be made.

\section{SUMMARY AND OUTLOOK}

Condensing into the previous 50 pages a total of about 200 publications from H1 and ZEUS on the hadronic final state has been a challenge. Further reducing this to a 1-page summary is a task fraught with omissions and generalisations. With this caveat, the following represents an attempt to summarise the essential highlights of studies of the HERA hadronic final state as briefly as possible.

- Measurements of jet production among the most precise in the world, which have led to significant constraints on the parton density functions in the proton and precise determinations of $\alpha_{s}$.

- Similarly, data on heavy-quark production which have led to important constraints on the charm contribution to the proton structure functions, unique to HERA, and on the heavy quark masses.

- A demonstration of the power of QCD and in particular the applicability of the DGLAP equations 
for a multitude of processes covering a wide kinematic range. Despite considerable attempts to isolate evidence for BFKL or other parton evolution schemes and a handful of measurements which have yet to be satisfactorily described, no unequivocal evidence has been found for the need for nonDGLAP dynamics.

- Many measurements of light and heavy quark fragmentation processes and charged particle spectra which show similar behaviour to that observed in $e^{+} e^{-}$or other collisions. Despite some anomalies, the data are broadly consistent with the concept of universal fragmentation.

- A wide variety of searches for evidence of exotic QCD processes, such as pentaquarks and instantons. Of all searches performed, a glueball candidate decaying to a $K_{S}^{0} K_{S}^{0}$ pair with a mass consistent with the $f_{0}(1710)$ state, was the only clear signal.

- A detailed exploration of the quasi-elastic exclusive production of vector mesons $\left(\gamma^{(*)} p \rightarrow V p\right)$ and photons $\left(\gamma^{(*)} p \rightarrow \gamma p\right)$ over a wide range in scale, an appropriate choice for which is often $\left(Q^{2}+M_{V}^{2}\right) / 4$. The data beautifully illustrate and explore the transition with scale from a regime familiar from soft hadronic elastic and diffractive scattering to a region governed by hard diffractive exchanges which can be interpreted in terms of partons.

- Precise measurements of hard exclusive diffractive processes, for example $J / \psi$ photoproduction, and the development of successful methods for calculating related observables starting from a knowledge of the parton densities of the proton.

- The precise measurement and interpretation of inclusive diffraction over a vast, usually threedimensional, kinematic range, which has shown the process to be well modelled as the deep inelastic scattering of the electron from a factorisable soft object, not dissimilar from the 'pomeron' of soft hadronic physics.

- The extraction of the partonic structure of the softpomeron-like exchange and its successful application in predicting diffractive final state observables using standard NLO DGLAP-based tools.

Although these essential points are now unlikely to alter, the $\mathrm{H} 1$ and ZEUS collaborations are still regularly publishing new results at the time of writing, and in several important areas the most precise measurements are still yet to come. Examples which have been discussed here include inclusive jet production in DIS and dijet photoproduction, for both of which only around $10 \%$ of the available data has been used in published measurements. Extending to the full dataset and, where possible, combining $\mathrm{H} 1$ and ZEUS data will improve the precision and accessible kinematic range at high $E_{T}$ (equivalently large $x$ ). The precision on heavy flavour cross sections, particularly those in the beauty sector, is also often statistically limited at present, with substantial power to improve if the full HERA data are exploited. In many areas, the understanding of the existing data can be improved considerably by the application of theoretical or phenomenological techniques which in principle already exist. Most prominently, the development for $e p$ scattering of a Monte Carlo model which matches NLO QCD calculations to parton showers would represent a major breakthrough in our ability to model and interpret jet and other final state data. A major impact could also be achieved by feeding into HERA analyses the improved understanding of the underlying event and of hadronisation which is currently developing through model-tuning exercises at the LHC. The impact of these phenomenology improvements would be greatest in allowing low $E_{T}$ data to be exploited more fully, leading to better constraints on photon and low- $x$ proton structure. New parametrisations of photon structure which include a wider range of HERA photoproduction data would also be a significant step forward. Many of the above improvements would extend the list of HERA observables which could reliably be included in fits to extract information on the proton structure. Combining the final inclusive DIS HERA and fixed target data with carefully chosen HERA hadronic final state measurements has the potential to produce constraints on the proton PDFs, $\alpha_{s}$ and heavy quark masses which are unlikely to be surpassed for many years. Indeed, it may not be too ambitious to attempt a simultaneous extraction of proton and photon structure from HERA and other data, a programme of work which may gain new impetus should a high energy $e^{+} e^{-}$linear collider be constructed.

Whilst there is still considerable room for improvement for the topics discussed above, there are also areas of the HERA physics programme which are essentially complete. Given the current experimental precision and the limitations in our theoretical understanding, further substantial progress in areas such as the search for novel low $x$ dynamics, the measurement of charged particle spectra and the unravelling of soft and hard contributions to diffractive DIS appears unlikely. Particularly for unresolved issues in low $x$ physics such as parton saturation, we may have to wait for data from a future higher energy lepton-hadron collider before drawing firm conclusions.

The impact of HERA hadronic final state data and the new techniques developed for its analysis has been felt strongly at the LHC. Apart from the obvious need for a precise knowledge of the proton structure as obtained from the well-matched HERA range of sensitivity, $\mathrm{H} 1$ and ZEUS input has contributed significantly in deciding how to make well-defined measurements of, for example, jet, heavy quark and diffractive processes. There are also less obvious fields which have benefited, for example the modelling of high energy cosmic ray air showers 
and of the hadronic final states produced when neutrinos interact with hadronic matter in neutrino experiments. Whenever and wherever the next facility for high energy lepton-hadron scattering is built, the HERA results will give strong steers as to how and where to make precise measurements of familiar physics and to look for new phenomena, such as low $x$ parton saturation.

In a sentence, the hadronic final state in electronproton collisions at HERA has provided a rich source of data and deepened our understanding of strong interactions.

\section{Acknowledgements}

PRN and MW are fortunate to have been members of the $\mathrm{H} 1$ and ZEUS collaborations, respectively, for around two decades. There are far too many colleagues with whom we have worked closely to name in person, but we wish to record our thanks for many pleasant working relationships and much intellectual stimulation. We would also like to thank J. Butterworth for providing input in the initial planning stages of this review.

\section{References}

Aad, G., et al. (ATLAS), 2011a, Nucl.Phys. B850, 387. Aad, G., et al. (ATLAS), 2011b, Phys.Rev. D83, 112001. Aad, G., et al. (ATLAS), 2011c, Eur.Phys.J. C71, 1636. Aad, G., et al. (ATLAS), 2012a, Phys. Rev. D86, 014022. Aad, G., et al. (ATLAS), 2012b, Eur.Phys.J. C72, 1926. Aad, G., et al. (ATLAS), 2012c, Phys.Rev. D86, 072004. Aaij, R., et al. (LHCb), 2011, Eur.Phys.J. C71, 1645. Aaij, R., et al. (LHCb), 2013, J.Phys. G40, 045001. Aamodt, K., et al. (ALICE), 2010, Phys.Rev. D82, 052001. Aamodt, K., et al. (ALICE), 2011, Phys.Lett. B696, 328. Aaron, F., et al. (H1), 2007, Phys.Lett. B654, 148. Aaron, F., et al. (H1), 2008a, Phys.Lett. B659, 796. Aaron, F., et al. (H1), 2009a, Phys.Lett. B674, 257. Aaron, F., et al. (H1), 2009b, Eur.Phys.J. C64, 561. Aaron, F., et al. (H1), 2009c, Phys.Lett. B681, 391. Aaron, F., et al. (H1), 2009d, Phys.Lett. B672, 219. Aaron, F., et al. (H1), 2009e, Phys.Lett. B681, 125. Aaron, F., et al. (H1), 2009f, Eur.Phys.J. C59, 589. Aaron, F., et al. (H1), 2010a, Eur.Phys.J. C70, 15. Aaron, F., et al. (H1), 2010b, JHEP 1005, 032. Aaron, F., et al. (H1 and ZEUS), 2010c, JHEP 1003, 035. Aaron, F., et al. (H1), 2010d, Eur.Phys.J. C68, 401. Aaron, F., et al. (H1), 2010e, Eur.Phys.J. C67, 1. Aaron, F., et al. (H1), 2010f, Eur.Phys.J. C68, 381. Aaron, F., et al. (H1), 2011a, Eur.Phys.J. C71, 1509. Aaron, F., et al. (H1), 2011b, Eur.Phys.J. C71, 1769, erratum-ibid. 2012, C72, 2252.

Aaron, F., et al. (H1), 2011c, Eur.Phys.J. C71, 1578. Aaron, F., et al. (H1 and ZEUS), 2012a, Eur.Phys.J. C72, 2175.

Aaron, F., et al. (H1), 2012b, Eur.Phys.J. C72, 2074. Aaron, F., et al. (H1), 2012c, Eur.Phys.J. C72, 2047. Aaron, F., et al. (H1), 2012d, Eur.Phys.J. C72, 2148. Aaron, F., et al. (H1), 2012e, Eur.Phys.J. C72, 1970.
Aaron, F., et al. (H1), 2012f, Eur.Phys.J. C72, 1910. Aaron, F., et al. (H1), 2012g, Eur.Phys.J. C72, 1836. Aaron, F. D., et al. (H1), 2008b, Eur.Phys.J. C54, 371. Aaron, F. D., et al. (H1), 2008c, Phys.Lett. B665, 139. Aaron, F. D., et al. (H1), 2008d, Eur.Phys.J. C54, 389. Aaron, F. D., et al. (H1 and ZEUS), 2009g, JHEP 10, 013. Aaron, F. D., et al. (H1), 2010g, Eur.Phys.J. C65, 363. Aaron, F. D., et al. (H1), 2010h, Eur.Phys.J. C67, 1. Aaron, F. D., et al. (H1), 2010i, Eur.Phys.J. C65, 89. Aaron, F. D., et al. (H1), 2010j, Eur.Phys.J. C66, 17. Abachi, S., et al. (D0), 1995a, Phys.Rev.Lett. 74, 3548. Abachi, S., et al. (D0), 1995b, Phys.Lett. B357, 500. Abazov, V. M., et al. (D0), 2003, Phys.Rev. D67, 052001. Abbiendi, G., M. Bonori, R. Brugnera, R. Carlin, V. Chiaratti, et al., 1993, Nucl.Instrum.Meth. A333, 342. Abbiendi, G., et al. (OPAL), 2002, Phys.Lett. B533, 207. Abbott, B., et al. (D0), 2000a, Phys.Rev.Lett. 85, 5068. Abbott, B., et al. (D0), 2000b, Phys.Rev.Lett. 84, 5478. Abbott, B., et al. (D0), 2000c, Phys.Lett. B487, 264. Abdesselam, A., et al., 2011, Eur.Phys.J. C71, 1661. Abe, F., et al. (CDF), 1993a, Phys.Rev.Lett. 70, 713. Abe, F., et al. (CDF), 1993b, Phys.Rev.Lett. 71, 2396. Abe, F., et al. (CDF), 1993c, Phys.Rev.Lett. 71, 500. Abe, F., et al. (CDF), 1993d, Phys.Rev. D47, 4857. Abe, F., et al. (CDF), 1994, Phys.Rev. D50, 5518. Abe, F., et al. (CDF), 1995, Phys.Rev.Lett. 75, 1451. Abe, F., et al. (CDF), 1996, Phys.Rev. D53, 1051. Abe, F., et al. (CDF), 1997a, Phys.Rev. D56, 3811. Abe, F., et al. (CDF), 1997b, Phys.Rev.Lett. 79, 584. Abelev, B., et al. (ALICE), 2012a, Phys.Rev.Lett. 108, 082001.

Abelev, B., et al. (ALICE), 2012b, JHEP 1211, 065.

Abelev, B., et al. (ALICE), 2013, Eur.Phys.J. C 73, 2456.

Abelleira Fernandez, J., et al. (LHeC Study Group), 2012, J.Phys. G39, 075001.

Abramov, V., B. Y. Baldin, A. Buzulutskov, V. Y. Glebov, A. Dyshkant, et al., 1987, Sov.J.Nucl.Phys. 45, 845.

Abramowicz, H., A. Caldwell, and R. Sinkus, 1995, Nucl.Instrum.Meth. A365, 508.

Abramowicz, H., et al. (ZEUS), 2010a, Eur.Phys.J. C70, 965. Abramowicz, H., et al. (ZEUS), 2010b, Phys.Lett. B691, 127. Abramowicz, H., et al. (ZEUS), 2010c, Eur.Phys.J. C69, 347. Abramowicz, H., et al. (ZEUS), 2010d, JHEP 1011, 009. Abramowicz, H., et al. (ZEUS), 2010e, JHEP 1006, 009. Abramowicz, H., et al. (ZEUS), 2011a, Eur.Phys.J. C71, 1573.

Abramowicz, H., et al. (ZEUS), 2011b, Eur.Phys.J. C71, 1659.

Abramowicz, H., et al. (ZEUS), 2012a, Nucl.Phys. B864, 1. Abramowicz, H., et al. (ZEUS), 2012b, Phys.Lett. B715, 88. Abramowicz, H., et al. (ZEUS), 2012c, Phys.Lett. B708, 14. Abramowicz, H., et al. (H1 and ZEUS), 2013a, Eur.Phys.J. C73, 2311.

Abramowicz, H., et al. (ZEUS), 2013b, JHEP 1309, 058. Abramowicz, H., et al. (ZEUS), 2013c, JHEP 1302, 071.

Abramowicz, H., et al. (ZEUS), 2013d, Nucl.Phys. B866, 229. Abramowicz, H., et al. (ZEUS), 2014, Phys.Lett B730, 293. Abreu, P., et al. (DELPHI), 1993, Phys.Lett. B311, 408. Abt, I., et al. (H1), 1994a, Z.Phys. C63, 377.

Abt, I., et al. (H1), 1994b, Phys.Lett. B328, 176.

Abt, I., et al. (H1), 1997a, Nucl.Instrum.Meth. A386, 310. Abt, I., et al. (H1), 1997b, Nucl.Instrum.Meth. A386, 348. Abulencia, A., et al. (CDF), 2007, Phys.Rev.Lett. 99, 132001. Acciarri, M., et al. (L3), 2001, Phys.Lett. B501, 173. 
Acosta, D., et al. (CDF), 2002, Phys.Rev. D65, 052005. Acosta, D., et al. (CDF), 2004, Phys.Rev. D70, 072002. Adams, M., et al. (E665), 1997, Z.Phys. C74, 237. Adler, C., et al. (STAR), 2001, Phys.Rev.Lett. 87, 262301. Adler, S., et al. (PHENIX), 2005, Phys.Rev.Lett. 94, 122302. Adloff, C., et al. (H1), 1997a, Z.Phys. C75, 437. Adloff, C., et al. (H1), 1997b, Z.Phys. C74, 221. Adloff, C., et al. (H1), 1997c, Nucl.Phys. B504, 3. Adloff, C., et al. (H1), 1997d, Z.Phys. C76, 613. Adloff, C., et al. (H1), 1997e, Nucl.Phys. B485, 3. Adloff, C., et al. (H1), 1997f, Phys.Lett. B406, 256. Adloff, C., et al. (H1), 1998a, Phys.Lett. B428, 206. Adloff, C., et al. (H1), 1998b, Eur.Phys.J. C5, 439. Adloff, C., et al. (H1), 1998c, Eur.Phys.J. C5, 575. Adloff, C., et al. (H1), 1998d, Eur.Phys.J. C1, 495. Adloff, C., et al. (H1), 1999a, Eur.Phys.J. C10, 363. Adloff, C., et al. (H1), 1999b, Phys.Lett. B462, 440. Adloff, C., et al. (H1), 1999c, Nucl.Phys. B545, 3. Adloff, C., et al. (H1), 1999d, Eur.Phys.J. C6, 587. Adloff, C., et al. (H1), 1999e, Phys.Lett. B467, 156, erratumibid. 2001, B518, 331.

Adloff, C., et al. (H1), 2000a, Eur.Phys.J. C13, 371. Adloff, C., et al. (H1), 2000b, Eur.Phys.J. C14, 255. Adloff, C., et al. (H1), 2000c, Phys.Lett. B483, 36. Adloff, C., et al. (H1), 2000d, Eur.Phys.J. C12, 595. Adloff, C., et al. (H1), 2001a, Eur.Phys.J. C20, 29. Adloff, C., et al. (H1), 2001b, Eur.Phys.J. C19, 289. Adloff, C., et al. (H1), 2001c, Phys.Lett. B520, 183. Adloff, C., et al. (H1), 2001d, Phys.Lett. B515, 17. Adloff, C., et al. (H1), 2002a, Phys.Lett. B539, 25. Adloff, C., et al. (H1), 2002b, Eur.Phys.J. C24, 517. Adloff, C., et al. (H1), 2002c, Eur.Phys.J. C25, 41. Adloff, C., et al. (H1), 2002d, Eur.Phys.J. C25, 25. Adloff, C., et al. (H1), 2002e, Phys.Lett. B542, 193. Adloff, C., et al. (H1), 2002f, Phys.Lett. B544, 35. Adloff, C., et al. (H1), 2002g, Eur.Phys.J. C25, 495. Adloff, C., et al. (H1), 2003, Eur.Phys.J. C29, 497. Adolph, C., et al. (COMPASS), 2012, Nucl.Phys. B865, 1. Affolder, T., et al. (CDF), 2000, Phys.Rev.Lett. 84, 5043. Ahle, L., et al. (E802), 1998, Phys.Rev. C57, 1416. Ahmed, T., et al. (H1), 1992, Phys.Lett. B297, 205. Ahmed, T., et al. (H1), 1994a, Nucl.Phys. B429, 477. Ahmed, T., et al. (H1), 1994b, Phys.Lett. B338, 507. Ahmed, T., et al. (H1), 1995a, Phys.Lett. B348, 681. Ahmed, T., et al. (H1), 1995b, Nucl.Phys. B435, 3. Aid, S., et al. (H1), 1995, Z.Phys. C69, 27.

Aid, S., et al. (H1), 1996a, Z.Phys. C72, 573.

Aid, S., et al. (H1), 1996b, Nucl.Phys. B472, 3.

Aid, S., et al. (H1), 1996c, Nucl.Phys. B463, 3.

Aid, S., et al. (H1), 1996d, Z.Phys. C70, 17.

Airapetian, A., et al. (HERMES), 2000, Eur.Phys.J. C17, 389.

Airapetian, A., et al. (HERMES), 2007, Phys.Rev. D75, 011103.

Airapetian, A., et al. (HERMES), 2009, Phys.Lett. B679, 100 .

Airapetian, A., et al. (HERMES), 2012a, JHEP 1207, 032. Airapetian, A., et al. (HERMES), 2012b, JHEP 1210, 042. Akers, R., et al. (OPAL), 1994, Z. Phys. C63, 197. Akers, R., et al. (OPAL), 1995, Z.Phys. C67, 203. Aktas, A., et al. (H1), 2003, Phys.Lett. B568, 205. Aktas, A., et al. (H1), 2004a, Phys.Lett. B588, 17. Aktas, A., et al. (H1), 2004b, Eur.Phys.J. C36, 441. Aktas, A., et al. (H1), 2004c, Eur.Phys.J. C33, 477.
Aktas, A., et al. (H1), 2004d, Eur.Phys.J. C36, 413. Aktas, A., et al. (H1), 2004e, Eur.Phys.J. C37, 141. Aktas, A., et al. (H1), 2005a, Eur.Phys.J. C38, 447. Aktas, A., et al. (H1), 2005b, Eur.Phys.J. C41, 453. Aktas, A., et al. (H1), 2005c, Phys.Lett. B621, 56. Aktas, A., et al. (H1), 2005d, Eur.Phys.J. C41, 273. Aktas, A., et al. (H1), 2005e, Eur.Phys.J. C38, 437. Aktas, A., et al. (H1), 2006a, Eur.Phys.J. C48, 749. Aktas, A., et al. (H1), 2006b, Phys.Lett. B638, 422. Aktas, A., et al. (H1), 2006c, Eur.Phys.J. C46, 585. Aktas, A., et al. (H1), 2006d, Eur.Phys.J. C46, 27. Aktas, A., et al. (H1), 2006e, Eur.Phys.J. C48, 715. Aktas, A., et al. (H1), 2006f, Eur.Phys.J. C47, 597. Aktas, A., et al. (H1), 2006g, Eur.Phys.J. C46, 343. Aktas, A., et al. (H1), 2006h, Phys.Lett. B639, 21. Aktas, A., et al. (H1), 2006i, Phys.Lett. B639, 202. Aktas, A., et al. (H1), 2007a, Eur.Phys.J. C50, 1. Aktas, A., et al. (H1), 2007b, JHEP 0710, 042.

Aktas, A., et al. (H1), 2007c, Eur.Phys.J. C50, 251. Aktas, A., et al. (H1), 2007d, Phys.Lett. B653, 134. Aktas, A., et al. (H1), 2007e, Eur.Phys.J. C52, 507.

Aktas, A., et al. (H1), 2007f, Eur.Phys.J. C51, 549.

Albaladejo, M., and J. Oller, 2008, Phys.Rev.Lett. 101, 252002.

Alberi, G., and G. Goggi, 1981, Phys.Rept. 74, 1.

Albino, S., B. A. Kniehl, and G. Kramer, 2005, Nucl.Phys. B725, 181.

Albino, S., B. A. Kniehl, and G. Kramer, 2008, Nucl.Phys. B803, 42 .

Albrecht, H., et al. (ARGUS), 1985, Phys.Lett. B157, 326.

Albrecht, H., et al. (ARGUS), 1990, Phys.Lett. B236, 102.

Alekhin, S., J. Blumlein, S. Klein, and S. Moch, 2010, Phys.Rev. D81, 014032.

Alekhin, S., J. Blumlein, and S. Moch, 2012, Phys.Rev. D86, 054009

Alekhin, S., and S. Moch, 2011, Phys.Lett. B699, 345.

Alexa, C., et al. (H1), 2013a, Eur.Phys.J. C73, 2466.

Alexa, C., et al. (H1), 2013b, Eur.Phys.J. C73, 2406.

Alexakhin, V. Y., et al. (COMPASS), 2007, Eur.Phys.J. C52, 255.

Alexander, G., 2003, Rept.Prog.Phys. 66, 481.

Alexander, G., I. Cohen, and E. Levin, 1999, Phys.Lett. B452, 159.

Alexopoulos, T., et al. (E735), 1993, Phys.Rev. D48, 1931. Allfrey, P. D., et al., 2007, Nucl.Instrum.Meth. A580, 1257.

Alper, B., H. Boeggild, P. Booth, F. Bulos, L. Carroll, et al., 1973, Phys.Lett. B46, 265.

Alt, C., et al. (NA49), 2004, Phys.Rev.Lett. 92, 042003.

Altarelli, G., and G. Parisi, 1977, Nucl.Phys. B126, 298.

Althoff, M., et al. (TASSO), 1983, Phys.Lett. B121, 216.

Althoff, M., et al. (TASSO), 1986, Z.Phys. C30, 355.

Amsler, C., et al. (Particle Data Group), 2008, Phys.Lett. B667, 1.

Andersson, B., G. Gustafson, G. Ingelman, and T. Sjostrand, 1983, Phys.Rept. 97, 31.

Andersson, B., G. Gustafson, L. Lonnblad, and U. Pettersson, 1989, Z.Phys. C43, 625.

Andresen, A., et al. (ZEUS Calorimeter Group), 1991, Nucl.Instrum.Meth. A309, 101.

Andrieu, B., et al. (H1 Calorimeter Group), 1993, Nucl.Instrum.Meth. A336, 460.

Anticic, T., et al. (NA49), 2012, Phys.Rev. C85, 044913.

Antipov, Y., S. Denisov, S. Donskov, Y. Gorin, V. Kachanov, et al., 1971, Phys.Lett. B34, 164. 
Aoki, M., J. Beatty, D. Beavis, J. Carroll, J. Chiba, et al., 1992, Phys.Rev.Lett. 69, 2345.

Appelquist, G., et al. (NA52 (NEWMASS)), 1996, Phys.Lett. B376, 245.

Appuhn, R. D., et al. (H1 SPACAL Group), 1997, Nucl.Instrum.Meth. A386, 397.

Arneodo, M., et al. (EMC), 1986, Z.Phys. C32, 1.

Arneodo, M., et al. (NMC), 1994, Nucl.Phys. B429, 503.

Arsene, I., et al. (BRAHMS), 2011, Phys.Rev. C83, 044906.

Artoisenet, P., J. M. Campbell, F. Maltoni, and F. Tramontano, 2009, Phys.Rev.Lett. 102, 142001.

Asner, D., et al. (CLEO), 2007, Phys.Rev. D75, 012009.

Aurenche, P., R. Baier, M. Fontannaz, M. Kienzle-Focacci, and M. Werlen, 1989, Phys.Lett. B233, 517.

Aurenche, P., L. Bourhis, M. Fontannaz, and J. P. Guillet, 2000, Eur.Phys.J. C17, 413.

Aurenche, P., M. Fontannaz, and J. P. Guillet, 2005, Eur.Phys.J. C44, 395.

Baier, R., and R. Ruckl, 1981, Phys.Lett. B102, 364.

Baier, R., and R. Ruckl, 1982, Nucl.Phys. B201, 1.

Baier, R., and R. Ruckl, 1983, Z.Phys. C19, 251.

Baird, A., et al. (H1), 2001, Nucl.Instrum.Meth. A461, 461.

Balitsky, I. I., and V. M. Braun, 1993, Phys.Lett. B314, 237.

Balitsky, I. I., and L. N. Lipatov, 1978, Sov.J.Nucl.Phys. 28, 822 .

Baranov, S., 1998, Phys.Lett. B428, 377.

Baranov, S. P., 2002, Phys.Rev. D66, 114003.

Bartel, W., et al. (JADE), 1986, Z.Phys. C33, 23.

Bartels, J., J. R. Ellis, H. Kowalski, and M. Wusthoff, 1999a, Eur.Phys.J. C7, 443.

Bartels, J., J. R. Forshaw, H. Lotter, and M. Wusthoff, 1996a, Phys.Lett. B375, 301.

Bartels, J., H. Jung, and A. Kyrieleis, 2002, Eur.Phys.J. C24, 555 .

Bartels, J., H. Jung, and M. Wusthoff, 1999b, Eur.Phys.J. C11, 111.

Bartels, J., H. Lotter, and M. Wusthoff, 1996b, Phys.Lett. B379, 239.

Bartsch, D., 2007, PhD Thesis BONN-IR-2007-05.

Bassler, U., and G. Bernardi, 1995, Nucl.Instrum.Meth. A361, 197.

Bauer, T., R. Spital, D. Yennie, and F. Pipkin, 1978, Rev.Mod.Phys. 50, 261.

Bearden, I., H. Boggild, J. Boissevain, P. Christiansen, L. Conin, et al., 2002, Eur.Phys.J. C23, 237.

Bearden, I., et al. (NA44), 1999, Nucl.Phys. A661, 387.

Belavin, A., A. M. Polyakov, A. Schwartz, and Y. Tyupkin, 1975, Phys.Lett. B59, 85.

Bentvelsen, S., J. Engelen, and P. Kooijman, 1992, in Hamburg, Proceedings, Physics at HERA, W. Buchmueller and G. Ingelman (eds.), Vol. 1, p.23.

Berger, E. L., J. C. Collins, D. E. Soper, and G. F. Sterman, 1987, Nucl. Phys. B286, 704.

Berger, E. L., and D. L. Jones, 1981, Phys.Rev. D23, 1521.

Beringer, J., et al. (Particle Data Group), 2012, Phys.Rev. D86, 010001.

Bernstein, A., et al. (ZEUS Barrel Calorimeter Group), 1993, Nucl.Instrum.Meth. A336, 23.

Bethke, S., 2004, Nucl.Phys.Proc.Suppl. 135, 345.

Bethke, S., et al. (JADE), 1988, Phys.Lett. B213, 235.

Bhadra, S., et al., 1995, Nucl.Instrum.Meth. A354, 479.

Bhadra, S., et al. (ZEUS FNC Group), 1997a, Nucl.Instrum.Meth. A394, 121.

Bhadra, S., et al., 1997b, given at 7th International Confer- ence on Calorimetry in High-Energy Physics (ICCHEP 97), Tucson, AZ, 9-14 Nov 1997.

Biddulph, P., H. Cronstrom, P. Finnegan, J. Foster, S. Gilbert, et al., 1994, Nucl.Instrum.Meth. A340, 304.

Binkley, M. E., C. Bohler, J. Butler, J. P. Cumalat, I. Gaines, et al., 1982, Phys.Rev.Lett. 48, 73.

Binnewies, J., B. A. Kniehl, and G. Kramer, 1995, Phys.Rev. D52, 4947.

Binnewies, J., B. A. Kniehl, and G. Kramer, 1997, Z.Phys. C76, 677.

Binnewies, J., B. A. Kniehl, and G. Kramer, 1998, Phys.Rev. D58, 014014.

Binon, F., et al. (IHEP-CERN Collaboration), 1969, Phys.Lett. B30, 510.

Bishari, M., 1972, Phys.Lett. B38, 510.

Bjorken, J., 1993, Phys.Rev. D47, 101.

Blok, B., L. Frankfurt, and M. Strikman, 2010, Phys.Lett. B690, 159.

Blumlein, J., and D. Robaschik, 2001, Phys.Lett. B517, 222.

Bodwin, G. T., E. Braaten, and G. P. Lepage, 1995, Phys.Rev. D51, 1125, erratum-ibid. 1997, D55, 5853.

Brambilla, N., S. Eidelman, B. Heltsley, R. Vogt, G. Bodwin, et al., 2011, Eur.Phys.J. C71, 1534.

Brandt, A., et al. (UA8), 1992, Phys.Lett. B297, 417.

Braunschweig, W., et al. (TASSO), 1990, Z.Phys. C47, 187.

Breitweg, J., et al. (ZEUS), 1997a, Z.Phys. C76, 599.

Breitweg, J., et al. (ZEUS), 1997b, Phys.Lett. B413, 201.

Breitweg, J., et al. (ZEUS), 1997c, Phys.Lett. B414, 428.

Breitweg, J., et al. (ZEUS), 1997d, Z.Phys. C75, 421.

Breitweg, J., et al. (ZEUS), 1998a, Eur.Phys.J. C2, 77.

Breitweg, J., et al. (ZEUS), 1998b, Eur.Phys.J. C1, 109.

Breitweg, J., et al. (ZEUS), 1998c, Eur.Phys.J. C2, 247.

Breitweg, J., et al. (ZEUS), 1998d, Phys.Lett. B421, 368.

Breitweg, J., et al. (ZEUS), 1998e, Eur.Phys.J. C2, 61.

Breitweg, J., et al. (ZEUS), 1999a, Eur.Phys.J. C6, 603.

Breitweg, J., et al. (ZEUS), 1999b, Eur.Phys.J. C6, 67.

Breitweg, J., et al. (ZEUS), 1999c, Eur.Phys.J. C8, 367.

Breitweg, J., et al. (ZEUS), 1999d, Eur.Phys.J. C11, 251.

Breitweg, J., et al. (ZEUS), 1999e, Eur.Phys.J. C6, 43.

Breitweg, J., et al. (ZEUS), 2000a, Eur.Phys.J. C14, 213.

Breitweg, J., et al. (ZEUS), 2000b, Phys.Lett. B472, 175.

Breitweg, J., et al. (ZEUS), 2000c, Eur.Phys.J. C12, 393.

Breitweg, J., et al. (ZEUS), 2001a, Phys.Lett. B507, 70.

Breitweg, J., et al. (ZEUS), 2001b, Eur.Phys.J. C18, 625.

Brock, R., et al. (CTEQ), 1995, Rev.Mod.Phys. 67, 157.

Brodsky, S. J., V. S. Fadin, V. T. Kim, L. N. Lipatov, and G. B. Pivovarov, 1999, JETP Lett. 70, 155.

Brodsky, S. J., L. Frankfurt, J. Gunion, A. H. Mueller, and M. Strikman, 1994, Phys.Rev. D50, 3134.

Buchmuller, W., T. Gehrmann, and A. Hebecker, 1999, Nucl.Phys. B537, 477.

Buchmuller, W., and A. Hebecker, 1995, Phys.Lett. B355, 573.

Buchmuller, W., M. McDermott, and A. Hebecker, 1997, Phys.Lett. B410, 304.

Burger, J., et al., 1989, Nucl.Instrum.Meth. A279, 217.

Butenschoen, M., and B. A. Kniehl, 2010, Phys.Rev.Lett. 104, 072001.

Butenschoen, M., and B. A. Kniehl, 2011a, Phys.Rev.Lett. 106, 022003.

Butenschoen, M., and B. A. Kniehl, 2011b, Phys.Rev. D84, 051501.

Butenschoen, M., and B. A. Kniehl, 2012, Nucl.Phys.Proc.Suppl. 222-224, 151. 
Butler, S., and C. Pearson, 1963, Phys.Rev. 129, 836.

Butterworth, J. M., A. R. Davison, M. Rubin, and G. P. Salam, 2008, Phys.Rev.Lett. 100, 242001.

Butterworth, J. M., J. R. Forshaw, and M. H. Seymour, 1996, Z.Phys. C72, 637.

Butterworth, J. M., and M. Wing, 2005, Rept.Prog.Phys. 68, 2773.

Cacciari, M., 2004, eprint hep-ph/0407187.

Cacciari, M., P. Nason, and C. Oleari, 2006, JHEP 0604, 006.

Cacciari, M., G. P. Salam, and G. Soyez, 2008, JHEP 0804, 063.

Caldwell, A., I. Gialas, S. Mishra, J. Parsons, S. Ritz, et al., 1992, Nucl.Instrum.Meth. A321, 356.

Camacho, C. M., et al. (Jefferson Lab Hall A), 2006, Phys.Rev.Lett. 97, 262002.

Caron, S., 2002, PhD Thesis DESY-THESIS-2002-035.

Caswell, W., and G. Lepage, 1986, Phys.Lett. B167, 437.

Catani, S., Y. L. Dokshitzer, M. H. Seymour, and B. R. Webber, 1993, Nucl.Phys. B406, 187.

Catani, S., F. Fiorani, and G. Marchesini, 1990a, Phys.Lett. B234, 339.

Catani, S., F. Fiorani, and G. Marchesini, 1990b, Nucl.Phys. B336, 18.

Catani, S., and M. H. Seymour, 1997, Nucl.Phys. B485, 291, erratum-ibid. 1998, B510, 503.

Catani, S., B. R. Webber, Y. L. Dokshitzer, and F. Fiorani, 1992, Nucl.Phys. B383, 419.

Chang, C.-H., 1980, Nucl.Phys. B172, 425.

Chapin, T., R. Cool, K. A. Goulianos, K. Jenkins, J. Silverman, et al., 1985, Phys.Rev. D31, 17.

Chatrchyan, S., et al. (CMS), 2011, JHEP 1109, 109.

Chatrchyan, S., et al. (CMS), 2012a, JHEP 1202, 011.

Chatrchyan, S., et al. (CMS), 2012b, JHEP 1208, 130.

Chatrchyan, S., et al. (CMS), 2013a, Phys.Rev. D87, 012006.

Chatrchyan, S., et al. (CMS), 2013b, JHEP 1304, 072.

Chekanov, S., et al. (ZEUS), 2001a, Phys.Lett. B510, 36.

Chekanov, S., et al. (ZEUS), 2001b, Phys.Lett. B511, 19.

Chekanov, S., et al. (ZEUS), 2001c, Phys.Lett. B516, 273.

Chekanov, S., et al. (ZEUS), 2002a, Eur.Phys.J. C23, 615.

Chekanov, S., et al. (ZEUS), 2002b, Eur.Phys.J. C23, 13.

Chekanov, S., et al. (ZEUS), 2002c, Eur.Phys.J. C24, 345.

Chekanov, S., et al. (ZEUS), 2002d, Phys.Lett. B531, 9.

Chekanov, S., et al. (ZEUS), 2002e, Phys.Lett. B547, 164.

Chekanov, S., et al. (ZEUS), 2002f, Phys.Rev. D65, 052001.

Chekanov, S., et al. (ZEUS), 2003a, Eur.Phys.J. C27, 531.

Chekanov, S., et al. (ZEUS), 2003b, Eur.Phys.J. C26, 389.

Chekanov, S., et al. (ZEUS), 2003c, Phys.Lett. B558, 41.

Chekanov, S., et al. (ZEUS), 2003d, Nucl.Phys. B672, 3.

Chekanov, S., et al. (ZEUS), 2003e, Eur.Phys.J. C27, 173.

Chekanov, S., et al. (ZEUS), 2003f, Phys.Lett. B551, 226.

Chekanov, S., et al. (ZEUS), 2004a, Phys.Rev. D70, 012008, erratum-ibid. 2006, D74, 059906.

Chekanov, S., et al. (ZEUS), 2004b, Phys.Lett. B583, 231.

Chekanov, S., et al. (ZEUS), 2004c, Phys.Lett. B591, 7.

Chekanov, S., et al. (ZEUS), 2004d, Nucl.Phys. B695, 3.

Chekanov, S., et al. (ZEUS), 2004e, Phys.Lett. B595, 86.

Chekanov, S., et al. (ZEUS), 2004f, Phys.Lett. B578, 33.

Chekanov, S., et al. (ZEUS), 2004g, Phys.Lett. B590, 143.

Chekanov, S., et al. (ZEUS), 2004h, Eur.Phys.J. C38, 29.

Chekanov, S., et al. (ZEUS), 2004i, Eur.Phys.J. C34, 255.

Chekanov, S., et al. (ZEUS), 2004j, Nucl.Phys. B700, 3.

Chekanov, S., et al. (ZEUS), 2005a, Eur.Phys.J. C42, 1.

Chekanov, S., et al. (ZEUS), 2005b, Nucl.Phys. B729, 492.

Chekanov, S., et al. (ZEUS), 2005c, Eur.Phys.J. C44, 351.
Chekanov, S., et al. (ZEUS), 2005d, Eur.Phys.J. C44, 13.

Chekanov, S., et al. (ZEUS), 2005e, Eur.Phys.J. C44, 183. Chekanov, S., et al. (ZEUS), 2005f, Phys.Lett. B610, 212. Chekanov, S., et al. (ZEUS), 2005g, Nucl.Phys. B713, 3. Chekanov, S., et al. (ZEUS), 2005h, Phys.Lett. B610, 199. Chekanov, S., et al. (ZEUS), 2007a, Phys.Lett. B652, 1. Chekanov, S., et al. (ZEUS), 2007b, Eur.Phys.J. C51, 301. Chekanov, S., et al. (ZEUS), 2007c, Eur.Phys.J. C52, 813. Chekanov, S., et al. (ZEUS), 2007d, Nucl.Phys. B767, 1. Chekanov, S., et al. (ZEUS), 2007e, PMC Phys. A1, 6. Chekanov, S., et al. (ZEUS), 2007f, Phys.Rev. D76, 072011. Chekanov, S., et al. (ZEUS), 2007g, Nucl.Phys. B765, 1. Chekanov, S., et al. (ZEUS), 2007h, Phys.Lett. B649, 12. Chekanov, S., et al. (ZEUS), 2007i, Nucl.Phys. B776, 1. Chekanov, S., et al. (ZEUS), 2007j, Nucl.Phys. B786, 181. Chekanov, S., et al. (ZEUS), 2007k, JHEP 0707, 074. Chekanov, S., et al. (ZEUS), 2007l, Eur.Phys.J. C50, 299. Chekanov, S., et al. (ZEUS), 2007m, Eur.Phys.J. C49, 511. Chekanov, S., et al. (ZEUS), 2007n, Eur.Phys.J. C50, 283. Chekanov, S., et al. (ZEUS), 2008a, Phys.Rev. D78, 072001. Chekanov, S., et al. (ZEUS), 2008b, Nucl.Phys. B800, 1. Chekanov, S., et al. (ZEUS), 2008c, Eur.Phys.J. C55, 177. Chekanov, S., et al. (ZEUS), 2008d, JHEP 0806, 061. Chekanov, S., et al. (ZEUS), 2008e, Phys.Rev.Lett. 101, 112003.

Chekanov, S., et al. (ZEUS), 2008f, Nucl.Phys. B792, 1. Chekanov, S., et al. (ZEUS), 2009a, JHEP 0905, 108. Chekanov, S., et al. (ZEUS), 2009b, Nucl.Phys. B816, 1. Chekanov, S., et al. (ZEUS), 2009c, JHEP 0906, 074. Chekanov, S., et al. (ZEUS), 2009d, JHEP 0904, 133. Chekanov, S., et al. (ZEUS), 2009e, JHEP 0902, 032. Chekanov, S., et al. (ZEUS), 2009f, JHEP 0912, 007. Chekanov, S., et al. (ZEUS), 2009g, JHEP 0904, 082. Chekanov, S., et al. (ZEUS), 2009h, Phys.Lett. B682, 8. Chekanov, S., et al. (ZEUS), 2009i, Eur.Phys.J. C60, 25. Chekanov, S., et al. (ZEUS), 2009j, Eur.Phys.J. C60, 25. Chekanov, S., et al. (ZEUS), 2009k, Eur.Phys.J. C63, 527. Chekanov, S., et al. (ZEUS), 2010a, Nucl.Phys. B831, 1. Chekanov, S., et al. (ZEUS), 2010b, Eur.Phys.J. C65, 65. Chekanov, S., et al. (ZEUS), 2010c, Nucl.Phys. B827, 1. Chekanov, S., et al. (ZEUS), 2010d, Phys.Lett. B687, 16. Chekanov, S., et al. (ZEUS), 2010e, Phys.Lett. B687, 16. Chekanov, S., et al. (ZEUS), 2010f, JHEP 1005, 085.

Chekanov, S., et al. (ZEUS), 2012, Phys.Rev. D85, 052008.

Chew, G. F., and S. C. Frautschi, 1961, Phys.Rev.Lett. 7, 394.

Chew, G. F., S. C. Frautschi, and S. Mandelstam, 1961, Phys.Rev. 126, 1202.

Cho, P. L., and A. K. Leibovich, 1996a, Phys.Rev. D53, 150. Cho, P. L., and A. K. Leibovich, 1996b, Phys.Rev. D53, 6203. Choi, S., et al. (AMY), 1995, Phys.Lett. B355, 406.

Chyla, J., J. Cvach, K. Sedlak, and M. Tasevsky, 2005, Eur.Phys.J. C40, 469.

Ciafaloni, M., 1988, Nucl.Phys. B296, 49.

Collins, J. C., 1998, Phys.Rev. D57, 3051, erratum-ibid. 2000, D61, 019902.

Collins, J. C., L. Frankfurt, and M. Strikman, 1997, Phys.Rev. D56, 2982.

Corcella, G., et al., 2001, JHEP 01, 010.

Cornet, F., P. Jankowski, and M. Krawczyk, 2004, Phys.Rev. D70, 093004.

Cox, B. E., and J. R. Forshaw, 2002, Comput.Phys.Commun. 144, 104.

Cronin, J., H. J. Frisch, M. Shochet, J. Boymond, R. Mermod, 
et al., 1975, Phys.Rev. D11, 3105.

Cudell, J. R., K. Kang, and S. K. Kim, 1997, Phys.Lett. B395, 311.

Danilov, M., and R. Mizuk, 2008, Phys.Atom.Nucl. 71, 605.

Dederichs, K. H., and M. A. Faessler, 1989, Phys. Lett. B232, 405.

Denby, B. H., V. Bharadwaj, D. Summers, A. Eisner, R. Kennett, et al., 1984, Phys.Rev.Lett. 52, 795.

Derrick, M., D. Gacek, N. Hill, B. Musgrave, R. Noland, et al., 1991, Nucl.Instrum.Meth. A309, 77.

Derrick, M., et al. (ZEUS), 1992, Phys.Lett. B297, 404.

Derrick, M., et al. (ZEUS), 1993, Phys.Lett. B315, 481.

Derrick, M., et al. (ZEUS), 1994a, Phys.Lett. B322, 287.

Derrick, M., et al. (ZEUS), 1994b, Phys.Lett. B332, 228.

Derrick, M., et al. (ZEUS), 1995a, Phys.Lett. B348, 665.

Derrick, M., et al. (ZEUS.), 1995b, Z.Phys. C67, 227.

Derrick, M., et al. (ZEUS), 1995c, Z.Phys. C67, 93.

Derrick, M., et al. (ZEUS), 1995d, Phys.Lett. B346, 399.

Derrick, M., et al. (ZEUS), 1996a, Z.Phys. C70, 1.

Derrick, M., et al. (ZEUS), 1996b, Phys.Lett. B369, 55.

Derrick, M., et al. (ZEUS), 1997, Z.Phys. C73, 253.

Devenish, R., and A. Cooper-Sarkar, 2004, Deep inelastic scattering OUP, $403 \mathrm{p}$.

Diakonov, D., V. Petrov, and M. V. Polyakov, 1997, Z.Phys. A359, 305.

Diehl, M., 2003, Phys.Rept. 388, 41.

Dissertori, G., I. Knowles, and M. Schmelling, 2003, International Series of Monographs on Physics, Oxford Univ. Press $115,1$.

Dokshitzer, Y. L., 1977, Sov.Phys.JETP 46, 641.

Dokshitzer, Y. L., V. A. Khoze, and T. Sjostrand, 1992, Phys.Lett. B274, 116.

Dokshitzer, Y. L., A. Lucenti, G. Marchesini, and G. Salam, 1998, Nucl.Phys. B511, 396, erratum-ibid. 2001, B593, 729.

Dokshitzer, Y. L., G. Marchesini, and G. Salam, 1999, Eur.Phys.J.direct C1, 3.

Dokshitzer, Y. L., G. Marchesini, and B. Webber, 1996, Nucl.Phys. B469, 93.

Dokshitzer, Y. L., and B. Webber, 1995, Phys.Lett. B352, 451.

Dokshitzer, Y. L., and B. Webber, 1997, Phys.Lett. B404, 321.

Donnachie, A., and P. Landshoff, 1984, Nucl.Phys. B244, 322 .

Donnachie, A., and P. Landshoff, 1992, Phys.Lett. B296, 227.

Donnachie, A., and P. Landshoff, 1998, Phys.Lett. B437, 408.

Donnachie, A., and P. V. Landshoff, 1987, Phys.Lett. B191, 309, erratum-ibid. 1987, B198, 590.

Dorfan, D., J. Eades, L. Lederman, W. Lee, and C. Ting, 1965, Phys.Rev.Lett. 14, 1003.

Dzierba, A. R., C. A. Meyer, and A. P. Szczepaniak, 2005, J.Phys.Conf.Ser. 9, 192.

Edin, A., G. Ingelman, and J. Rathsman, 1996, Phys.Lett. B366, 371.

Eick, W., H. Henschel, H. Kaufmann, M. Klein, P. Kostka, et al., 1997, Nucl.Instrum.Meth. A386, 81.

Ellis, R. K., W. J. Stirling, and B. Webber, 1996, Camb.Monogr.Part.Phys.Nucl.Phys.Cosmol. 8, 1.

Ellis, S. D., Z. Kunszt, and D. E. Soper, 1992, Phys.Rev.Lett. 69, 3615 .

Ellis, S. D., and D. E. Soper, 1993, Phys.Rev. D48, 3160.

Enberg, R., J. R. Forshaw, L. Motyka, and G. Poludniowski, 2003, JHEP 0309, 008.
Enberg, R., G. Ingelman, and L. Motyka, 2002a, Phys.Lett. B524, 273.

Enberg, R., L. Motyka, and G. Poludniowski, 2002b, Eur.Phys.J. C26, 219.

Faiman, D., H. Lipkin, and H. Rubinstein, 1975, Phys.Lett. B59, 269.

Feinberg, E., and I. Pomerančuk, 1956, Suppl.Nuovo Cim. 3, 652.

Feynman, R. P., 1972, Benjamin, New York, Photon-Hadron Interactions.

de Florian, D., R. Sassot, and M. Stratmann, 2007a, Phys.Rev. D75, 114010.

de Florian, D., R. Sassot, and M. Stratmann, 2007b, Phys.Rev. D76, 074033.

Fontannaz, M., J. P. Guillet, and G. Heinrich, 2001, Eur.Phys.J. C21, 303.

Fontannaz, M., J. P. Guillet, and G. Heinrich, 2002, Eur.Phys.J. C26, 209.

Fontannaz, M., and G. Heinrich, 2004, Eur.Phys.J. C34, 191.

Forshaw, J. R., G. Kerley, and G. Shaw, 1999, Phys.Rev. D60, 074012.

Forshaw, J. R., and G. Poludniowski, 2003, Eur.Phys.J. C26, 411.

Forshaw, J. R., and M. Ryskin, 1995, Z.Phys. C68, 137.

Forshaw, J. R., R. Sandapen, and G. Shaw, 2004, Phys.Rev. D69, 094013.

Forshaw, J. R., and M. H. Seymour, 1999, JHEP 09, 009.

Forshaw, J. R., and P. Sutton, 1998, Eur.Phys.J. C1, 285.

Foster, B., et al., 1993, Nucl.Phys.Proc.Suppl. 32, 181.

Foster, B., et al., 1994, Nucl.Instrum.Meth. A338, 254.

Frankfurt, L., A. Freund, and M. Strikman, 1998, Phys.Rev. D58, 114001.

Frankfurt, L., V. Guzey, M. McDermott, and M. Strikman, 2001a, eprint hep-ph/0104252.

Frankfurt, L., W. Koepf, and M. Strikman, 1996, Phys.Rev. D54, 3194.

Frankfurt, L., W. Koepf, and M. Strikman, 1997, Phys.Lett. B405, 367.

Frankfurt, L., M. McDermott, and M. Strikman, 2001b, JHEP 0103, 045.

Frankfurt, L., M. Strikman, and M. Zhalov, 2008, Phys.Lett. B670, 32.

Frixione, S., 1997, Nucl.Phys. B507, 295.

Frixione, S., Z. Kunszt, and A. Signer, 1996, Nucl.Phys. B467, 399.

Frixione, S., M. L. Mangano, P. Nason, and G. Ridolfi, 1993, Phys.Lett. B319, 339.

Frixione, S., M. L. Mangano, P. Nason, and G. Ridolfi, 1995a, Phys.Lett. B348, 633.

Frixione, S., P. Nason, and G. Ridolfi, 1995b, Nucl.Phys. B454, 3 .

Frixione, S., and G. Ridolfi, 1997, Nucl.Phys. B507, 315.

Frixione, S., and B. R. Webber, 2002, JHEP 0206, 029.

Gavalian, G., et al. (CLAS), 2009, Phys.Rev. C80, 035206.

Gehrmann-De Ridder, A., T. Gehrmann, and E. Poulsen, 2006a, Phys.Rev.Lett. 96, 132002.

Gehrmann-De Ridder, A., T. Gehrmann, and E. Poulsen, 2006b, Eur.Phys.J. C47, 395.

Gehrmann-De Ridder, A., G. Kramer, and H. Spiesberger, 2000, Nucl.Phys. B578, 326.

Gibbs, M., A. Ringwald, and F. Schrempp, 1995, eprint hepph/9506392.

Ginzburg, I., S. Panfil, and V. Serbo, 1987, Nucl.Phys. B284, 685. 
Girod, F., et al. (CLAS), 2008, Phys.Rev.Lett. 100, 162002. Gladilin, L., 1999, eprint hep-ex/9912064.

Glazov, A. A., 1998, PhD Thesis DESY-THESIS-1998-005.

Gluck, M., E. Reya, and I. Schienbein, 1999, Eur.Phys.J. C10, 313.

Gluck, M., E. Reya, and M. Stratmann, 1996, Phys.Rev. D54, 5515 .

Goldhaber, G., W. B. Fowler, S. Goldhaber, and T. Hoang, 1959, Phys.Rev.Lett. 3, 181.

Goldhaber, G., S. Goldhaber, W.-Y. Lee, and A. Pais, 1960, Phys.Rev. 120, 300.

Golec-Biernat, K. J., and A. Luszczak, 2007, Phys.Rev. D76, 114014.

Golec-Biernat, K. J., and M. Wusthoff, 1998, Phys.Rev. D59, 014017.

Golec-Biernat, K. J., and M. Wusthoff, 1999, Phys.Rev. D60, 114023.

Good, M. L., and W. D. Walker, 1960, Phys.Rev. 120, 1857.

Gordon, L. E., and J. K. Storrow, 1992, Phys.Lett. B291, 320 .

Gordon, L. E., and J. K. Storrow, 1994, Z.Phys. C63, 581.

Gotsman, E., A. Kormilitzin, E. Levin, and U. Maor, 2007, Eur.Phys.J. C52, 295.

Gotsman, E., E. Levin, U. Maor, and E. Naftali, 2002, Phys.Lett. B532, 37.

Goulianos, K. A., 1983, Phys.Rept. 101, 169.

Graudenz, D., 1997, eprint hep-ph/9710244.

Gribov, L. V., E. M. Levin, and M. G. Ryskin, 1983, Phys.Rept. 100, 1.

Gribov, V., 1970, Sov.Phys.JETP 30, 709.

Gribov, V. N., and L. N. Lipatov, 1972, Sov.J.Nucl.Phys. 15, 438.

Gross, D., and F. Wilczek, 1973a, Phys.Rev. D8, 3633.

Gross, D., and F. Wilczek, 1973b, Phys.Rev.Lett. 30, 1343.

H1, 1992-, H1 publications: http://h1.desy.de/e104552/e104555/.

Harnew, N., et al., 1989, Nucl.Instrum.Meth. A279, 290.

Harris, B. W., and J. F. Owens, 1997, Phys.Rev. D56, 4007.

Harris, B. W., and J. Smith, 1995a, Nucl.Phys. B452, 109.

Harris, B. W., and J. Smith, 1995b, Phys.Lett. B353, 535.

Harris, B. W., and J. Smith, 1998, Phys.Rev. D57, 2806.

Hebecker, A., and T. Teubner, 2001, Phys.Lett. B498, 16.

Henning, S., et al. (British-Scandinavian-MIT Collaboration), 1978, Lett.Nuovo Cim. 21, 189.

Hicks, K., 2005a, J.Phys.Conf.Ser. 9, 183.

Hicks, K. H., 2005b, Prog.Part.Nucl.Phys. 55, 647.

Hicks, K. H., 2012, Eur.Phys.J. H37, 1.

Hoeger, K. C., 1992, in Hamburg, Proceedings, Physics at HERA, W. Buchmueller and G. Ingelman (eds.), Vol. 1, p.43.

Holtmann, H., G. Levman, N. N. Nikolaev, A. Szczurek, and J. Speth, 1994, Phys.Lett. B338, 363.

't Hooft, G., 1976a, Phys.Rev. D14, 3432, erratum-ibid. 1978, D18, 2199.

't Hooft, G., 1976b, Phys.Rev.Lett. 37, 8.

Iancu, E., K. Itakura, and S. Munier, 2004, Phys.Lett. B590, 199.

Ingelman, G., and P. E. Schlein, 1985, Phys.Lett. B152, 256.

Ivanov, I., N. Nikolaev, and A. Savin, 2006, Phys.Part.Nucl. 37,1 .

Jacquet, F., and A. Blondel, 1979, in Hamburg, Proceedings, Study Of An E P Facility For Europe, U. Amaldi (ed.), p.391.

Jaffe, R. L., 1977, Phys.Rev. D15, 281.
Jimenez-Delgado, P., and E. Reya, 2009, Phys.Rev. D79, 074023.

Jung, H., 1995, Comput.Phys.Commun. 86, 147.

Jung, H., 2002, Comput.Phys.Commun. 143, 100.

Jung, H., and G. P. Salam, 2001, Eur.Phys.J. C19, 351.

Juricic, I., G. Goldhaber, G. Gidal, G. Abrams, D. Amidei, et al., 1989, Phys.Rev. D39, 1.

Kaidalov, A., V. Khoze, A. Martin, and M. Ryskin, 2003, Phys.Lett. B567, 61.

Kaidalov, A., V. Khoze, A. Martin, and M. Ryskin, 2006, Eur.Phys.J. C47, 385.

Kaidalov, A., V. Khoze, A. Martin, and M. Ryskin, 2010, Eur.Phys.J. C66, 373.

Kaidalov, A. B., 1979, Phys.Rept. 50, 157.

Kartvelishvili, V., A. Likhoded, and V. Petrov, 1978, Phys.Lett. B78, 615.

Khachatryan, V., et al. (CMS), 2010, Eur.Phys.J. C70, 555.

Khachatryan, V., et al. (CMS), 2011, JHEP 1105, 029.

Khoze, V., A. Martin, and M. Ryskin, 2006, Eur.Phys.J. C48, 797.

Klasen, M., and G. Kramer, 1997, Z.Phys. C76, 67.

Klasen, M., and G. Kramer, 2009, Phys.Rev. D80, 074006.

Klein, M., and R. Yoshida, 2008, Prog.Part.Nucl.Phys. 61, 343.

Klempt, E., and A. Zaitsev, 2007, Phys.Rept. 454, 1.

Kniehl, B. A., G. Kramer, and B. Potter, 2000, Phys.Rev.Lett. 85, 5288.

Kniehl, B. A., G. Kramer, and M. Spira, 1997, Z.Phys. C76, 689.

Korotkov, V., et al. (BBCNC), 1993, Z.Phys. C60, 37.

Kowalski, H., L. Motyka, and G. Watt, 2006, Phys.Rev. D74, 074016.

Kowalski, H., and D. Teaney, 2003, Phys.Rev. D68, 114005.

Kramer, M., 1996, Nucl.Phys. B459, 3.

Krawczyk, M., and A. Zembrzuski, 2001, Phys.Rev. D64, 114017.

Kretzer, S., 2000, Phys.Rev. D62, 054001.

Krueger, K. (H1), 2009, PoS EPS-HEP2009, 079.

Kumericki, K., and D. Mueller, 2010, Nucl.Phys. B841, 1.

Kuraev, E. A., L. N. Lipatov, and V. S. Fadin, 1976, Sov.Phys.JETP 44, 443.

Kuraev, E. A., L. N. Lipatov, and V. S. Fadin, 1977, Sov.Phys.JETP 45, 199.

Landshoff, P., and J. Polkinghorne, 1978, Phys.Rev. D18, 3344.

Li, Y., et al. (AMY), 1990, Phys.Rev. D41, 2675.

Lipatov, A., and N. Zotov, 2003, Eur.Phys.J. C27, 87.

Lipatov, A. V., and N. P. Zotov, 2005, Phys.Rev. D72, 054002.

Lipatov, L. N., 1975, Sov.J.Nucl.Phys. 20, 94.

Lipkin, H. J., 1987, Phys.Lett. B195, 484.

Lohrmann, E., 2011, eprint 1112.3757.

Lonnblad, L., 1995, Z.Phys. C65, 285.

Lotter, H., 1997, Phys.Lett. B406, 171.

Low, F., 1975, Phys.Rev. D12, 163.

Lukaszuk, L., and B. Nicolescu, 1973, Lett.Nuovo Cim. 8, 405.

Marchesini, G., 1995, Nucl.Phys. B445, 49.

Marchesini, G., et al., 1992, Comput.Phys.Commun. 67, 465.

Marquet, C., R. B. Peschanski, and G. Soyez, 2007, Phys.Rev. D76, 034011.

Martin, A., C. Nockles, M. G. Ryskin, and T. Teubner, 2008, Phys.Lett. B662, 252.

Martin, A., M. Ryskin, and G. Watt, 2007, Phys.Lett. B644, 
131.

Martin, A. D., R. G. Roberts, W. J. Stirling, and R. S. Thorne, 2005, Eur.Phys.J. C39, 155.

Martin, A. D., M. Ryskin, and T. Teubner, 1997, Phys.Rev. D55, 4329.

Martin, A. D., M. Ryskin, and T. Teubner, 2000, Phys.Rev. D62, 014022.

Martin, A. D., W. J. Stirling, R. S. Thorne, and G. Watt, 2009, Eur.Phys.J. C63, 189.

Massam, T., T. Muller, B. Righini, M. Schneegans, and A. Zichichi, 1965, Il Nuovo Cim. 39, 10.

Miettinen, H. I., and J. Pumplin, 1978, Phys.Rev. D18, 1696. Mirkes, E., and D. Zeppenfeld, 1996, Phys.Lett. B380, 205.

Moch, S., A. Ringwald, and F. Schrempp, 1997, Nucl.Phys. B507, 134.

Mueller, A. H., 1970, Phys.Rev. D2, 2963.

Mueller, A. H., 1990, Nucl.Phys. B335, 115.

Mueller, A. H., and W.-K. Tang, 1992, Phys.Lett. B284, 123.

Nagy, Z., and Z. Trocsanyi, 2001, Phys.Rev.Lett. 87, 082001.

Nason, P., 2004, JHEP 0411, 040.

Navin, S., 2010, eprint 1005.3894.

Newman, P., and M. Ruspa, 2009, eprint 0903.2957.

Newman, P. R., 2005, eprint hep-ex/0511047.

Nicholls, T., et al., 1998, IEEE Trans.Nucl.Sci. 45, 810.

Nikolaev, N., and B. G. Zakharov, 1992, Z.Phys. C53, 331.

Nikolaev, N. N., and B. Zakharov, 1991, Z.Phys. C49, 607.

Nikolaev, N. N., B. Zakharov, and V. Zoller, 1996, Phys.Lett.

B366, 337.

Nisius, R., 2000, Phys.Rept. 332, 165.

Nussinov, S., 1975, Phys.Rev.Lett. 34, 1286.

Okorokov, V., 2012, Int.J.Mod.Phys. A27, 1250037.

Perez, E., and E. Rizvi, 2013, Rep.Prog.Phys. 76, 046201.

Petersen, A., G. Abrams, C. Adolphsen, C. Akerlof, J. P. Alexander, et al., 1988, Phys.Rev. D37, 1.

Peterson, C., D. Schlatter, I. Schmitt, and P. M. Zerwas, 1983, Phys.Rev. D27, 105.

Pitzl, D., O. Behnke, M. Biddulph, K. Bosiger, R. Eichler, et al., 2000, Nucl.Instrum.Meth. A454, 334.

Polini, A., et al., 2007, Nucl.Instrum.Meth. A581, 656.

Politzer, H. D., 1973, Phys.Rev.Lett. 30, 1346.

Politzer, H. D., 1974, Phys.Rept. 14, 129.

Poludniowski, G., R. Enberg, J. R. Forshaw, and L. Motyka, 2003, JHEP 0312, 002.

Potter, B., 1999, Comput.Phys.Commun. 119, 45.

Rathsman, J., 1999, Phys.Lett. B452, 364.

Regge, T., 1959, Il Nuovo Cim. 14, 951.

Regge, T., 1960, Il Nuovo Cim. 18, 947.

Rezaeian, A. H., M. Siddikov, M. Van de Klundert, and R. Venugopalan, 2013, Phys.Rev. D87(3), 034002.

Ringwald, A., and F. Schrempp, 1994, eprint hep-ph/9411217. Ringwald, A., and F. Schrempp, 1998, Phys.Lett. B438, 217. Ringwald, A., and F. Schrempp, 1999, Phys.Lett. B459, 249. Ringwald, A., and F. Schrempp, 2000, Com-
put.Phys.Commun. 132, 267.

Ringwald, A., and F. Schrempp, 2001, Phys.Lett. B503, 331.

Royon, C., L. Schoeffel, J. Bartels, H. Jung, and R. B. Peschanski, 2001, Phys.Rev. D63, 074004.

Ryskin, M., 1993, Z.Phys. C57, 89.

Ryskin, M., A. Martin, and V. Khoze, 2011, Eur.Phys.J. C71, 1617.

Salam, G. P., and G. Soyez, 2007, JHEP 05, 086.

Schael, S., et al. (ALEPH), 2006, Phys.Lett. B639, 192.

Schilling, K., and G. Wolf, 1973, Nucl.Phys. B61, 381.

Sefkow, F., E. Elsen, H. Krehbiel, U. Straumann, and J. Coughlan, 1995, IEEE Trans.Nucl.Sci. 42, 900.

Seymour, M. H., 1994, Nucl.Phys. B421, 545.

Seymour, M. H., 1996, Phys.Lett. B378, 279.

Shambroom, W., R. Wilson, B. Gordon, W. Loomis, F. Pipkin, et al., 1982, Phys.Rev. D26, 1.

Sjostrand, T., 1994, Comput.Phys.Commun. 82, 74.

Sjostrand, T., S. Mrenna, and P. Skands, 2006, JHEP 0605, 026.

Sjostrand, T., et al., 2001, Comput.Phys.Commun. 135, 238.

Slominski, W., H. Abramowicz, and A. Levy, 2006, Eur.Phys.J. C45, 633.

Smith, W. H., K. Tokushuku, and L. W. Wiggers, 1992, contributed to 10th International Conference on Computing in High Energy Physics (CHEP 92), Annecy, France, 21-25 Sept 1992.

Steinhart, J., 1999, PhD Thesis DESY-THESIS-1999-029.

Streng, K. H., T. F. Walsh, and P. M. Zerwas, 1979, Z.Phys. C2, 237.

Strottman, D., 1979, Phys.Rev. D20, 748.

Szczurek, A., N. N. Nikolaev, and J. Speth, 1998, Phys.Lett. B428, 383.

Thacker, B., and G. P. Lepage, 1991, Phys.Rev. D43, 196.

Toll, T., 2010, PhD Thesis DESY-THESIS-2010-004.

Trentadue, L., and G. Veneziano, 1994, Phys.Lett. B323, 201.

Uematsu, T., and T. Walsh, 1981, Phys.Lett. B101, 263.

Van Esch, P., et al., 2000, Nucl.Instrum.Meth. A446, 409.

Voss, G., and B. Wiik, 1994, Ann.Rev.Nucl.Part.Sci. 44, 413.

von Weizsacker, C. F., 1934, Z.Phys. 88, 612.

Williams, E. J., 1934, Phys.Rev. 45, 729.

Wing, M. (ZEUS), 2002, eprint hep-ex/0206036.

Wolf, G., 2010, Rept.Prog.Phys. 73, 116202.

Wusthoff, M., 1997, Phys.Rev. D56, 4311.

Yao, W., et al. (Particle Data Group), 2006, J.Phys. G33, 1.

Zembrzuski, A., and M. Krawczyk, 2003, eprint hepph/0309308.

ZEUS, 1992-, ZEUS publications: http://www-zeus.desy.de/zeus_papers/zeus_papers.html.

ZEUS, 1993, U. Holm (ed.), The ZEUS Detector, Status Report (unpublished), DESY, available on http://www-zeus.desy.de/bluebook/bluebook.html.

Zotov, N., and V. Tsarev, 1988, Sov.Phys.Usp. 31, 119. 